\title{
EFFECTS OF OVARIECTOMY AND ANATOMICAL LOCATION ON OSTEONAL ENCROACHMENT IN ADULT CORTICAL OVINE BONE
}

\author{
A Thesis \\ Presented to \\ the Faculty of California Polytechnic State University, \\ San Luis Obispo
}

\author{
In Partial Fulfillment \\ of the Requirements for the Degree \\ Master of Science in Biomedical Engineering
}

By

Paige Ryan

February 2013 
(C) 2013

Paige Ryan

\section{ALL RIGHTS RESERVED}




\title{
COMMITTEE MEMBERSHIP
}

TITLE: $\quad$ EFFECTS OF OVARIECTOMY AND ANATOMICAL LOCATION ON OSTEONAL ENCROACHMENT IN ADULT CORTICAL OVINE BONE

\author{
AUTHOR: $\quad$ Paige Ryan \\ DATE SUBMITTED: $\quad$ February 2013 \\ COMMITTE CHAIR: $\quad$ Dr. Scott Hazelwood, Associate Professor \\ Biomedical and General Engineering Department \\ California Polytechnic State University, San Luis Obispo, CA \\ COMMITTEE MEMBER: Dr. Lanny Griffin, Department Chair \\ Biomedical and General Engineering Department \\ California Polytechnic State University, San Luis Obispo, CA \\ COMMITTEE MEMBER: Dr. Clifford Les, Associate Staff Scientist \\ Bone and Joint Center, \\ Henry Ford Hospital, Detroit, MI
}




\begin{abstract}
EFFECTS OF OVARIECTOMY AND ANATOMICAL LOCATION ON OSTEONAL ENCROACHMENT IN ADULT CORTICAL OVINE BONE
\end{abstract}

\author{
Paige Ryan
}

The purpose of this study is to further quantify adult ovine ovariectomized bone for new remodeling characteristics to obtain a better understanding of how remodeling is occurring and the effectiveness of this animal model for the study of postmenopausal osteoporosis.

Postmenopausal osteoporosis is a major health concern and animal models to test new treatment options are needed. The ovine model is a good option because the ewes undergo Haversian remodeling, are a large sized animal, and have a similar hormone profile to humans. Ewes, however, do not undergo a natural menopause, so an ovariectomy surgery was conducted in the sheep to simulate the decreased levels in estrogen. Columbia-Rambouillet sheep were used in this study: some that have been ovariectomized as a model for postmenopausal osteoporosis and some that underwent a sham surgery to serve as a control. The sheep were sacrificed 12 months post operatively in the month of August, so the seasonal effects of remodeling were accounted for. The left radius was then processed into microradiographs of 6 regional cortical beams, where the cranial (tensile side) and caudal (compressive side) anatomical sections were analyzed in this study to determine regional differences in remodeling. Previous students' theses have analyzed the similar samples for basic bone remodeling histology measurements, resulting in some significant seasonal, anatomical, and treatment differences. However, most of the results showed no particular increase in the amount of remodeled area for the ovariectomized sheep compared to the sham sheep, even though an ovariectomy is believed to cause a burst of remodeling in bone due to the decreased levels in estrogen. 
In this study, a new repeatable method was developed that further examines secondary bone by quantifying the extent to which secondary osteons encroach on previously-existing secondary osteons. Encroached and unencroached secondary osteons were quantified using two different methods: a point count method that measured the percentage of the area the encroached and unencroached secondary osteons inhabited and an osteon count method that measured the number of encroached and unencroached secondary osteons per area. These raw measurements were calculated into 18 parameters and 2-way repeated measures ANOVAs were run to determine the effects of surgery and anatomical region on each of the bone remodeling parameters. The results found significant effects from estrogen deletion which were different depending on if the bone region was predominately in compression or tension. The ovariectomy surgery caused an increase in remodeling, which was mostly confined on the compressive side to areas that have been previously remodeled, but on the tensile side, bone remodeling expanded into areas that used to be primary bone. The new secondary osteons, as a result of the ovariectomy surgery, were larger than in the control animals. There however, was not an increase in porosity from the ovariectomy surgery, which is one of the main characteristics of osteoporosis. The model could be further studied to determine what sheep are doing that prevents them from losing bone and that knowledge could be greatly beneficial for human treatment plans of postmenopausal osteoporosis.

Keywords: Postmenopausal, Osteoporosis, Ovariectomy, Ewe, Bone Remodeling, Encroached Secondary Bone, Unencroached Secondary Bone, Osteonal Encroachment 


\section{ACKNOWLEDGEMENTS}

I would like to thank my advisor Dr. Scott Hazelwood for the opportunity to join his laboratory and work on this project. His courses gave me the foundation that enabled me to conduct this research and find a discipline I enjoy. I would also like to thank Dr. Clifford Les for providing me the opportunity to work on this project and for guiding me along the way with the struggles that research brings. Without your help and patience, this project would not have been possible. I would also like to thank Joe Calcagno and Eric Wong for their previous research with these samples and assistance in becoming familiar with the project. Finally, I would like to thank my family and friends, in particular my parents, for their unending support throughout my time at Cal Poly. Without them, I would not be where I am today. 


\section{TABLE OF CONTENTS}

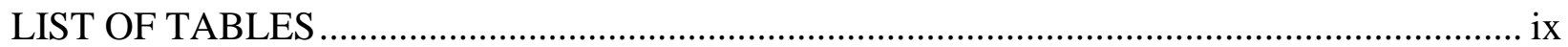

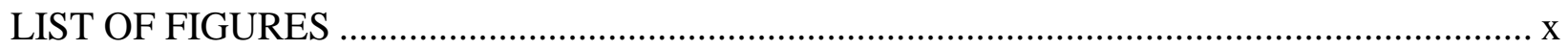

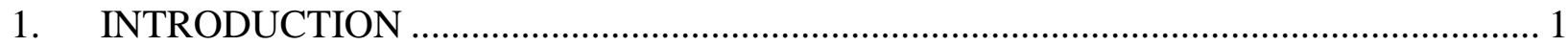

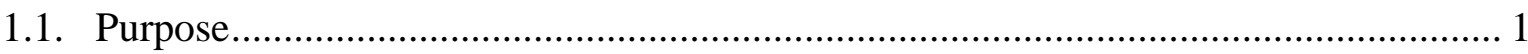

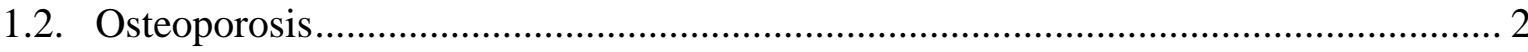

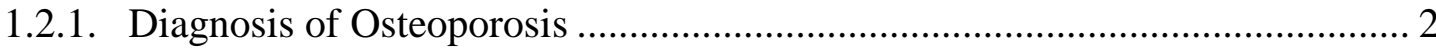

1.2.2. Postmenopausal Osteoporosis and Estrogen............................................... 4

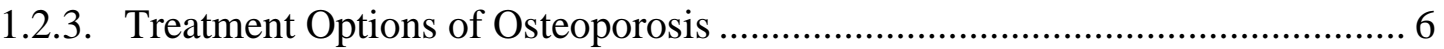

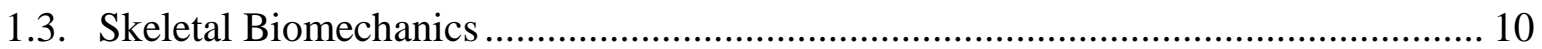

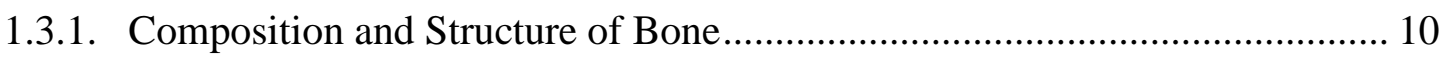

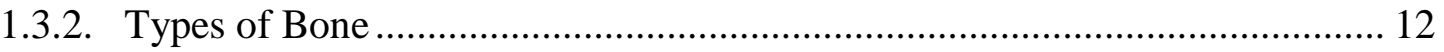

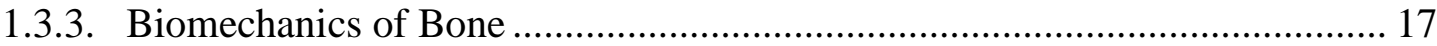

1.3.4. Bone Fracture and Failure Mechanics ..................................................... 18

1.3.5. Quantifying Architectural Features of Bone............................................... 19

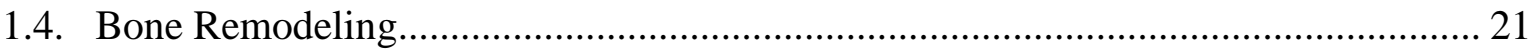

1.4.1. Bone Modeling vs. Bone Remodeling ................................................... 21

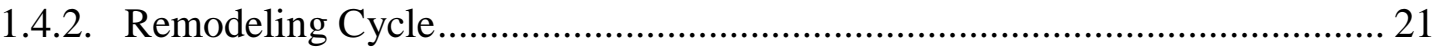

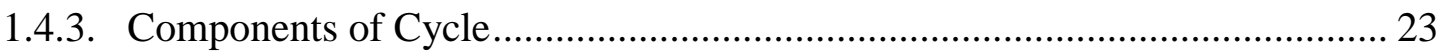

1.4.4. Characteristics of Remodeled Bone ........................................................ 24

1.4.5. Quantifying Remodeled Bone............................................................. 25

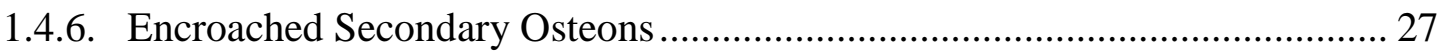

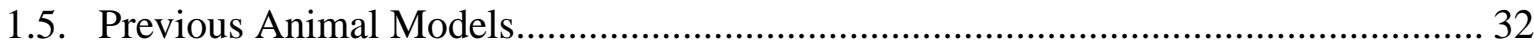

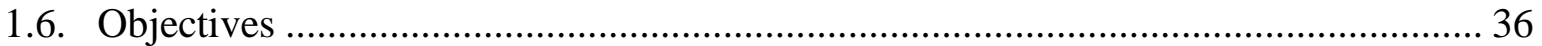

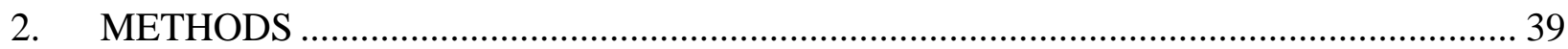

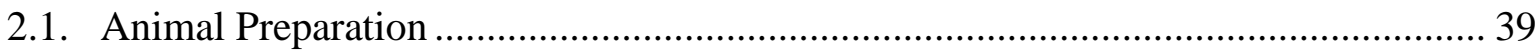

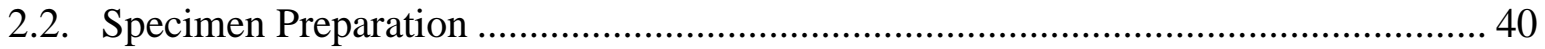

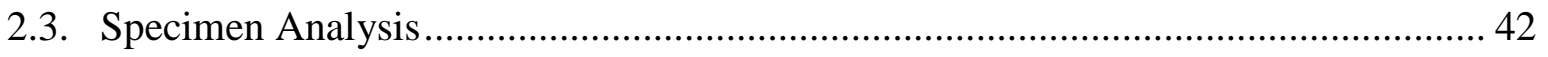

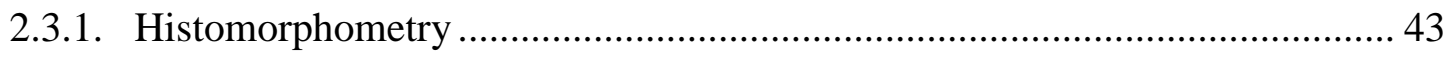

2.3.1.1. Repeatability .......................................................................... 44 
2.3.1.2. Point Count Measurements ............................................................. 45

2.3.1.3. Osteon Count Measurements ........................................................... 48

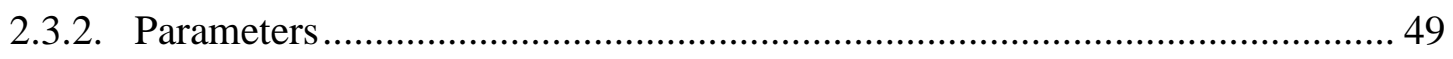

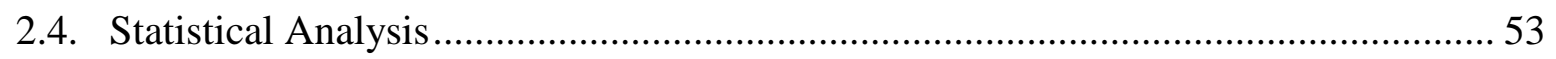

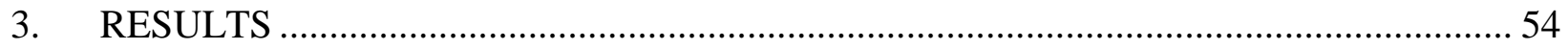

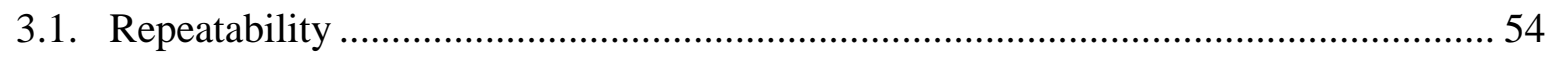

3.2. Two Way Repeated Measures ANOVA …………………………………………..... 54

3.3. Point Count Measurements ................................................................................... 55

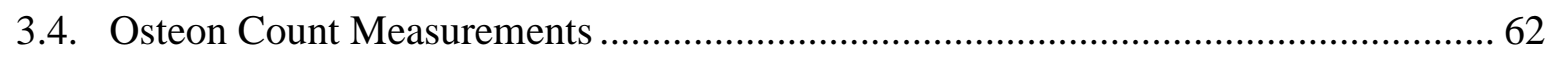

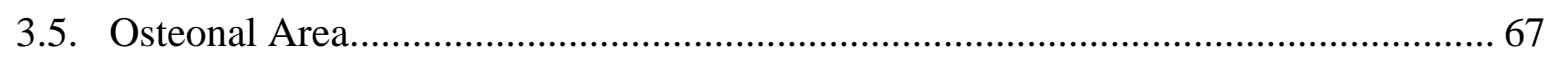

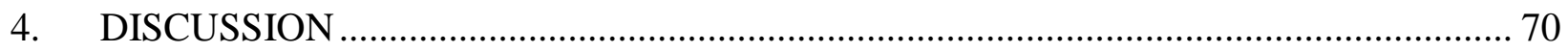

4.1. Postmenopausal Osteoporosis Ovine Model ………................................................. 70

4.2. Hypothesis Evaluation and Results Interpretation ................................................... 70

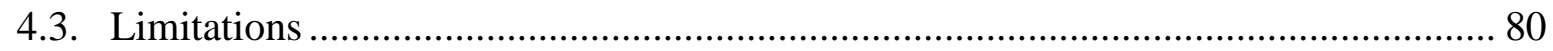

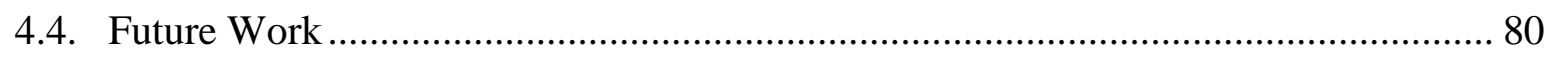

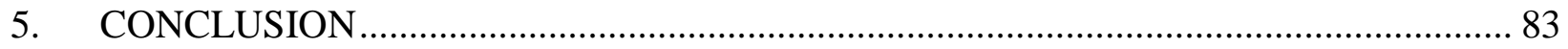

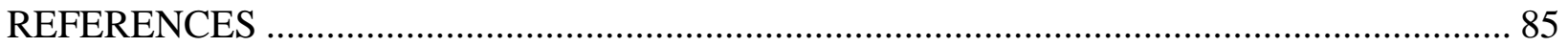

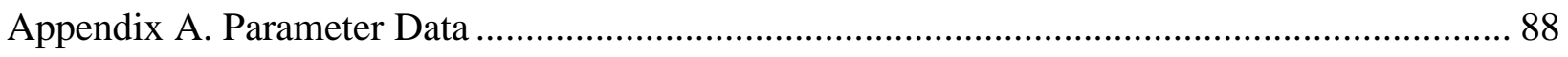




\section{LIST OF TABLES}

Table 1. Table displaying definitions of complete secondary osteons compared to

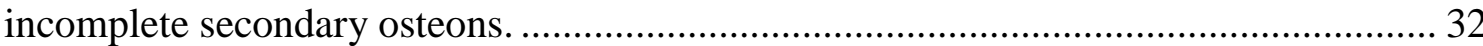

Table 2. P-values found from one way repeated measures ANOVAs determining if there is a significant difference between measurements.

Table 3. P-values found from two way repeated measures ANOVAs for each parameter determining if sector, surgery, or the interaction have a significant effect. 


\section{LIST OF FIGURES}

Figure 1. Scanning electron micrograph of normal bone (left) compared to osteoporotic

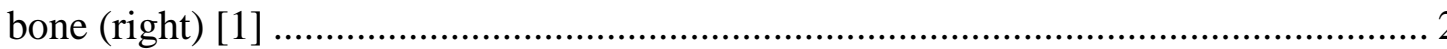

Figure 2. Osteoporotic fractures of the hip, vertebra, and wrist incidence with age in men and women [3]

Figure 3. Regulation of the production of interleukin 1, interleukin 6, and tumor necrosis factor [6] 5

Figure 4. Changes in the mean bone mineral density of the hip and calcaneus due to different estrogen treatment plans with asterisks indicating a significant difference $(p<0.05)$ from the group that never used estrogen treatments [8].

Figure 5. The probability, adjusted for height and weight, of fracturing a hip with different estrogen treatment plans for osteoporosis [8].

Figure 6. Graphs displaying the bone mineral density mean percent change from baseline of (A) the lumbar spine, (B) the total hip, (C) the femoral neck, and (D) the femoral trochanter of 4 different treatment groups: $\mathrm{PBO}$, alendronate, $\mathrm{CE}$, and alendronate and CE [10]

Figure 7. The development of bone cells (a) Osteogenic cells becoming osteoblasts, which become osteocytes (b) Bone marrow stem cells fusing to become osteoclasts [14] .... 12

Figure 8. Structure of cortical long bone [12]

Figure 9. Diagram of unencroached secondary osteons and encroached secondary osteons on a field of primary bone with a resorption cavity present [12]

Figure 10. The ultimate compressive stress, porosity, and apparent density relationship for three types of bone [12].

Figure 11. Merz grid used to measure porosity and other bone remodeling characteristics with histology imaging [17]

Figure 12. Sketch of an osteonal BMU. The small black cells on the left are osteoblasts and the larger white cells on the right are osteoclasts [12]

Figure 13. Photomicrograph displaying the regions of the 6 stages of the osteon's lifetime on a BMU [11]

Figure 14. Bone remodeling diagram: (Top) BMUs in a cross section of cortical bone with (R) resorption spaces, (C) completed osteons, (C,S) completed osteons with a single label, $(\mathrm{F}, \mathrm{S})$ filling BMUs with a single label, and $(\mathrm{F}, \mathrm{D})$ filling BMUs with a double label.(Bottom) An individual BMU in the final stages of filling, with the distance between labels displayed as $\mathrm{D}_{\mathrm{L}}[12]$ 
Figure 15. Microradiograph of equine bone: partial osteons (P), whole osteons (O), and

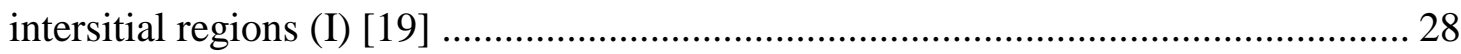

Figure 16. A histological slide with osteon fragments (F) [20] .......................................... 29

Figure 17. A diagram of the cortical bone microstructure with (1) complete secondary osteons, (2) secondary osteons within secondary osteons, (3) secondary osteon fragments, (4) resorption spaces, (5) non-Haversian canals, and (6) primary bone [23].

Figure 18. Histology samples at 25x magnification of (a) 3 year old ewe plexiform bone, and (b) 8 year old ewe Haversian remodeling of the caudal femoral cortext [27]...... 35

Figure 19. Radial-Ulnar approximate anatomy divided into 6 anatomical sectors. The top left sector is the lateral aspect and the top right is the cranial aspect [31] ................. 40

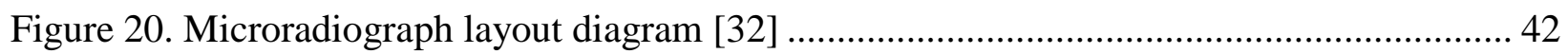

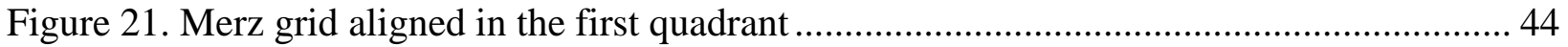

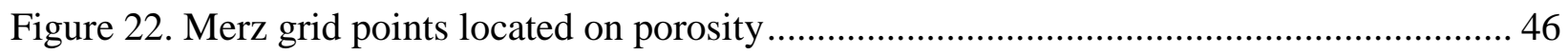

Figure 23. Merz grid points located on primary bone ................................................... 46

Figure 24. Merz grid points located on unencroached secondary bone ................................... 47

Figure 25. Merz grid points located on encroached secondary bone.................................... 47

Figure 26. Another example of Merz grid points located on encroached secondary bone.......... 48

Figure 27. The field of view for the number of encroached secondary osteons and unencroached secondary osteons to be measured

Figure 28. The mean BV/TV with the standard deviation for the sham and OVX sheep for each sector

Figure 29. The mean fraction remodeled as a tissue parameter with the standard deviation for the sham and OVX sheep for each sector....

Figure 30. The mean fraction remodeled as a material parameter with the standard deviation for the sham and OVX sheep for each sector....

Figure 31. The mean fraction encroached as a tissue parameter with the standard deviation for the sham and OVX sheep for each sector....

Figure 32. The mean fraction encroached as a material parameter with the standard deviation for the sham and OVX sheep for each sector....

Figure 33. The mean fraction encroached as a remodeled parameter with the standard deviation for the sham and OVX sheep for each sector....

Figure 34. The mean fraction unencroached as a tissue parameter with the standard deviation for the sham and OVX sheep for each sector 
Figure 35. The mean fraction unencroached as a material parameter with the standard deviation for the sham and OVX sheep for each sector

Figure 36. The mean fraction unencroached as a remodeled parameter with the standard deviation for the sham and OVX sheep for each sector.

Figure 37. The mean number of secondary osteons per tissue area with the standard deviation for the sham and OVX sheep for each sector

Figure 38. The mean number of secondary osteons per material area with the standard deviation for the sham and OVX sheep for each sector....

Figure 39. The mean number of encroached secondary osteons per tissue area with the standard deviation for the sham and OVX sheep for each sector

Figure 40. The mean number of encroached secondary osteons per material area with the standard deviation for the sham and OVX sheep for each sector

Figure 41. The mean number of unencroached secondary osteons per tissue area with the standard deviation for the sham and OVX sheep for each sector

Figure 42. The mean number of unencroached secondary osteons per material area with the standard deviation for the sham and OVX sheep for each sector

Figure 43. The mean secondary osteon average area with the standard deviation for the sham and OVX sheep for each sector.

Figure 44. The mean encroached secondary osteon average area with the standard deviation for the sham and OVX sheep for each sector

Figure 45. The mean unencroached secondary osteon average area with the standard deviation for the sham and OVX sheep for each sector....

Figure 46. A cross section of cortical bone from the mid shaft of a normal human femur displaying anatomical differences in osteonal density between (a) the inner side, in compression, and (b) the outer side, in tension [35]

Figure 47. Compact bone yield strength and stiffness for the OVX compared to control groups [36]

Figure 48. An approximate illustration of how the cumulative area, number, and average size of unencroached and encroached secondary osteons differ between groups based on the effect of sham vs. OVX and compression vs. tension. 


\section{INTRODUCTION}

\subsection{Purpose}

Osteoporosis is a major health concern in the United States, on average about 40 million Americans live with osteoporosis [1]. Osteoporosis is a decrease in bone mass, which usually happens with age [1]. This is because bone resorption increases and bone formation decreases after the age of 30 [2]. Osteoporosis typically affects older women more so than any other group, one major cause of osteoporosis is linked to decreased levels of estrogen post-menopause [3]. With decreased bone mass, individuals are more susceptible to fractures within their bone as well [2]. These fractures can greatly decrease the quality of life for individuals with osteoporosis. The United States spends \$18 billion each year to treat bone fractures caused by osteoporosis [1]. Individuals that fracture a bone when they have osteoporosis also have $35 \%$ chance of death post fracture depending on the location it occurs [3].

Research has been and remains focused on the prevention, treatment, and reversal of osteoporosis. The purpose of this thesis is to study the Columbia-Rambouillet sheep that have been ovariectomized as a model for postmenopausal osteoporosis. The study will quantify certain characteristics of bone remodeling in the cortical bone of the sheep of both ovariectomized and control sheep. It will determine a new repeatable method that further examines secondary bone by quantifying the extent to which secondary osteons encroach on previously-existing secondary osteons. The introduction will provide background information relevant to this research: including information on osteoporosis, the biology and mechanical properties of bone, the remodeling process that bone undergoes, previous animal models for postmenopausal osteoporosis, and previous studies conducted on these specimens. 


\subsection{Osteoporosis}

\subsubsection{Diagnosis of Osteoporosis}

Osteoporosis affects $55 \%$ of individuals over the age of 50 in the United States, with the majority of people affected being postmenopausal women [1]. Osteoporosis is diagnosed by a decreased bone mass and a damaged micro-architecture [1]. Another way to describe this disease, more commonly found in patients with old age, is the thinning of bone tissue leading to bone density loss [3]. Osteopenia is not as severe of a bone loss compared to osteoporosis, but it is typically the precursor for it. Figure 1 shows a scanning electron micrograph of the difference in microarchitecture between normal bone of the L3 vertebra of a 31 year old women, on the left, and osteoporotic bone of a 70 year old women, on the right [3]. The osteoporotic bone shows an increased porosity and the trabecular plate-like struts diminished to thin rods. Osteoporosis is either due to resorption of too much old bone or failure to make enough new bone to replace damaged bone. In other words, osteoporosis could be from an increased activity of osteoclasts or a decreased formation from osteoblasts [3].

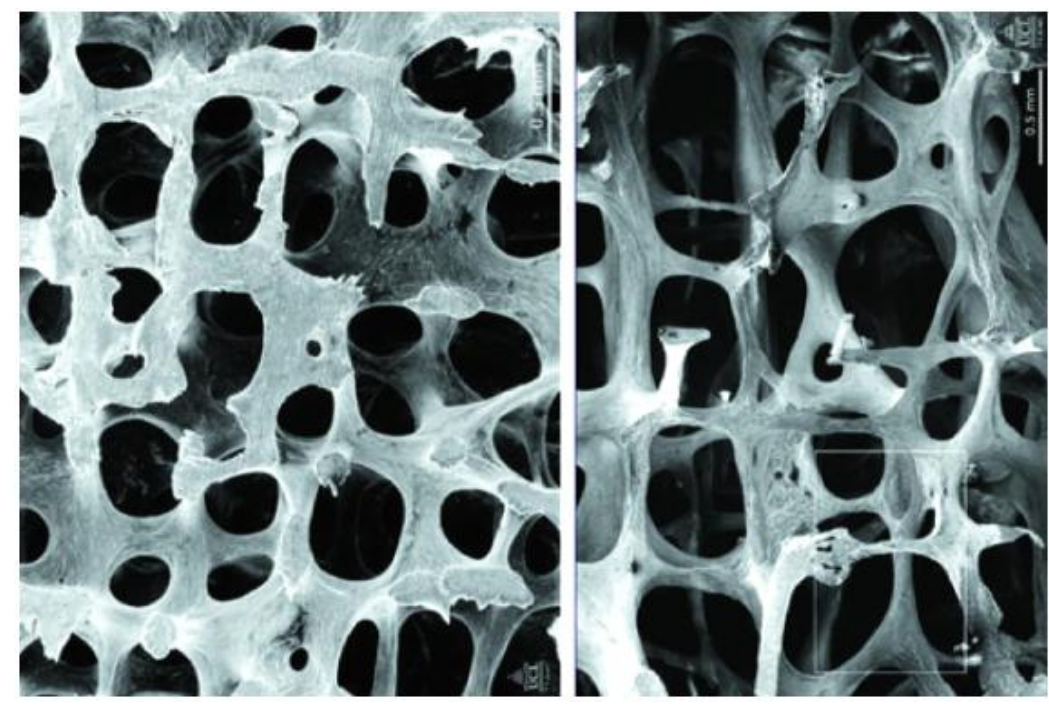

Figure 1. Scanning electron micrograph of normal bone (left) compared to osteoporotic bone (right) [1]. 
It has been shown that individuals with osteoporosis are at an increased risk of bone fracture [2]. It was estimated that in 2000, 5.8 million people had osteoporotic fractures which caused disability and an increased risk of mortality [3]. Fracture risk increases dramatically with age, especially after the age of 75, and these fractures typically occur in the spine, pelvis, hip, humerus, and wrist [3]. Figure 2 graphically displays the increase in fractures in the hip, vertebra, and wrist for both men and women. Reasons women experience a higher occurrence of fractures include: a 20-30\% lower peak bone mass compared to men and the decrease in estrogen from menopause [3]. Typically, peak bone mass occurs during the late teens to early twenties of an individual's lifetime, it remains constant until the forties, and it beings to drop off after that [1].

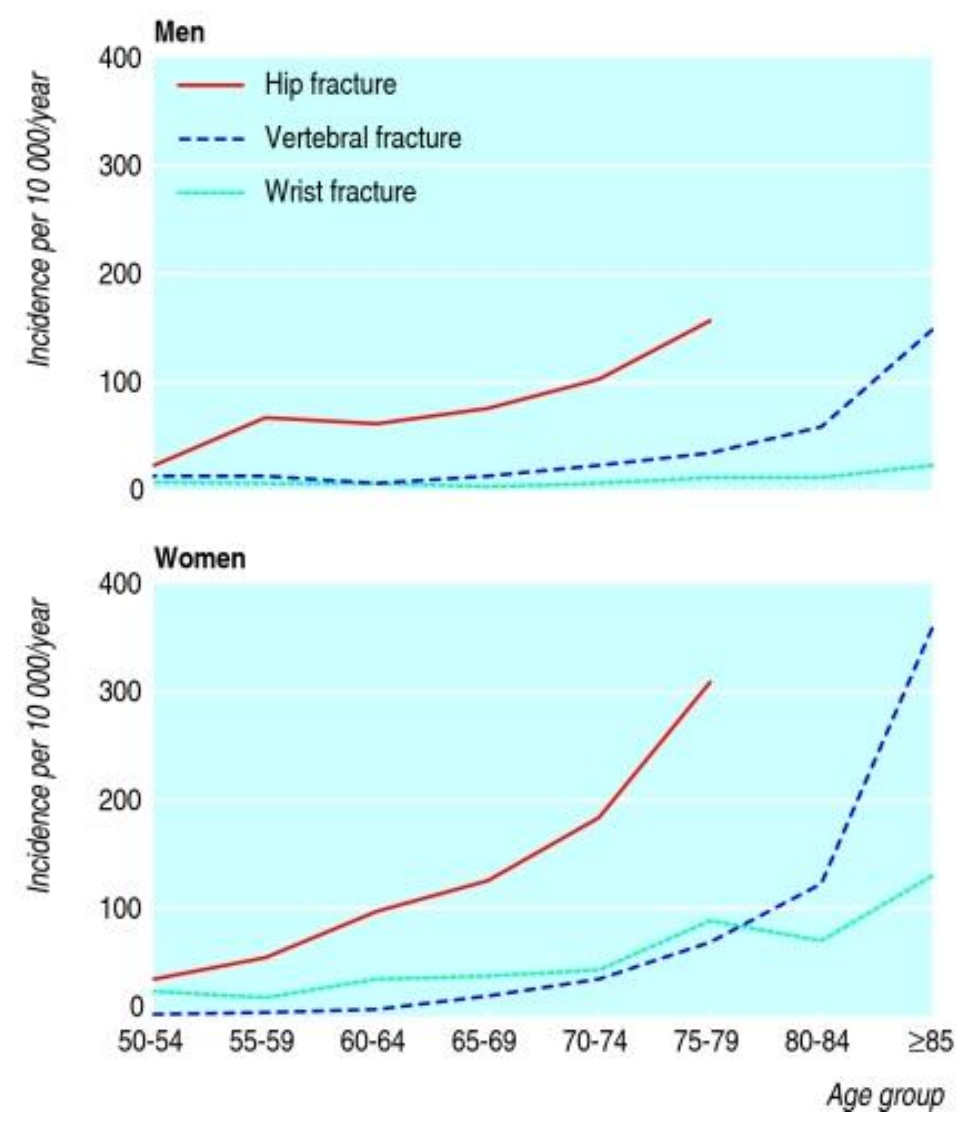

Figure 2. Osteoporotic fractures of the hip, vertebra, and wrist incidence with age in men and women [3]. 
Risk factors of osteoporosis can come from an individual's characteristics being female, elderly, thin boned, Caucasian, or Asian [1]. There have also been links between osteoporosis and reduction of physical activity with age [3]. Other effectors of osteoporosis include genetics of bone mass, family history of fractures, and peak bone mass obtained earlier in life [1]. Lifestyle choices can also increase the risk of osteoporosis which includes: excessive alcohol consumption, anorexia, cigarette smoking, sedentary daily life, corticosteroid use, and a diet deficient in calcium and/or vitamin D [1]. Additionally, if the liver produces a decreased amount of insulin-like growth factor, it may lead to osteoporosis [3]. Low levels of sex hormones, estrogen in females and testosterone in men, are also a huge precursor [1]. This explains why osteoporosis is most commonly seen in postmenopausal women; there is a dramatic change in hormones resulting in a loss in estrogen content [3].

\subsubsection{Postmenopausal Osteoporosis and Estrogen}

Menopause is described as a time in a women's life when she no longer goes through menstruation cycles because she stops extruding eggs. This occurs because the ovaries no longer respond to the follicle stimulating hormone (FSH) and the luteinizing hormone $(\mathrm{LH})$ secreted by the pituitary gland, which prior to menopause caused the production of estrogen, progesterone, and testosterone [4]. The loss in bone in osteoporosis can be related to these decreased levels in estrogen in postmenopausal women [3]. 30\% of postmenopausal women will have osteoporosis initially, and 70\% of women will have osteoporosis by the age of 80 [5].

Estrogen has been associated with stimulation of osteoclasts, which is due to estrogen's regulation of cytokines. Some of the cytokines that estrogen regulates include interleukin 1 (IL1), interleukin 6 (IL-6), and tumor necrosis factor (TNR) [6]. Among the effects of these 
cytokines is to regulate osteoclastogenesis. Interleukin 6 for example can help control formation of osteoclasts, which is increased with a deficiency in estrogen or decreased when estrogen levels are large enough [6]. Figure 3 shows how estrogen regulates interleukin 1, interleukin 6 , and tumor necrosis factor. Postmenopausal women show increased levels of interleukin 1, interleukin 6, and tumor necrosis factor. Together, the increase in these factors can be related to prolonged life of the osteoclasts [6].

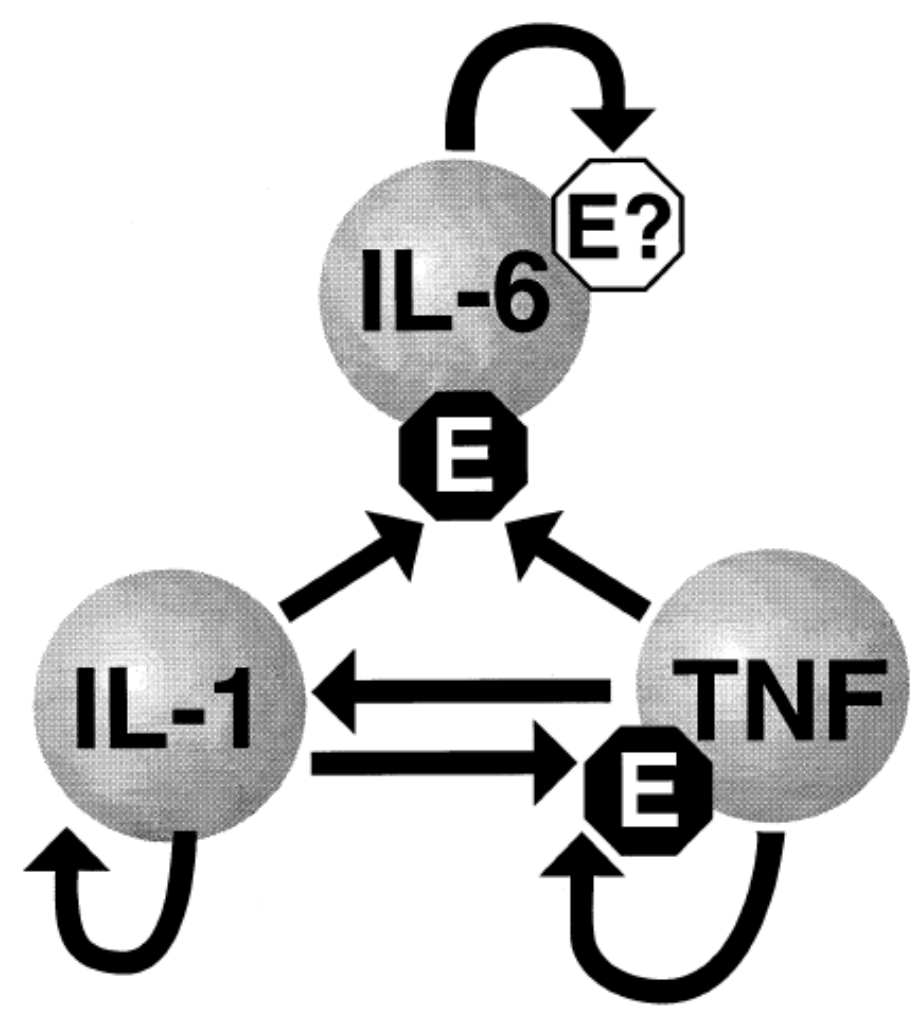

Figure 3. Regulation of the production of interleukin 1, interleukin 6, and tumor necrosis factor [6].

Transforming growth factor- $\beta$ (TGF- $\beta$ ) is also stimulated by estrogen, but in cells of osteoblasts, which stimulates osteoclast apoptosis [6]. In Figure 3, the arrows indicate synthesis of a different cytokine or a stimulatory effect of a cytokine on its own synthesis. Estrogen, shown as a black octagon labeled "E", suppresses the stimulated synthesis of interleukin 6 and tumor 
necrosis factor. Estrogen, shown as a white octagon labeled "E", potentially suppress interleukin 6 which is induced by interleukin 6 by estrogen. A significant increase in any of these cytokines depends on the presence of the other cytokines, due to the interdependent regulatory circuit. This apoptosis is important so that resorption can stop so formation can begin. When a woman is in postmenopause, she most likely has an increase in interleukin 1, interleukin 6 , and tumor necrosis factor, but a decrease in transforming growth factor- $\beta$ because of the lower levels of estrogen [6]. This leads to an expanded life time of the osteoclasts, leading to an increase in resorption.

\subsubsection{Treatment Options of Osteoporosis}

In order to determine if an individual has osteoporosis or osteopenia, a Dual energy x-ray absorptiometry (DEXA) scan is taken on individuals. This will determine the bone mineral density (BMD) so a treatment plan can be can be determined [7]. There are lifestyle measures that can be taken to help improve bone health which include: obtaining healthy levels of vitamin $\mathrm{D}$, having a sufficient intake of calcium, exercising regularly, refraining from smoking, and not drinking alcohol in excess [3]. But when that is not enough, there are two of main treatment options for osteoporosis which include increasing estrogen levels or delivering of bisphosphonates. Although both show decreased loss of BMD [8], estrogen has bad side effects

for the patient [9] and bisphosphonates alter osteoclasts function negatively [10]. There has been some research done to show a positive effect combining both treatment options, but further research is needed [10].

Since decreased levels of estrogen lead to continual production of cytokines stimulating osteoclastogenesis and decreased levels of other cytokines (TGF- $\beta$ ) that increase osteoclasts 
apoptosis, giving patients estrogen seems like a good option. One study looked at the effects in bone mineral density and probability of fractures in patients with osteoporosis from four different treatment plans: continuous users of estrogen, partial users of estrogen, past users of estrogen, and non users of estrogen [8]. Figure 4 and Figure 5 show results from a study that looked at the effectiveness of treating osteoporosis by taking estrogen. The results showed that patients that had a treatment plan of partial or continuous use of estrogen decreased the bone mineral density loss and deceased the probability of fractures [8]. However, this study noted that patients who took estrogen sometimes experienced headaches, dyspepsia, and mastalgia [8]. Another issue, as seen in Figure 4, when a patient stops taking estrogen to help decrease the loss in bone mineral density, their bone mineral density drops to worse than if they never took estrogen. Figure 5 shows the treatment will just delay the increase of fracture probability rather than preventing it. 


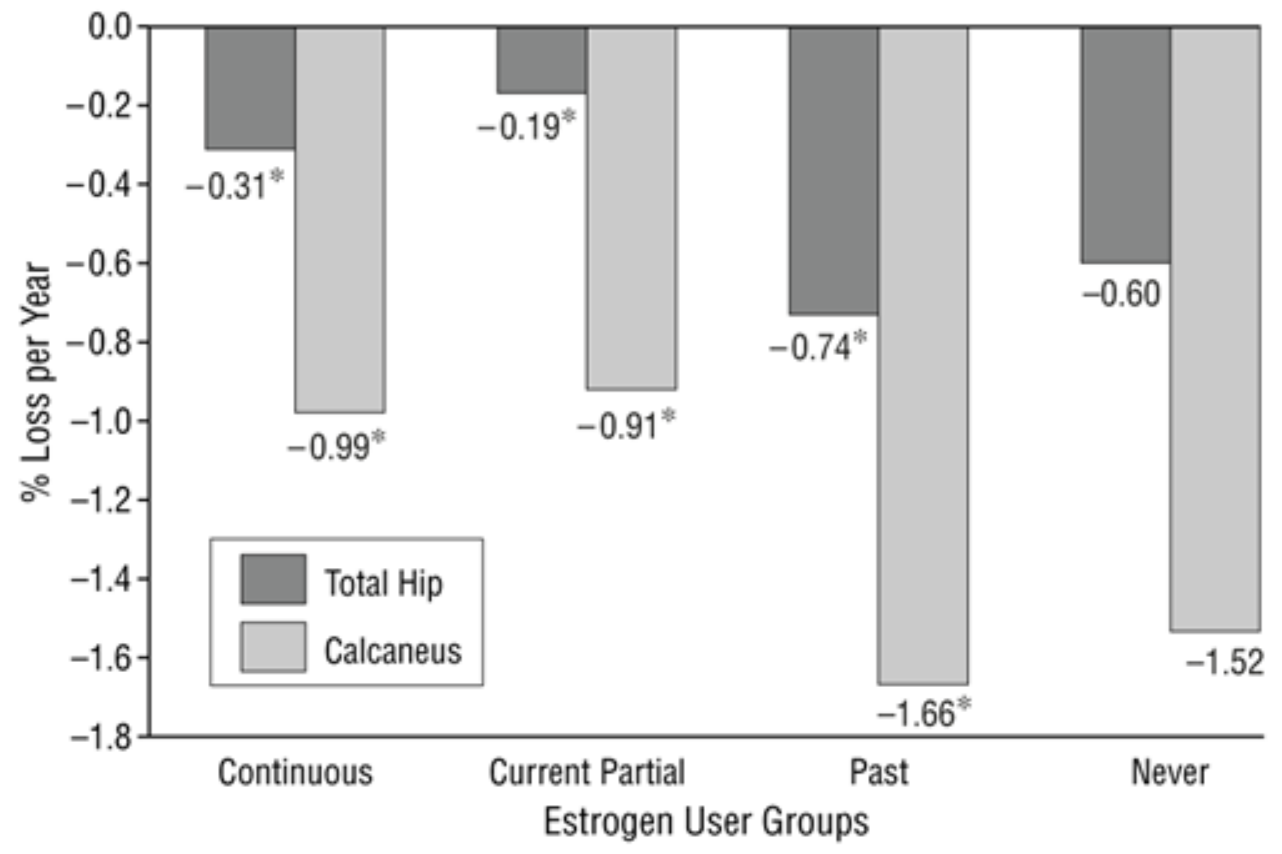

Figure 4. Changes in the mean bone mineral density of the hip and calcaneus due to different estrogen treatment plans with asterisks indicating a significant difference $(\mathrm{p}<0.05)$ from the group that never used estrogen treatments [8].

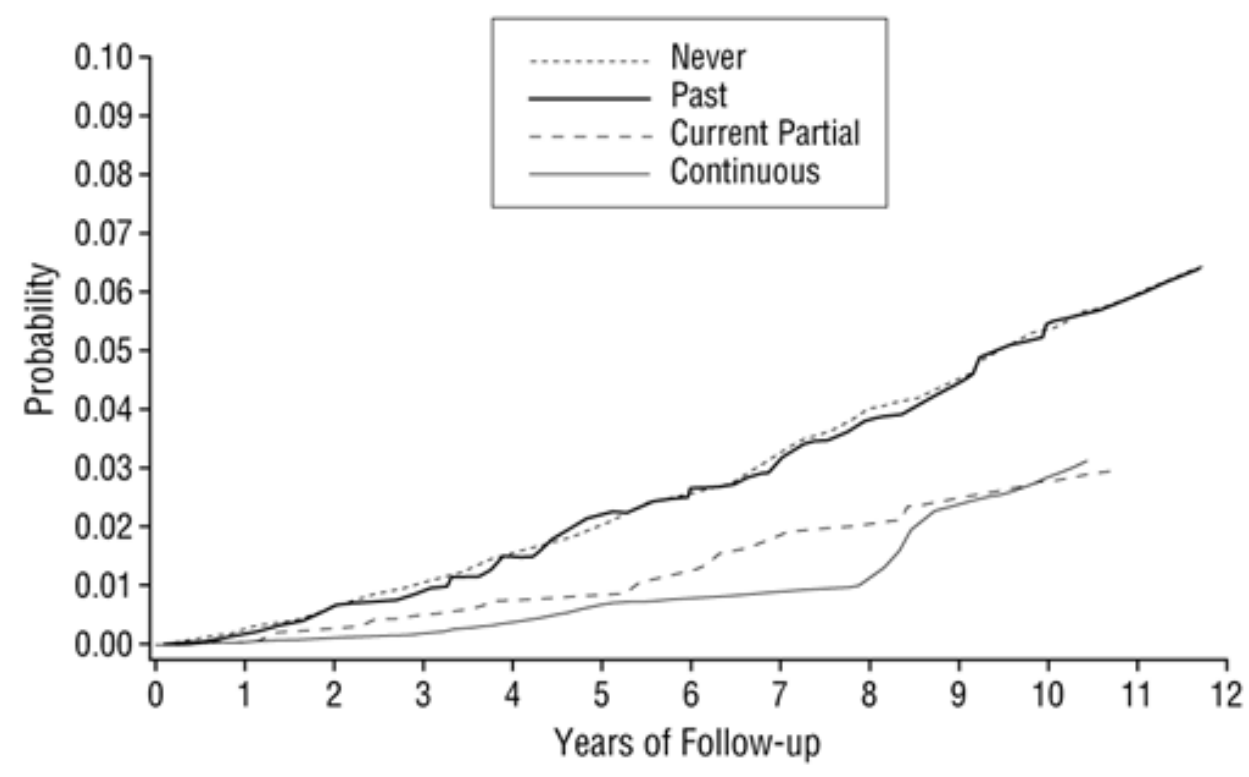

Figure 5. The probability, adjusted for height and weight, of fracturing a hip with different estrogen treatment plans for osteoporosis [8]. 
Bisphosphonates work by suppressing bone resorption, or inhibiting osteoclasts [9]. They have shown to reduce vertebral and non-vertebral fractures so they are normally one of the first options in treating osteoporosis, especially in men where estrogen therapy is not an option [3]. One side effect of bisphosphonates includes upper gastrointestinal issues [3]. Another issue with bisphosphonate treatments is that even when treatment stops being delivered, the treatment still is occurring due to their potential sequestering by hydroxyapatite crystals [9]. They have also been found to inhibit the melavonic pathway, an important aspect for osteoclasts to function [9]. The problem with decreasing osteoclasts function is that in remodeling, osteoclasts and osteoblasts are coupled together. This means that there is less bone turnover compared to before, so even though there is less bone loss, the bone which is present most likely is worse quality bone with microfractures and other defects present [9]. This can lead to more fractures later even though the bone mineral density is sufficient.

Other studies have looked at the effects when both bisphosphonates and estrogen are used as a combined treatment plan [10]. In this study, alendronate, a bisphosphonate, was used with conjugated equine estrogen (CE), along with three control groups: a placebo, just alendronate, and just conjugated equine estrogen [10]. The effects on bone mineral density were how these treatments were analyzed. Figure 6 shows the bone mineral density changes for each group, which were that the combined treatment showed the lowest decrease in bone mineral density compared to the other groups. This difference was only a little improvement from the individual treatments. 

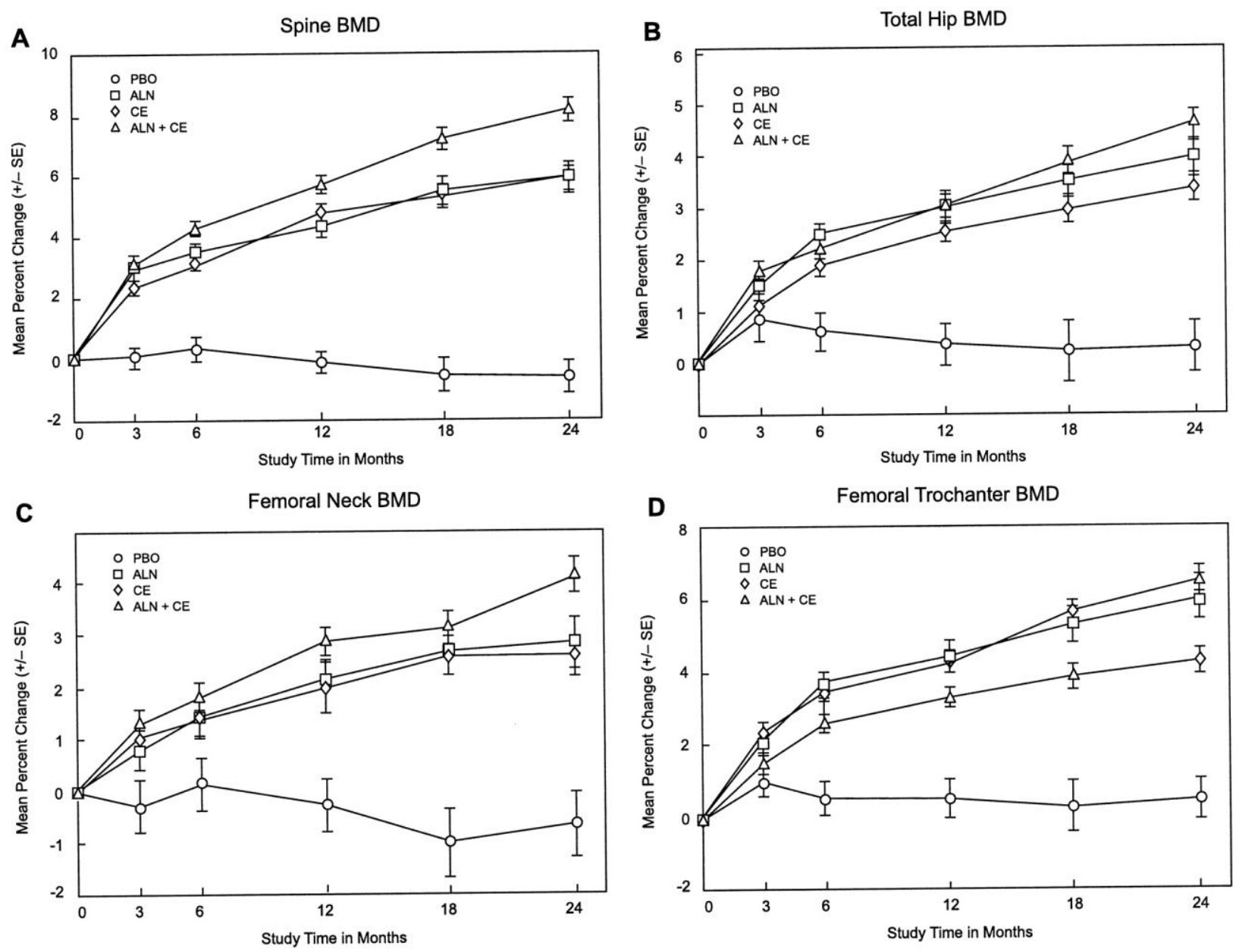

Figure 6. Graphs displaying the bone mineral density mean percent change from baseline of (A)

the lumbar spine, (B) the total hip, (C) the femoral neck, and (D) the femoral trochanter of 4 different treatment groups: PBO, alendronate, CE, and alendronate and CE [10].

\subsection{Skeletal Biomechanics}

\subsubsection{Composition and Structure of Bone}

The main functions of bone are to provide structural support, serve as a reservoir of calcium, protect vital organs, house bone marrow which is the source of blood cells and stem cells, and aid in motion $[11,12,13]$. Bone is a dynamic tissue which changes its composition and properties based on the environment it experiences. With increased forces exerted on the bone, 
the bone then modifies its structure to withstand the load and prevent fractures from occurring [13].

The extracellular matrix is one of that factors which determines the properties of bone. The extracellular matrix is composed of $70 \%$ hydroxyapatite, $18 \%$ collagen, $2 \%$ proteoglycans, and $10 \%$ water $[11,12,13]$. Bone also consists of noncollagenous proteins including osteocalcin, osteonectin, and osteopontin, which all make up less than $1 \%$ of the extracellular matrix [12]. The collagen, which is mostly type I, provides the tensile strength and flexibility [12]. The collagen also serves as a site for mineralization; the mineral in bone mainly consists of hydroxyapatite crystals which provide the compressive strength [12]. Proteoglycans serve as the ground substance of the bone. Most of the water content in the extracellular matrix is bounded to collagen, but the content of water in bone reduces as it mineralizes [11].

The cells within bone include osteoprogenitor cells, osteoclasts, osteoblasts, bone lining cells, and osteocytes [12]. The osteoprogenitor cells are mesenchymal cells which include both preosteoclasts and preosteoblasts [12]. The osteoclasts are multinucleated giant cells that have the purpose of resorbing bone, while osteoblasts have a single nucleus, are cuboidal in shape, and have the purpose of forming bone [12]. Bone lining cells are quiescent osteoblasts that line the surface between osteocytes. Osteocytes are cells which were also previously osteoblasts that have been buried in the bone matrix [11]. These types of bone cells and their development can be seen below (Figure 7). 


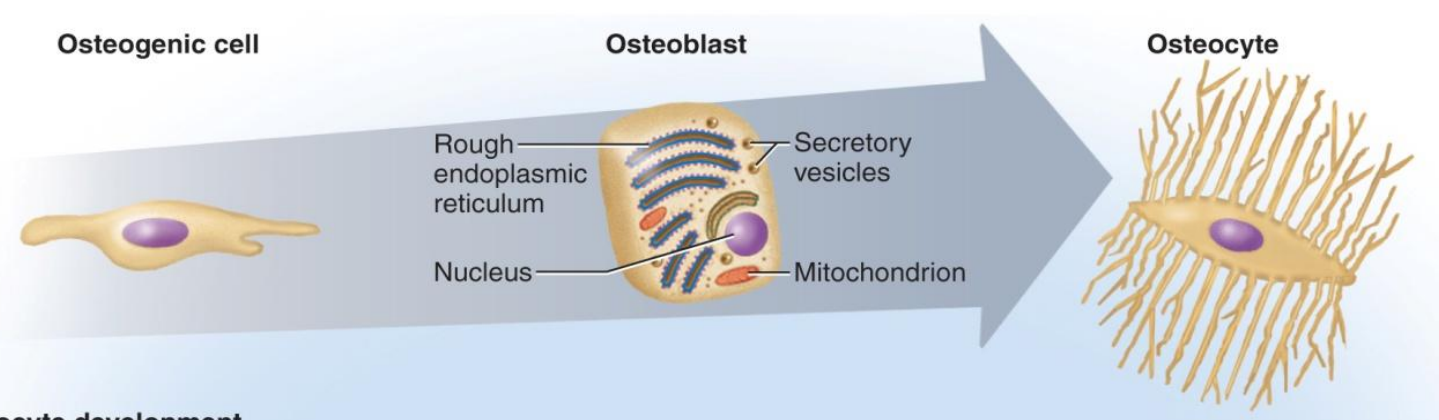

(a) Osteocyte development

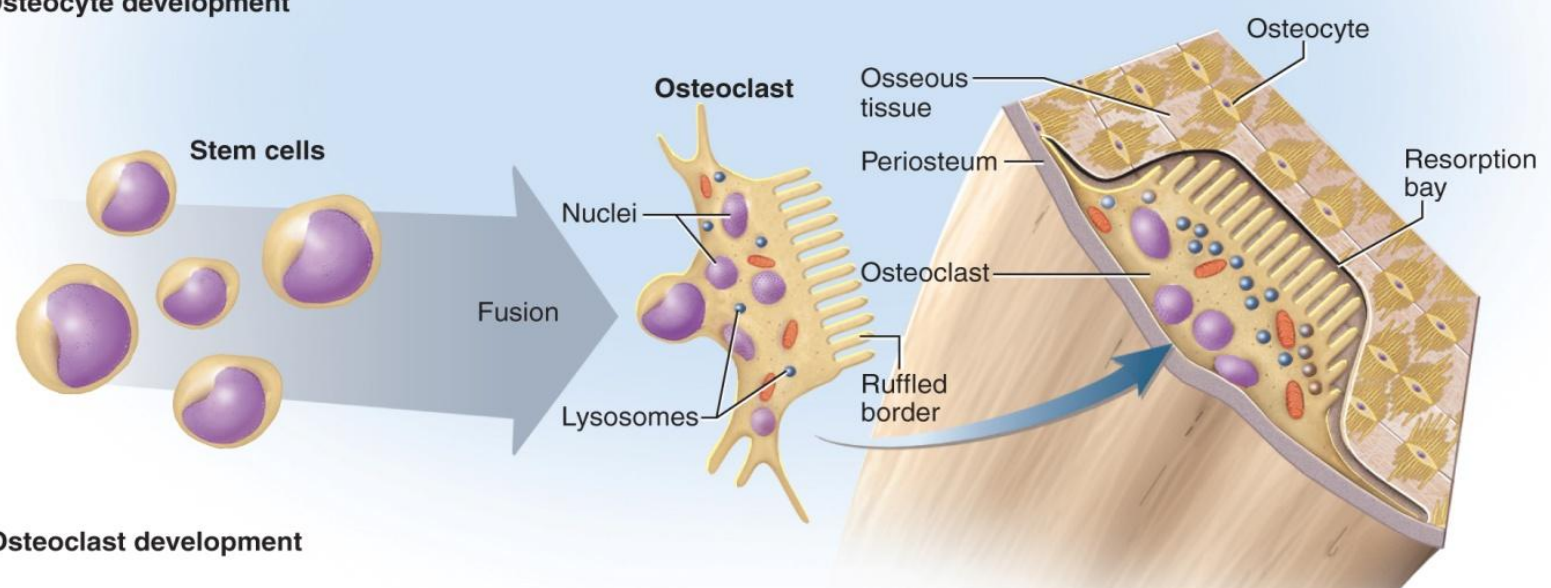

Figure 7. The development of bone cells (a) Osteogenic cells becoming osteoblasts, which become osteocytes (b) Bone marrow stem cells fusing to become osteoclasts [14].

\subsubsection{Types of Bone}

There are two main types of bone: trabecular and compact bone. Trabecular bone, also called cancellous or spongy bone, is porous bone usually with a porosity around $75 \%$ to $95 \%$ [12]. Trabecular bone is located in flat bones, the end of long bones, and cuboidal bones such as vertebral bodies [12]. This type of bone looks like randomized interconnected plates and struts, referred to as trabeculae, which are 200 micrometers thick [12]. Bone marrow also fills the pores of trabecular bone [12].

Cortical bone, also called compact, is dense bone that is usually around $5 \%$ to $10 \%$ porous, but is defined as porosity up to $30 \%$ [15]. Porosities larger than $30 \%$ can be defined as 
trabecular bone [15]. Cortical bone is located in the shafts of long bone and as a shell around some trabecular bone. The pores found in cortical bone are from one of three structures: Haversian canals, Volkmann's canals, or resorption cavities [12]. In humans, Haversian canals have a diameter of about 50 micrometers, contain nerves and blood vessels, and are oriented in the bone's long axis [12]. Volkmann's canals connect the Haversian canals together. Volkmann's canals are transverse to the long axis of the bone and shorter, but they also contain blood vessels and nerves. Resorption cavities are 200 micrometers in diameter and are a result of spaces left from osteoclasts in the beginning of a remodeling stage [12]. These characteristics of porous features in cortical bone can be seen in Figure 8. 


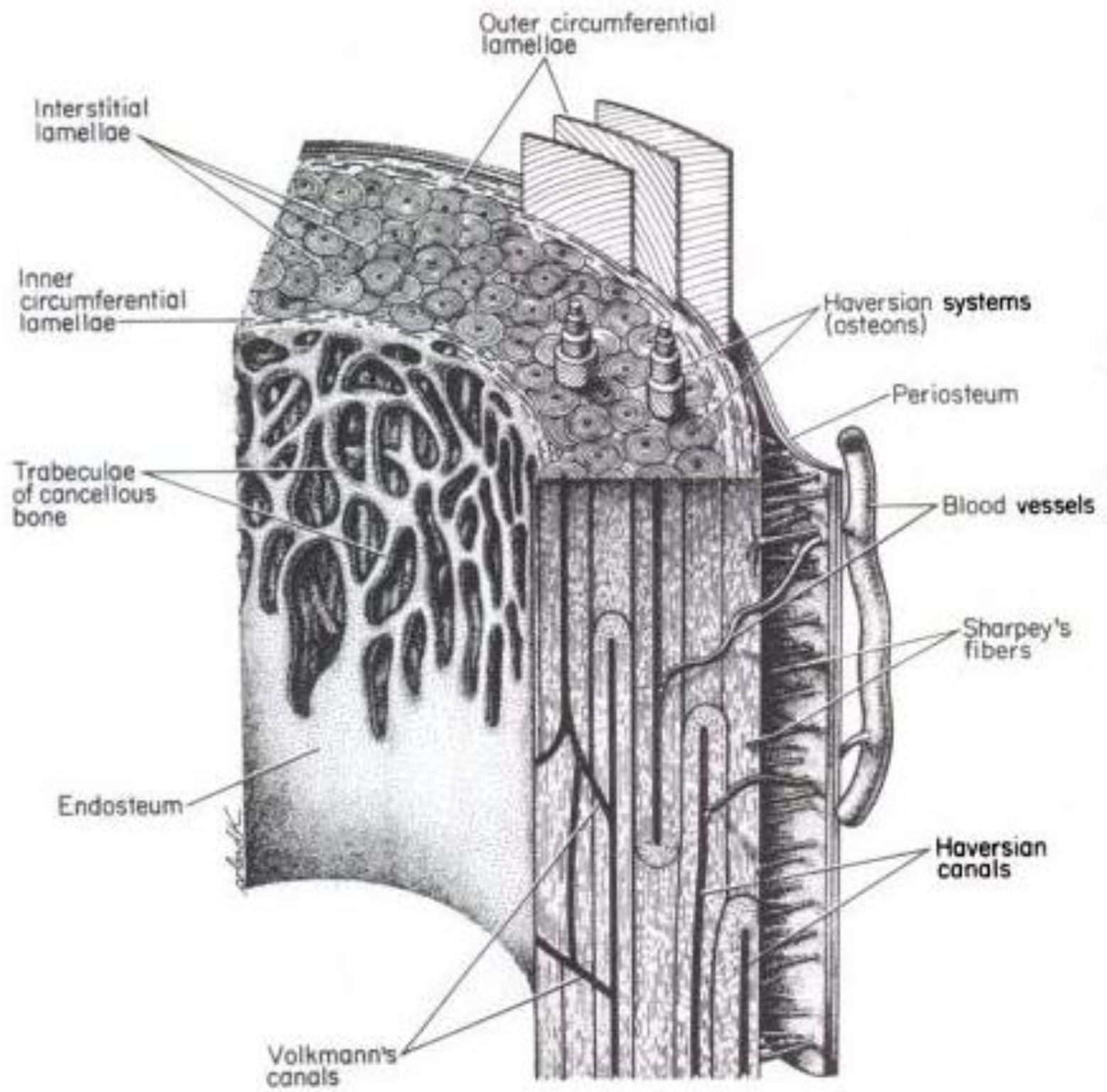

Figure 8. Structure of cortical long bone [12].

Bone tissue can also be classified on a finer resolution scale which includes lamellar and woven bone. Lamellar bone is very organized bone and slowly formed [12]. It is composed of lamellae, or parallel layers, which are composed of collagen fibers and mineral crystals in an anisotropic bone matrix [12]. There are different orientations of lamellar bone which include one architecture type, referred to as the classical view, where in each lamella the collagen fibers are 
parallel, but change by ninety degrees at the lamellar interfaces. Another type of architecture, which can be called helicoidal plies, is when there is not individual lamella since the collagen fibers continuously change their direction [12]. Woven bone is poorly organized, quickly formed, and typically weaker than lamellar bone [12]. Woven bone usually becomes more mineralized than lamellar bone [12].

Another classification can be made to distinguish bone is whether the bone is primary bone or secondary bone. Primary bone is the original bone laid down during growth which can either be circumferential lamellar bone or plexiform bone. Circumferential lamellar bone is bone where the lamellae are parallel to the surface of the bone [12]. When a blood vessel is incorporated into the lamellar bone, a primary osteon is formed which includes a primary Haversian canal in the center [12]. Plexiform bone occurs during rapid growth to fill in gaps and make surface trabecular networks. Plexiform bone is when the lamellar bone mixes with woven bone, which displays a brick like appearance [12].

Secondary bone is the result of resorption and replacement of bone. Secondary osteons, or Haversian systems, are created when new lamellar bone replaces older lamellar bone in the remodeling process [12]. Haversian systems can be seen in Figure 8 in a sketch of cortical long bone. These structures have a diameter around 200 micrometers and about 16 cylindrical lamellae which surround the Haversian canal [12]. Cement lines are present in secondary bone between the osteon and the other surrounding bone. In adults, most cortical bone is composed of all secondary bone, which includes both whole secondary osteons and interstitial bone, also referred to as encroached secondary osteons or partial secondary osteons, which have been incompletely resorbed (Figure 9) [12]. This thesis examines cortical bone by classifying secondary bone as either unencroached secondary osteons (whole osteons) or encroached 
secondary osteons. Trabecular bone in adults is consistent with cortical bone in that it is made up of secondary bone, however osteons rarely occur. Trabecular bone mostly occurs at the surface and is mostly remodeled at a rate faster than cortical bone [12].

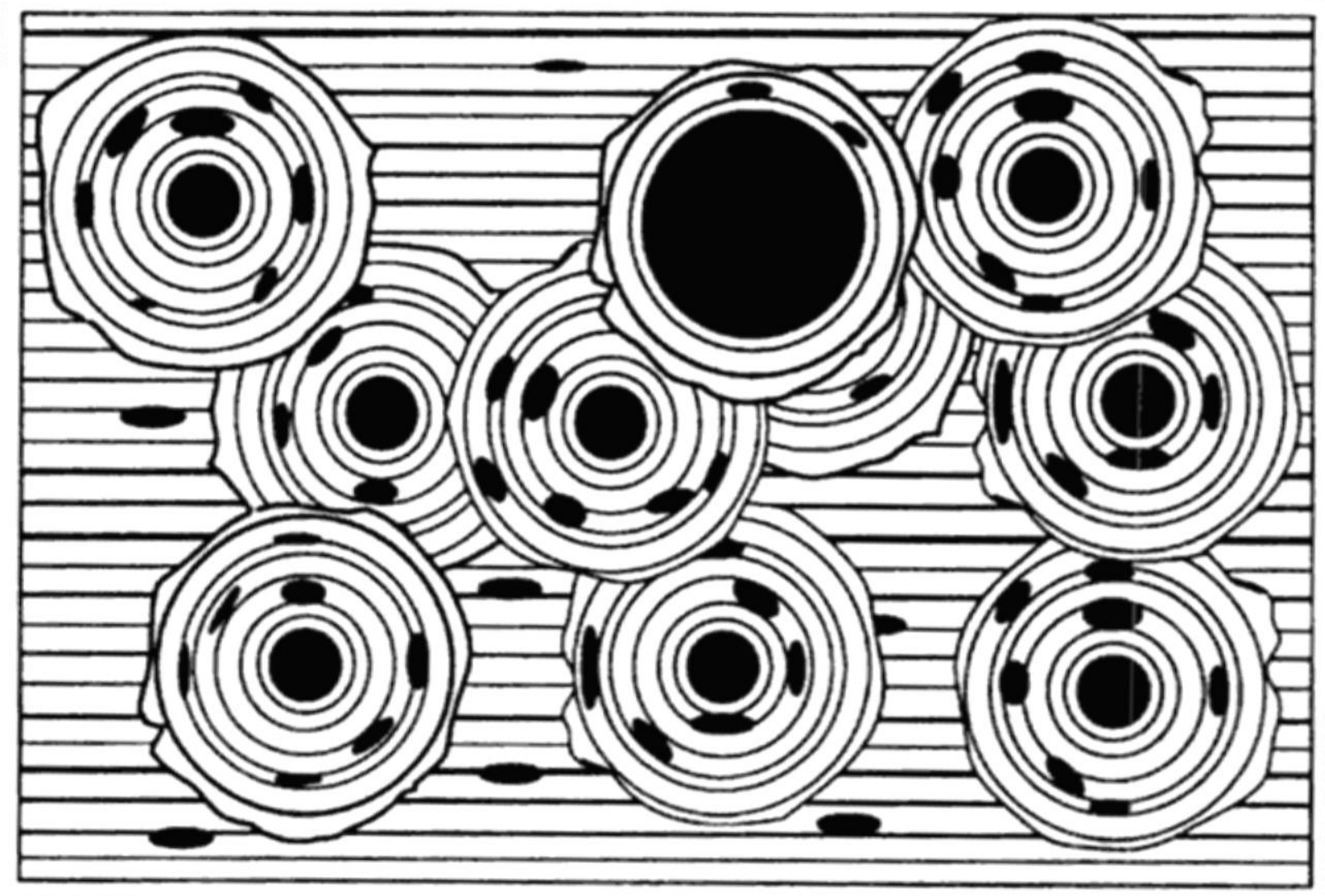

Figure 9. Diagram of unencroached secondary osteons and encroached secondary osteons on a field of primary bone with a resorption cavity present [12].

Secondary osteons are a resultant of bone remodeling in cortical bone [12]. Multiple secondary osteons can also be seen in Figure 8 and Figure 9. They are cylindrical shaped structures which are formed by concentric lamellae and aligned with the bone long axis [12]. The dimensions are 200 micrometers in diameter and 5 to 10 millimeters long [12]. They contain blood vessels and nerves in the center, located in the Haversian canal [11]. 


\subsubsection{Biomechanics of Bone}

Since bone is a highly complex material from all of the hierarchical components discussed above, bone is best modeled as a fiber reinforced composite [12]. Macroscopically the fibers are the osteons and the matrix is the primary bone [12]. Microscopically the fiber is the collagen and the matrix is the hydroxyapatite. This can explain why bone is strong in compression because of the composite material, and how it can support some tension in the longitudinal direction from the fibers. However, the fibers in this model, meaning the osteons and collagen, are not very strong since bone cannot undergo strong tensile forces.

Most bones in the skeletal system often experience compressive and longitudinal forces rather than tensile and transverse forces. Due to the orientation of collagen and osteons in cortical bone, bone exhibits strong anisotropic properties [13]. Cortical bone also exhibits viscoelastic properties. These properties include strain rate dependent, hysteresis, stress relaxation, and creep [11]. These properties and the mechanical strength can be greatly reduced by voids in the bone [12]. The majority of the pores are from Haversian canals, Volkmann's canals, and pores which have a detrimental effect on strength [12]. Figure 10 displays this relationship between strength and porosity from three different types of bone, showing a drastic decrease in strength with small decreases in porosity when the porosity is greater than 0.1 . The relationship then shows a gradual decrease in ultimate stress from decreasing porosity. 


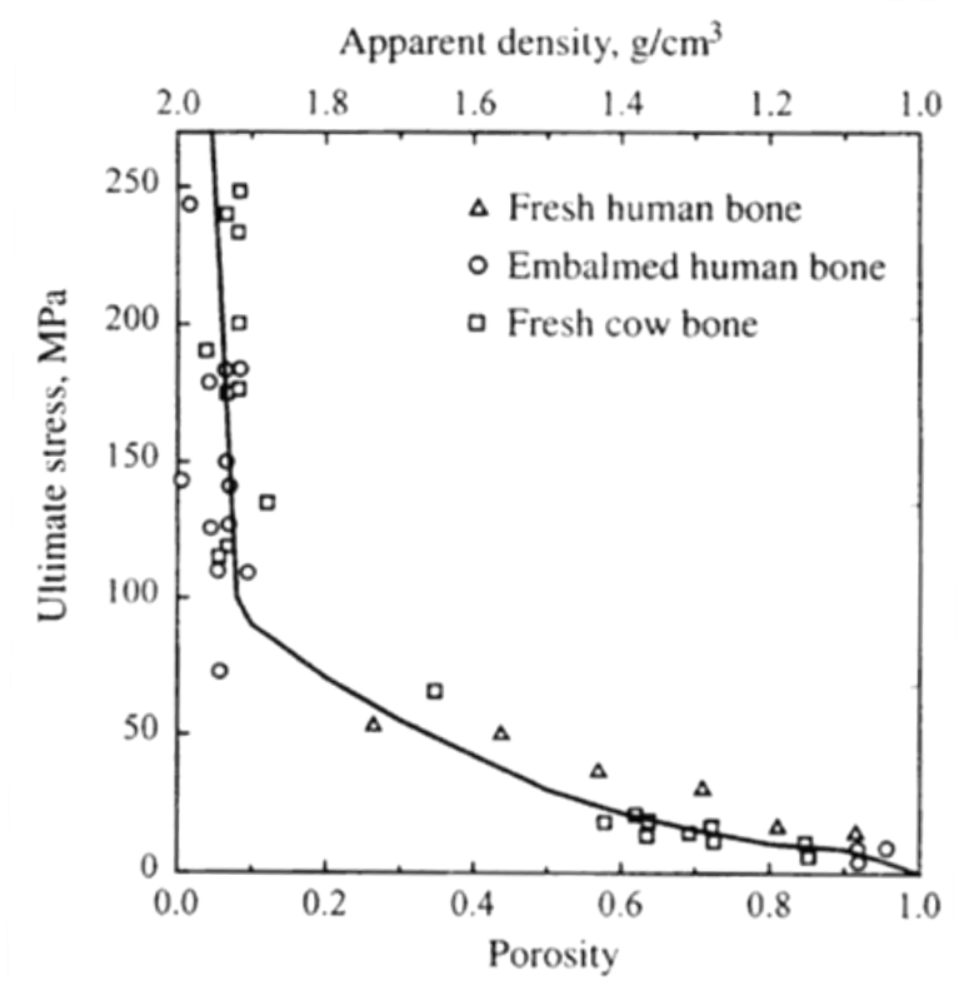

Figure 10. The ultimate compressive stress, porosity, and apparent density relationship for three types of bone [12].

\subsubsection{Bone Fracture and Failure Mechanics}

Bone experiences many large loads on a day to day basis and when the remodeling of bone cannot keep up with forces exerted on the bone, fatigue will occur and mostly likely result in a fracture. The failure modes can either be fast fracture, where the crack propagates rapidly, or fatigue fracture, which is due to repeated loading and stress fractures [11]. Bone undergoes functional adaptation to reduce fracture by having bone structure optimized for strength with respect to weight, trabecular alignment along the principle stress directions, and bone adaptation to the local mechanical environment through activity of bone cells [11]. According to Wolff's law, bone remodels in response to its mechanical environment [11]. Wolff's law is a scientific 
paradigm to which data are routinely fit, intended to explain how bone repairs its self and changes shape due to continual loading [12].

\subsubsection{Quantifying Architectural Features of Bone}

Different techniques can be conducted to quantify the architectural features of bone. It is important to have these methods or techniques so that factures can be prevented. Advancements have occurred which allow measurements of bone properties in noninvasive methods. One method to be utilized to quantify architectural features is a technique that can be conducted noninvasively by photon absorptometry, which uses beams of photon scanning, and is an improved version of DEXA [12]. DEXA is used to quantify the bone mass. Another method includes an ultrasound, which shows promise for analyzing the mechanical properties of trabeculae with the use of refraction [12]. One imaging techniques, called micro and quantitative computed tomography $(\mathrm{CT})$, can capture a 3D representation of a bone sample in a nondestructive way, however for this method a sample of bone has to be removed from the organism to be imaged [16]. This can help identify fractures, micro-fractures, and other pathologic traits that may affect the geometry of the bone [12].

Other methods for quantifying architectural features of bone are more commonly used in research settings or where the previously listed methods are lacking, since they are invasive measurements. Stereology is one example of an invasive measure which analyzes a 3D structure with $2 \mathrm{D}$ [12]. This is done by cutting the sample into a histological section and measuring the volume fraction of the voids in the image [12]. Other techniques include histological methods analyzed with a microscope, which are particularly useful in research settings and are good at looking at porosity. Overlaying grids, in this case, can be used to measure the porosity. One of 
these types of grids is a Merz grid, seen in Figure 11, which consists of 36 points on 6 hemicircular lines. The grid, which is a glass circle with a printed image seen in Figure 11 that is placed in the eyepiece of the microscope, overlays the bone sample and the number of points out of 36 which fall on porous areas are counted [17]. The points on the grid are also used to identify secondary bone and primary bone on each of these points, while the sinusoidal lines are used for analyzing cement lines or secondary bone interfaces. This thesis uses a Merz grid for analysis of bone samples to determine what is occurring in the overall area of the sample.

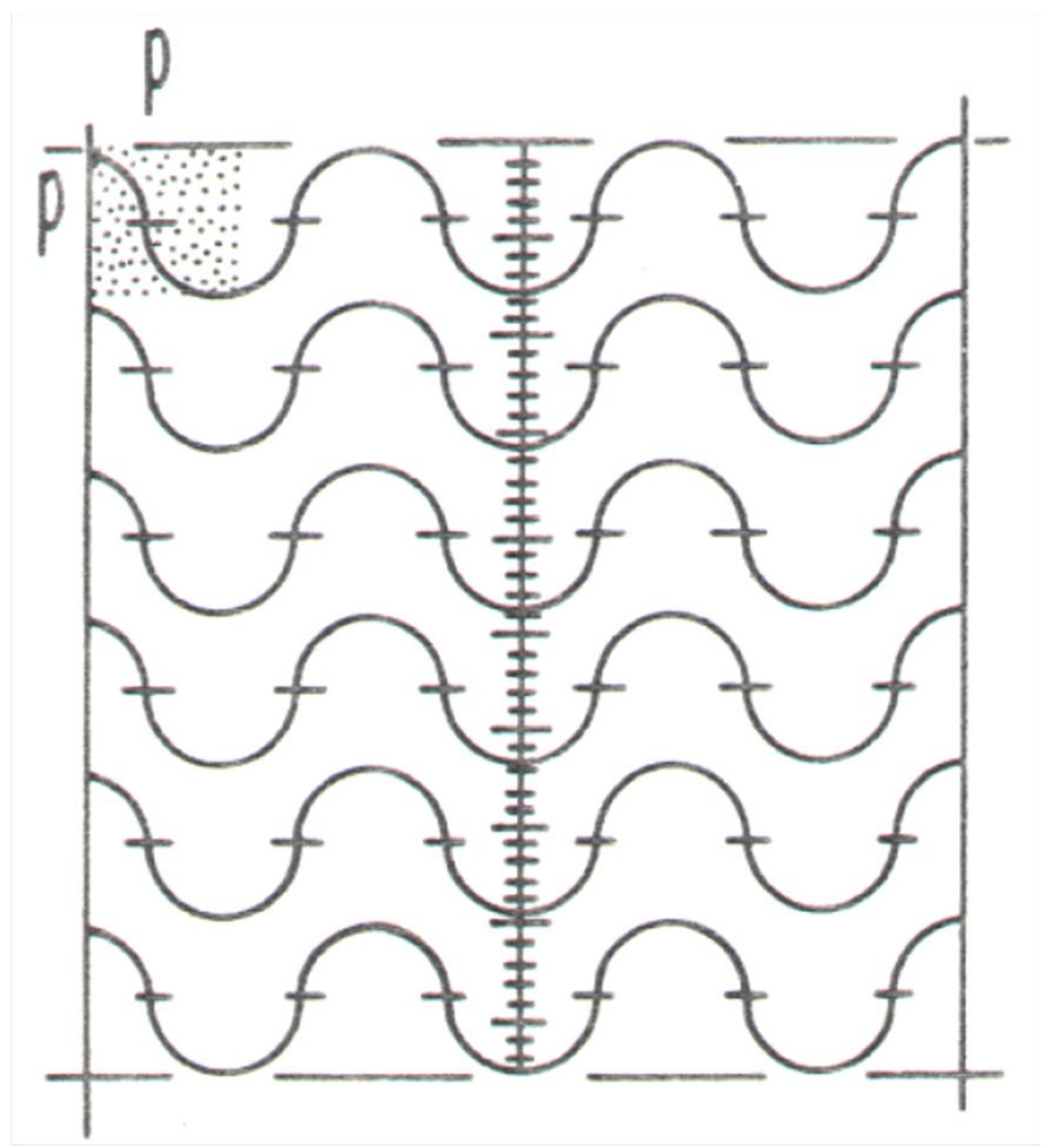

Figure 11. Merz grid used to measure porosity and other bone remodeling characteristics with histology imaging [17]. 


\subsection{Bone Remodeling}

\subsubsection{Bone Modeling vs. Bone Remodeling}

Since bone is a hierarchical, complex material made of many different components which undergo continual loading, it is necessary for bone to remodel its self to maintain the complex structure and strength. Unlike bone modeling, which usually only occurs during growth and in which osteoblasts and osteoclasts work independently of each other, bone remodeling occurs when osteoblasts and osteoclasts are coupled and renew the bone tissue [12]. Modeling also can change the bone's size and shape, while remodeling repairs the bone and in most cases does not affect the size and shape. Although modeling rates reduce greatly in adults, it is a continuous process at particular sites, while remodeling has a distinct beginning and end to its cycles [12]. Bone modeling is necessary during development to create the correct geometry of the skeletal system [12].

The purpose of bone remodeling is to remove old bone and replace it with new bone, which prevents fatigue from occurring because microscopic damage is repaired [12]. Remodeling is done with a combination of osteoblasts and osteoclasts, which together form a basic multicellular unit (BMU) [12]. A BMU is composed of hundreds of osteoblasts and around 10 osteoclasts [12]. They resorb radially outward to take away bone, while they form radially inward and leave space in the center for blood vessels and nerves [12]. Osteonal remodeling replaces about $5 \%$ of human cortical bone each year [12].

\subsubsection{Remodeling Cycle}

BMUs have three main stages in their lifetime which include activation, resorption, and formation (ARF) [12]. Initially to remove bone, osteoclasts form by monocytes fusing together 
from chemical or mechanical signals, called activation. Resorption then takes place where osteoclasts remove bone at a rate of 40 micrometers per day and typically remove a diameter of 200 micrometers [12]. This then allows for formation to occur, initiated by osteoblasts being differentiated from mesenchymal cells, which takes a couple of days [12]. Once the cells are differentiated, the osteoblasts form new bone which is a much slower process than resorption [12]. The time for each stage differs: resorption lasts about 3 weeks, formation lasts about 3 months, and the total remodeling process takes about 4 months $[12,18]$. A sketch of an osteonal BMU can be seen in Figure 12, which displays the stages of resorption, reversal, and refilling. The structure the BMU leaves behind is a secondary osteon [12].

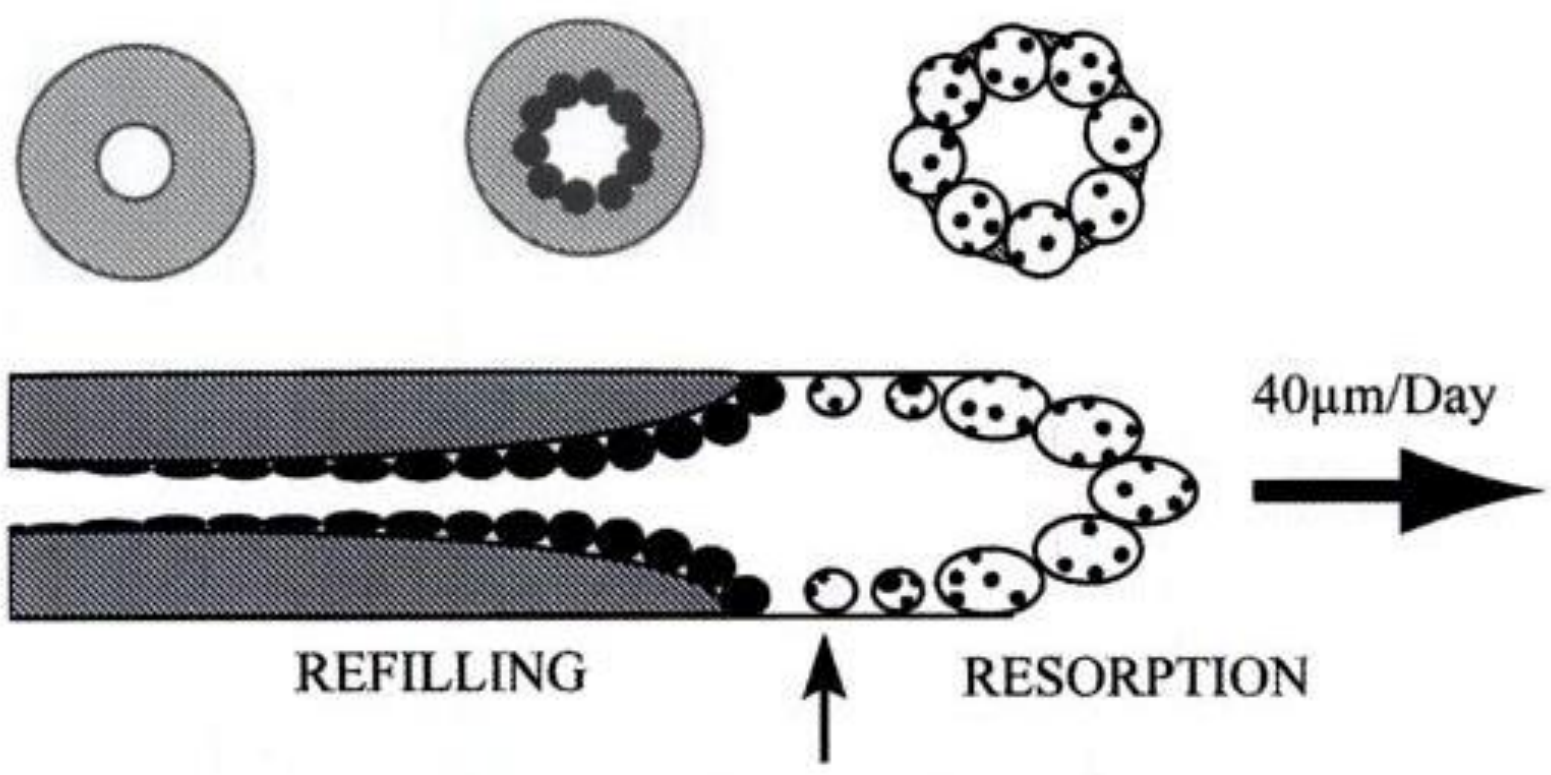
REVERSAL

Figure 12. Sketch of an osteonal BMU. The small black cells on the left are osteoblasts and the larger white cells on the right are osteoclasts [12]. 


\subsubsection{Components of Cycle}

There are 6 more detailed phases for the ARF stages that can describe the life of an osteon; these include activation, resorption, reversal, formation, mineralization, and quiescence [12]. Activation occurs when precursor cells differentiate into osteoclasts, which takes about 3 days [12]. Resorption is when the osteoclasts, which have just been differentiated, start to resorb bone in a longitudinal direction on the cutting cone (200 micrometer diameter, around 300 micrometers in length, and 40 micrometers/day) [12]. There is then a change from osteoclast activity to osteoblast activity, called the reversal phase, which takes a few days. It takes about 30 days for humans to go through the resorption and reversal periods [12].

Regrowth begins to occur in the formation stage of the life of an osteon. It starts off with osteoblasts migrating to the periphery of the vacancy formed by the osteoclasts, where they then begin to refill the vacancy. The average rate that the bone is refilled is 1 to 2 micrometers per day; however the rate for refilling is faster at the beginning of regrowth [12]. Bone is not filled in completely in the osteon; in the center there is a Haversian canal. The Haversian canal is 40 to 50 micrometers in diameter, which will allow blood and nerve signals to be delivered to the bone [12]. This will help provide nutrients during the remodeling process, such as calcium and phosphorus [12]. All together the formation phase takes about 3 months in humans [12].

Mineralization also needs to occur to regrow bone. In this step, minerals are deposited to the collagen fibers. There is a slight delay of about 10 days between formation and mineralization, called mineralization lag time [12]. Mineralization also consists initially of primary mineralization, making up $60 \%$ of mineralization during the first couple days, which is later followed by secondary mineralization [12]. Secondary mineralization occurs over the remaining 6 months at slow rates [12]. This makes it so bone that has just been remodeled has 
different mechanical properties than old bone in the matrix. The final stage is quiescence, which is when the resorption and refilling have been conducted. There are no longer osteoclasts present, and previous osteoblasts either disappear or turn into osteocytes or bone lining cells [12]. Figure 13 displays the location on the BMU where each of the 6 phases of the life of a BMU occur. The completed structure from refilling of a BMU produces secondary osteons, which are the structures which will be analyzed in this study.

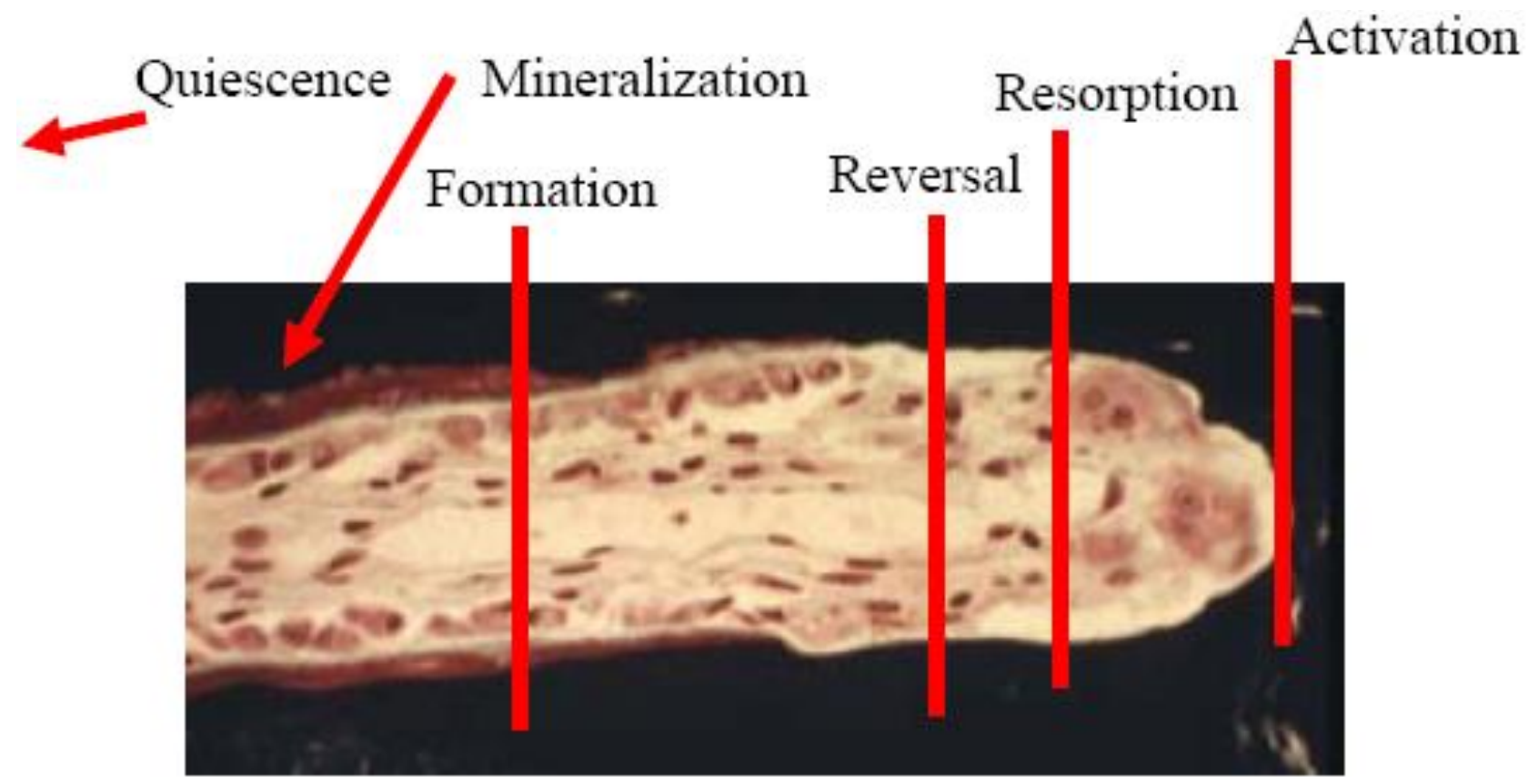

Figure 13. Photomicrograph displaying the regions of the

6 stages of the osteon's lifetime on a BMU [11].

\subsubsection{Characteristics of Remodeled Bone}

Haversian systems, or secondary osteons, have been shown to decrease the strength of the cortical bone [12]. The decrease in strength may be described by the lack of mineralization in newly formed osteons, the increased amount of porosity from Haversian canals, and the increased amounts of plastic deformation [12]. This has been discovered by many different 
animal models and few human models [12]. These experiments have found that Haversian bone shows a decreased compressive and tensile strength compared to primary bone [12].

\subsubsection{Quantifying Remodeled Bone}

One useful method to quantify bone that has been remodeled is to complete histological stains. Researcher, orthopedist, and surgeon, Harold Frost, developed a method which is still currently used with the addition of modern advancements [17]. It labels bone histological samples twice at different time points (usually 7 to 14 days apart) with a tetracycline stain, which can quantify the mineralizing bone and the remodeling rates $[12,17]$. A simplified version of Frost's method is displayed in Figure 14 where BMUs have been labeled twice with a time gap between the two labels. The black, scalloped edged shapes, labeled with an "R" are BMUs in the process of resorption. The circular shapes, with a black dot in the middle (displaying the Haversian canal), labeled with a "C" are osteons that are complete. The shapes labeled "C,S" are also completed osteons, but they have one label on them, meaning that they completed filling in the time between when the first label was delivered and then the second label was delivered. The shaped labeled with an "F" are BMUs that are in the process of filling. The "F,S" label means that the BMU started filling after the first label was delivered and the " $F, D$ " label means that the BMU was filling during the delivery of both labels. $\mathrm{D}_{\mathrm{L}}$, for Frost's method, is the distance between the two labels and is an important parameter when calculating remodeling characteristics, such as the mineral apposition rate. 

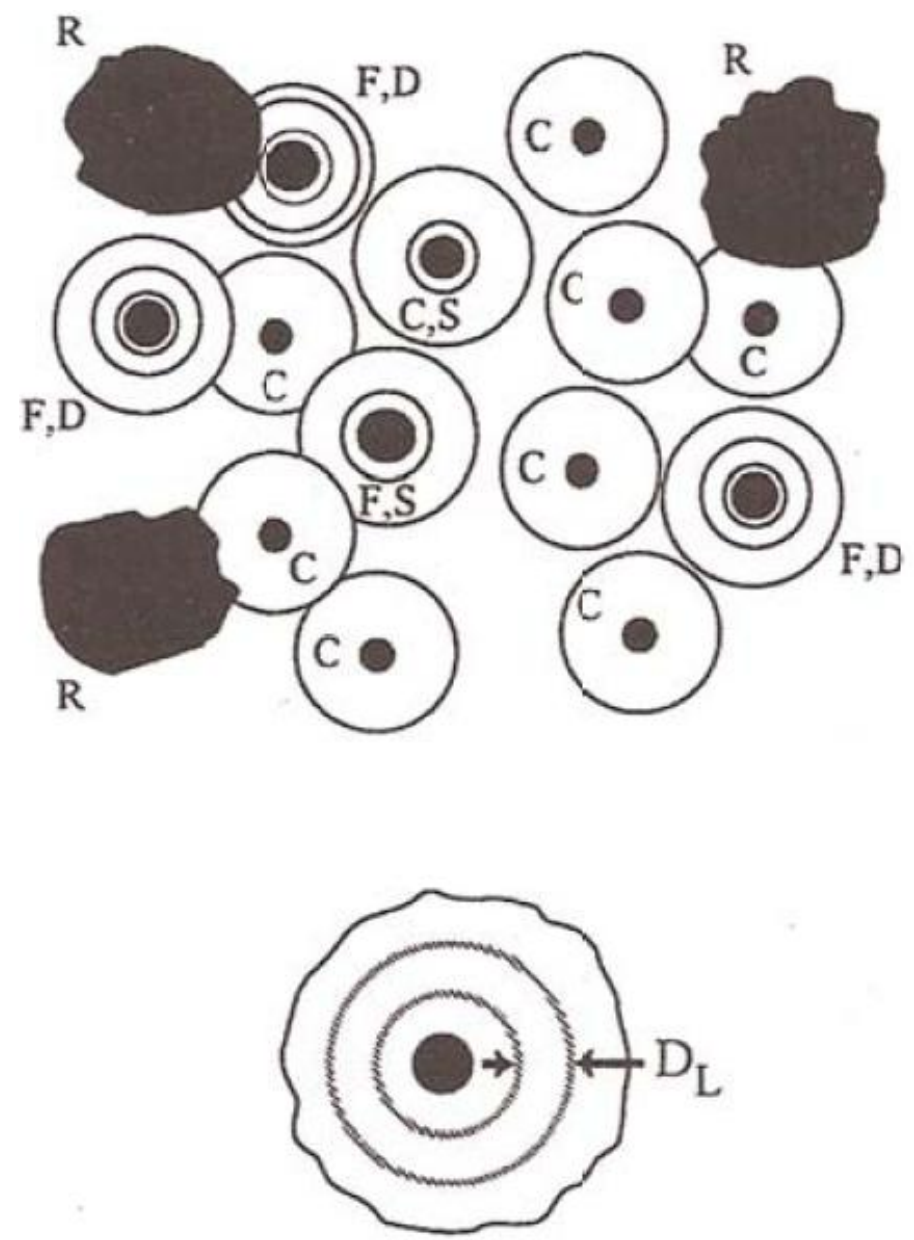

Figure 14. Bone remodeling diagram: (Top) BMUs in a cross section of cortical bone with (R) resorption spaces, (C) completed osteons, (C,S) completed osteons with a single label, (F,S) filling BMUs with a single label, and (F,D) filling BMUs with a double label.(Bottom) An individual BMU in the final stages of filling, with the distance between labels displayed as $\mathrm{D}_{\mathrm{L}}[12]$.

The histological samples of bone used are typically 100 micrometers thick and stained to identify the osteoid mineralized bone in addition to the two labels [17]. The samples are then put onto slides and analyzed with a microscope. The measurements conducted on the samples include calculating the cross sectional area, counting BMUs, identifying resorption spaces by the scalloped surfaces and lack of stains, and calculating the mean perimeter of the resorption spaces 
[17]. The osteoid seams are measured to find the mean thickness and perimeter. In addition the completed osteons are measured to find the mean distance from the cement line to the Haversian canal.

The samples used in this study are microradiographs have not been labeled or stained, they are X-rays, and so other methods need to be used to determine the remodeling characteristics. These methods can look at particular characteristics of bone: such as porosity, number of cement line interfaces, number of secondary osteons, the amount of remodeled bone, densitometry, etc. Counting secondary osteons, for example, can be an alternative way to quantify the rates of bone turnover [12].

\subsubsection{Encroached Secondary Osteons}

Another possible informative measurement is to quantify the amount of encroached, or partial, secondary osteons per area. As more remodeling occurs, osteons begin to overlap each other so encroached secondary osteons will become present in histological samples. Also as new secondary osteons are formed, or unencroached secondary osteons, the osteoblasts may add bone in locations that used to be an older osteon's Haversian canal. Although, the presence of Haversian canals and other porous structures in remodeled bone supports the idea that there is an age related increase in porosity from incomplete refilling [12]. Analyzing encroached secondary osteons has mostly been done to determine the age of an individual when deceased [12]. Osteoporosis and other diseases could also be analyzed by measuring the encroached secondary osteons in samples of cortical bone. Encroached secondary osteons can also be useful when determining the rates of activation and bone turnover [12]. A microradiograph displaying partial 
(encroached) secondary osteons, as well as whole (unencroached) secondary osteons and interstitial regions are shown in Figure 15.

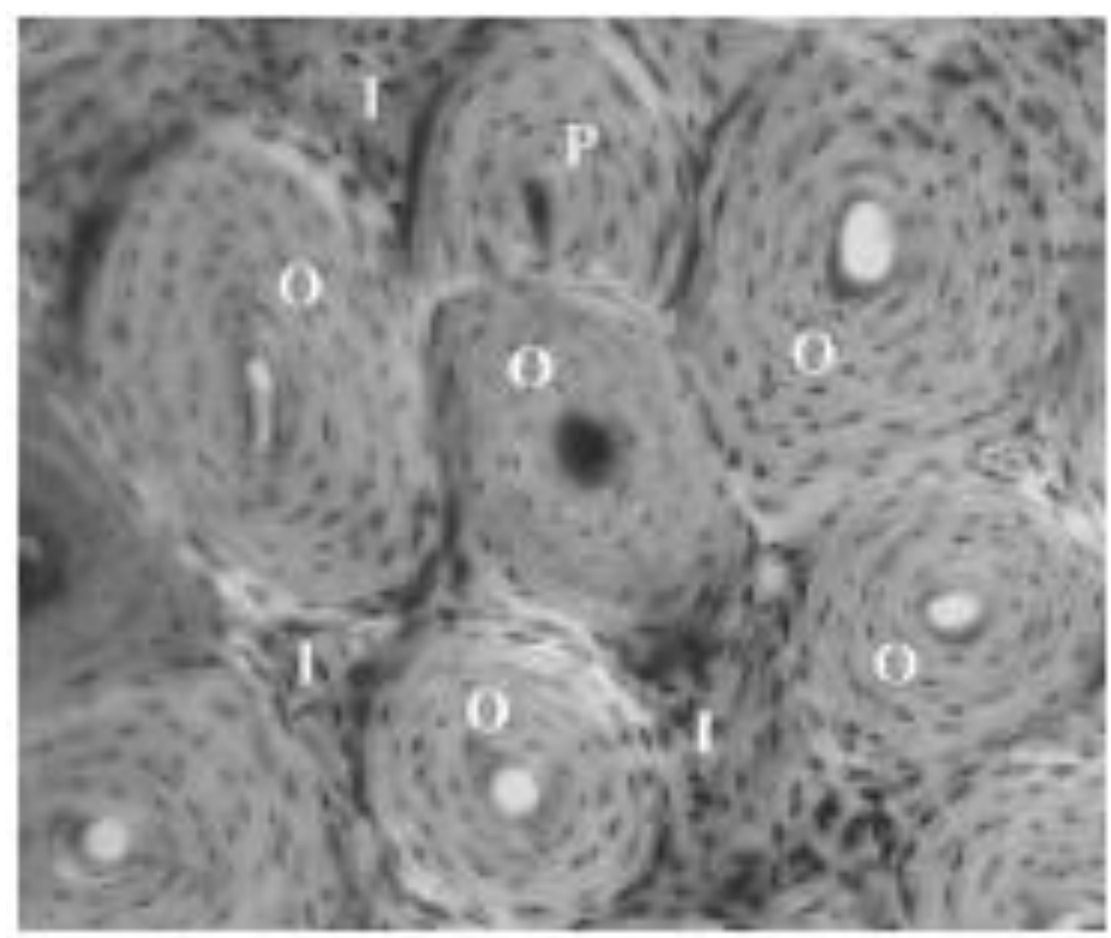

Figure 15. Microradiograph of equine bone: partial osteons $(\mathrm{P})$, whole osteons (O), and intersitial regions (I) [19].

Analyzing encroached secondary osteons, also referred to as osteon fragments, have mainly been used as a static histomorphomety method to determine the age of death of skeletal remains that anthropologists discover [12]. However, little to no research has been done to utilize encroached secondary osteons to further understand remodeling in animal models and to determine if the remodeling is occurring frequently in the same places. In order to make histomorphometry measurements, an exact definition for complete and encroached secondary osteons is needed so that the correct structures can be identified, however, previous researchers have defined encroached secondary osteons and complete secondary osteons in a variety of ways. Kerley in 1965 published an article about determining age based on the microscopic 
changes in cortical bone. He defined complete secondary osteons as structures with $80 \%$ or more of their osteonal area intact and which surround a complete Haversian canal [20]. A secondary osteon fragment, he defined, as having less than $80 \%$ of the osteonal area intact [20]. A histological image of human bone with an osteon fragment labeled, according to Kerley's method, can be seen in Figure 16.

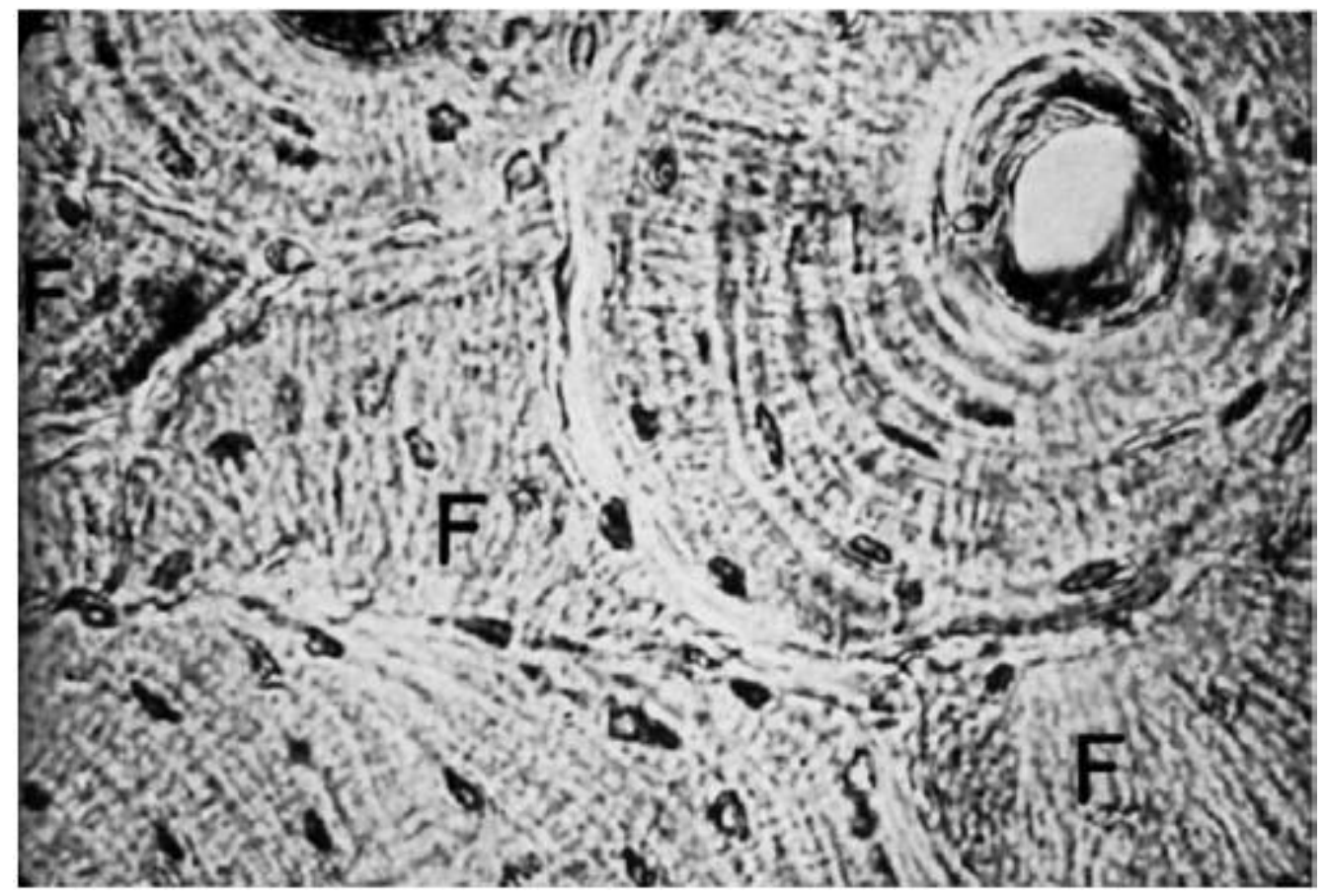

Figure 16. A histological slide with osteon fragments (F) [20].

In 1983, Stout was working on analyzing ancient skeletal remains where he defined complete secondary osteons based on the Haversian canal [21]. His definition defined complete secondary osteons as having $90 \%$ of the Haversian canal perimeter intact and secondary osteon fragments as having less than $90 \%$ of the Haversian canal perimeter intact. Burr later published research comparing histology of ancient remains compared to modern samples where he defined a complete secondary osteon as a structure having $100 \%$ of the Haversian canal perimeter intact and a secondary osteon fragment as a structure with any of the Haversian canal perimeter 
remodeled away [22]. Ericksen, while determining the age of death based on histology of femurs, defined secondary osteon fragments as a remnant of former osteons that can range from tiny slivers to fully-sized "dead" osteons whose Haversian canals clearly show Howships lacunae [23]. A schematic of Ericksen's definition of secondary osteon fragments and complete secondary osteons can be seen in Figure 17. In 2008, Robling and Stout, working on age estimation histology methods, defined secondary osteon fragments based off of Stout's definition in 1983. They created a measurement called N.On.Fg., or the number of fragmentary secondary osteons, which was seen as secondary osteons which have $10 \%$ or more of the perimeter of the Haversian canal remodeled by subsequent generation of secondary osteons [24]. The fragmentary secondary osteons also include osteons with remnants of preexisting secondary osteons that no longer have Haversian canals [24]. 


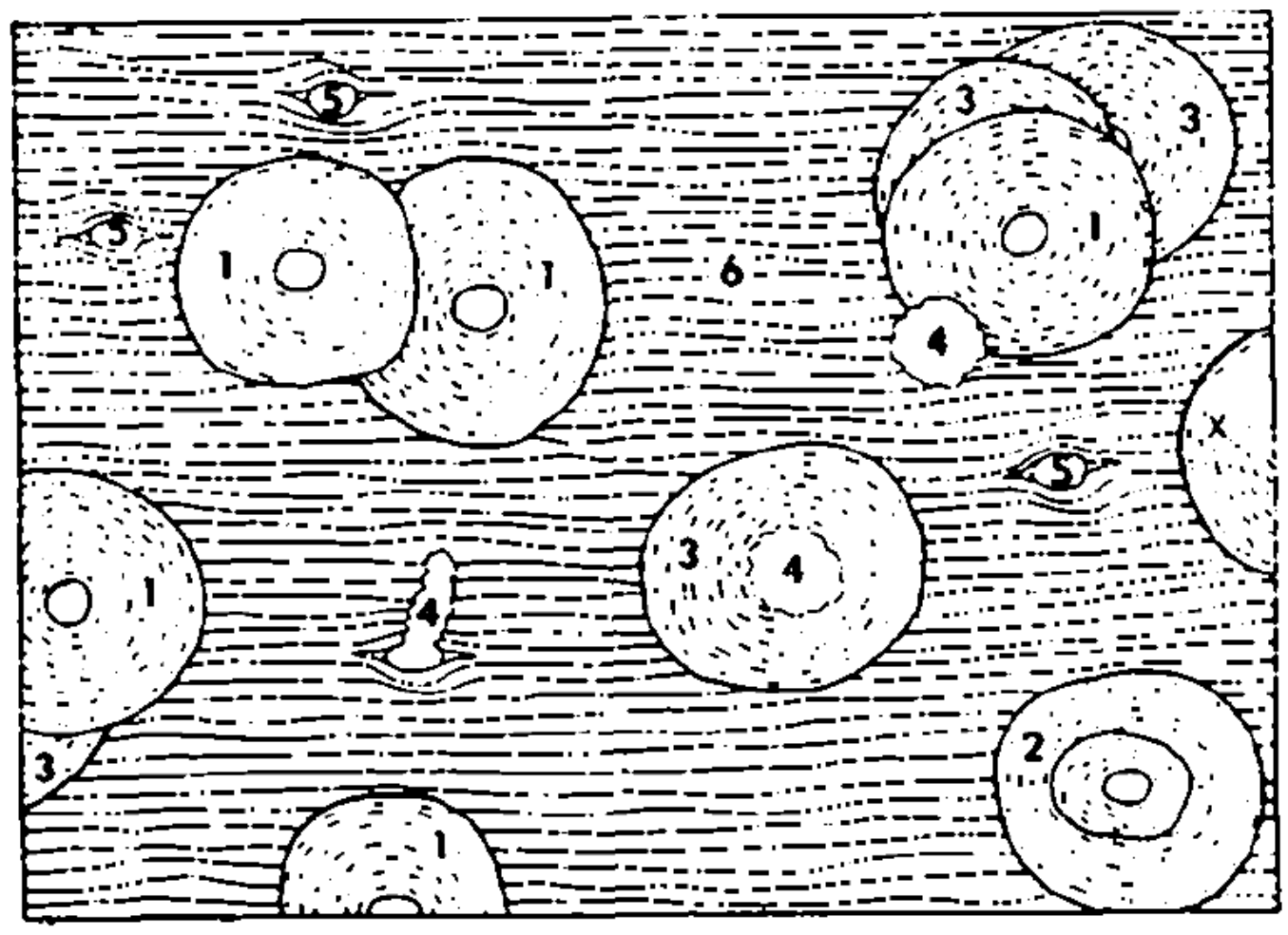

Figure 17. A diagram of the cortical bone microstructure with (1) complete secondary osteons,

(2) secondary osteons within secondary osteons, (3) secondary osteon fragments, (4) resorption spaces, (5) non-Haversian canals, and (6) primary bone [23].

Since researchers have defined secondary osteon fragments as a percentage of complete secondary osteons, it is hard to visually determine what percentage of a secondary osteon is encroached because the complete secondary osteon size is unknown. This can lead to inaccurate and variable data. This study defines its own definition of an incomplete secondary osteon based on the previous literature. A summary of previous definitions and the definition in this study of complete secondary osteons compared to incomplete secondary osteons can be seen in table 1 . 
Table 1. Table displaying definitions of complete secondary

osteons compared to incomplete secondary osteons.

\begin{tabular}{|c|c|c|}
\hline Reference & Complete Secondary Osteons & Incomplete Secondary Osteons \\
\hline Kerley 1965 & $\begin{array}{l}80 \% \text { or more of the osteonal area } \\
\text { intact }\end{array}$ & $\begin{array}{c}\text { Less than } 80 \% \text { of the osteonal area } \\
\text { intact }\end{array}$ \\
\hline Stout 1983 & $\begin{array}{c}\text { At least } 90 \% \text { of the Haversian } \\
\text { canal perimeter intact }\end{array}$ & $\begin{array}{c}\text { Less than } 90 \% \text { of the Haversian canal } \\
\text { perimeter intact }\end{array}$ \\
\hline Burr 1990 & $\begin{array}{l}\text { Secondary osteons with whole } \\
\text { Haversian canals }\end{array}$ & $\begin{array}{c}\text { Any of the Haversian canal perimeter } \\
\text { remodeled away }\end{array}$ \\
\hline Ericksen 1991 & $\begin{array}{c}\text { Secondary osteons with whole } \\
\text { Haversian canals that do not show } \\
\text { Howships lacunae }\end{array}$ & $\begin{array}{l}\text { Remnants of former osteons ranging } \\
\text { from tiny slivers to full sized "dead" } \\
\text { osteons showing Howships lacunae }\end{array}$ \\
\hline $\begin{array}{l}\text { Robling and } \\
\text { Stout } 2008\end{array}$ & $\begin{array}{l}\text { At least } 90 \% \text { of the Haversian } \\
\text { canal perimeter intact }\end{array}$ & $\begin{array}{l}\text { Less than } 90 \% \text { of the Haversian canal } \\
\text { perimeter intact and remnants with no } \\
\text { Haversian canal }\end{array}$ \\
\hline This study & $100 \%$ of the osteonal area intact & $\begin{array}{l}\text { Any of the osteonal area remodeled } \\
\text { away }\end{array}$ \\
\hline
\end{tabular}

\subsection{Previous Animal Models}

When studying bone remodeling animal models are commonly used. Bone is complex, so creating studies where animals can be sacrificed to enable a histological study of their bones is the common approach. Research begins on smaller animals and then moves to larger animals with specific similarities to humans.

For initial research on bone remodeling, testing on rodents has proved to be effective and less expensive than larger animal models. Small animal models are also good for preliminary research since they usually develop faster than larger animals, making the time period of the study shorter. This however is not an ideal model since rodents undergo a lot less remodeling compared to humans. One preliminary research study found that mice with an ovariectomy 
surgery showed a significant effect on remodeling [25]. An ovariectomy surgery (OVX) is a surgery that is conducted to remove the ovaries of the animal, which is compared to a sham surgery, or the control [25]. The mice were then administered bisphosphonates which showed remodeling rates lowering with the treatment of the bisphosphonates [25]. Another study looked at rats with an ovariectomy to also study the effect of bisphosphonates, which showed the effect of estrogen deficiency [26]. Rats however have considerably less Haversian remodeling than larger, longer lived species so it is extremely difficult and not very valuable to compare the results to humans [27].

Other small animals, such as rabbits, have also been used to study osteoporosis and bone remodeling, which have shown informative results. Rabbits have shown to be a better model for humans, compared to mice and rats, since they also undergo Haversian remodeling [28]. Rabbits typically are used for bone studies that look at bone ingrowth to implants [27]. Although rabbits have demonstrated to be a good small animal model while looking at the effect of osteoporosis on the skeletal system, larger animal models are necessary to compare results to humans.

Multiple large animal models have been attempted for studying osteoporosis and bone remodeling. One unsuccessful animal model for studying osteoporosis used dogs. This is because dogs with an ovariectomy, measured decrease in estrogen levels, and a sedentary lifestyle, showed limited change in bone mass and no increased fracture risk compared to dogs with normal levels of estrogen [27]. Dogs also have a semiannual reproductive cycle compared to humans who have a reproductive cycle 12 to 13 times per year [27]. Another downside of the dog animal model is that most domestic dogs have been spaded, or ovariectomized, to prevent impregnation, which has shown minor to no loss in bone and no change in fracture risk [27]. Another large animal model tried was with nonhuman primates. Primates ideally would be a 
great model when studying bone remodeling and osteoporosis, however the huge cost and time involved with studying primates is not practical for most research studies [27].

Sheep models have shown to be one of the most effective large animal models for studying bone remodeling and osteoporosis. Sheep bone has the most similar secondary osteon size to human bone compared to other animal models [27]. Young sheep bone initially consists of primary bone that is both lamellar and woven bone, and as it ages it is gradually replaced with remodeled secondary bone [27, 29]. The plexiform and Haversian remodeling bone of sheep can be seen in Figure 18. Sheep also have shown quantifiable similarities to human females for hormonal profiles [29]. Multiple studies have looked at sheep with ovariectomies and found significant decreases in bone mineral density [29]. 

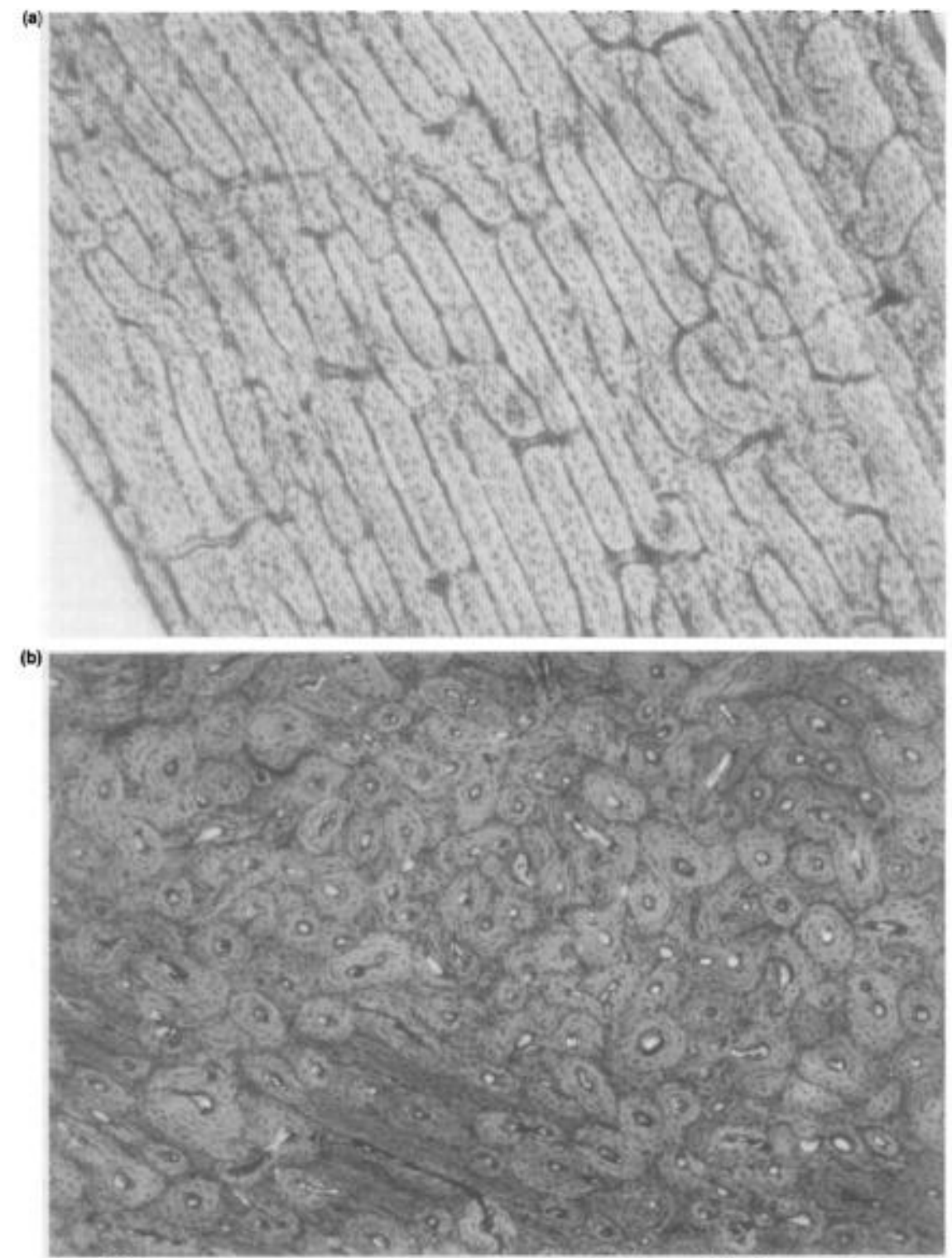

Figure 18. Histology samples at 25x magnification of (a) 3 year old ewe plexiform bone, and (b) 8 year old ewe Haversian remodeling of the caudal femoral cortext [27].

One disadvantage however for using sheep as a model for postmenopausal osteoporosis is that female sheep have estral cycles, rather than menstrual cycles like humans [30]. They are seasonally polyestrous, usually in the autumn months, with estrous cycle lengths lasting on average about 17 days. Polyestrous cycles continue until the sheep has bred or the ewe returns to anestrus [30]. Fortunately, during the anestrus period, the levels of estrogen in the ewe are 
relatively high [30]. Sheep also have a slight difference in their gastrointestinal system because they have rumen microflora, a part of the esophagus and stomach allowing the animal to be able to digest certain plant based foods. The presence of the rumen microflora in the esophagus and stomach complex may alter the effect of drugs delivered to the sheep compared to the effects of drugs on humans, who have microflora in the cecum and larger intestine [29].

Despite a few drawbacks, the ovariectomized sheep has many possible strong points so it was the selected model to use in this study to try and simulate postmenopausal osteoporosis. Characteristics which have shown to effect remodeling rates in sheep also include age, region of the bone, and the season the samples are taken from. These factors are taken into account for this thesis so the effects of the ovariectomy can be normalized. Ovariectomized sheep will be compared to sheep that have also undergone surgery and had their ovaries handled, but not removed. The term used for the mock surgery in this study is a sham surgery. Analyzing remodeling factors based on the effect of the OVX surgery compared to sham surgery will help determine if this model is suitable studying postmenopausal osteoporosis effects on bone.

\subsection{Objectives}

The purpose of this thesis is to further quantify adult ovine bone for new remodeling characteristics to obtain a better understanding of how remodeling is occurring. Previous students' theses have analyzed the same samples for basic bone remodeling histology measurements including: the percent of tissue remodeled, the percent of material remodeled, mean secondary osteonal radii, the ratio of bone volume to tissue volume, cement line interfaces as a function of tissue volume, and cement line interfaces as a function of material volume. The results showed that these remodeling parameters had some significant seasonal, anatomical, and 
treatment differences $[31,32]$. However, most of the results showed no particular increase in the amount of remodeled area for the ovariectomized sheep compared to the sham sheep, even though an ovariectomy is believed to cause a burst of remodeling in bone due to the decreased levels in estrogen [32]. One explanation for these results could be that lots of remodeling occurred from the ovariectomy, but only in places where there has already been remodeling. If this is the case, then the ovariectomized ovine bone and the control ovine bone would have the same amount and proportion of secondary osteons, such as unencroached secondary osteons, but the ovariectomized ovine bone would also have more secondary osteons that have been remodeled away, or encroached, by subsequent generations of secondary osteons. The alternative hypothesis is that the ovariectomy surgery has no effect on the ewe bone, which would result in the sham and ovariectomized bone having the same amount and proportion of secondary osteons, encroached secondary osteons, and unencroached secondary osteons. This thesis examines the hypothesis by defining osteon encroachment, generating and completing a method to quantify osteon encroachment, and analyzing the collected data to determine if this is true.

The presence of encroached secondary osteons will be analyzed for sections of bone from 12-month OVX and control sheep histology samples from the summer sacrifice season. The bone samples will be taken from the radius and ulna of the left leg of the sheep and the compact bone will be analyzed. Since previous students have determined that the region the bone specimen is taken from has a significant effect on the amount of bone remodeling [31,32], two different sectors of the bone will be measured; one on the compressive side and one on the tensile side. These results will be analyzed to determine if there are significant effects from the type of surgery the sheep underwent and the sector that the bone specimen is from, as well as looking at the effect of the interaction between the sector and surgery. These finding will better explain the 
mechanism of how ovariectomy surgery affects ovine bone remodeling, if there is an effect, and if this is different when the bone is in tension or compression. The results will also help determine if the ovariectomized sheep is a suitable model to use to study post-menopausal osteoporosis. 


\section{METHODS}

\subsection{Animal Preparation}

Animal specimens for this study were obtained from Colorado State University, Fort Collins where the animals were also used for a larger experiment. 112 Columbia-Rambouillet cross ewes that were skeletally mature and 5 years in age or older were used with approval of the Animal Care and Use Committee. The living conditions for the ewes during the experiment included a diet of a grass-alfalfa hay mixture and housing of dry lots at an altitude of 1500 meters and 41 degrees north latitude. The 112 ewes were then evenly divided into 4 groups of 28 , one group for each season (summer, autumn, winter, and spring). The group of 28 for each season was then divided into 2 groups of 14 , one group for the control and one group for the treatment. The control group underwent a sham surgery and the treatment group underwent a surgery to remove the ewe's ovaries, or an ovariectomy (OVX) surgery.

The surgeries were performed at Colorado State University at the large animal surgery facility for the College of Veterinary Medicine and Biomedical Sciences. During both types of surgeries the ewes were anesthetized. During the control surgery the ovaries were identified, handled, but not removed and during the OVX surgery the ovaries were identified and removed. The season the surgery was conducted in correlated to the seasonal group the ewe was placed in. All of the summer surgeries occurred in August, all of the autumn surgeries occurred in November, all of the winter surgeries occurred in February, and all of the spring surgeries occurred in May. Each of the treatment and control groups for each season were also divided again into 2 groups: one group of 7 where the ewes were scarified 3 months postoperatively and one group of 7 where the ewes were sacrificed 12 months postoperatively. A control ewe from the autumn 12 month group died prematurely, so that specimen was put into the 3 month group 
for autumn. For this study, the 12 month summer ewe specimens for both the control and OVX groups were used.

\subsection{Specimen Preparation}

At Colorado State University, the radius and ulna were removed from both the right and left side of the ewe. The radius and ulna were wrapped in paper towels, soaked in saline, sealed in plastic bags, kept at $-20^{\circ} \mathrm{C}$, and shipped to Henry Ford Hospital in Detroit, Michigan to be prepared for analysis. A band saw (Model 5212, Hobart Corporation, Troy, Ohio) was used to remove the center $50 \mathrm{~mm}$ of the diaphysis. An Exakt cutting-grinding system (Exakt Coropation, Oklahoma City, Oklahoma) was then used to divide the radius into 6 sectors based on anatomical location. These anatomical sectors included the following groups: cranial, caudal, craniolateral, craniomedial, caudomedial, and caudolateral (Figure 19). For this study, only the cranial and caudal anatomical sectors were analyzed.

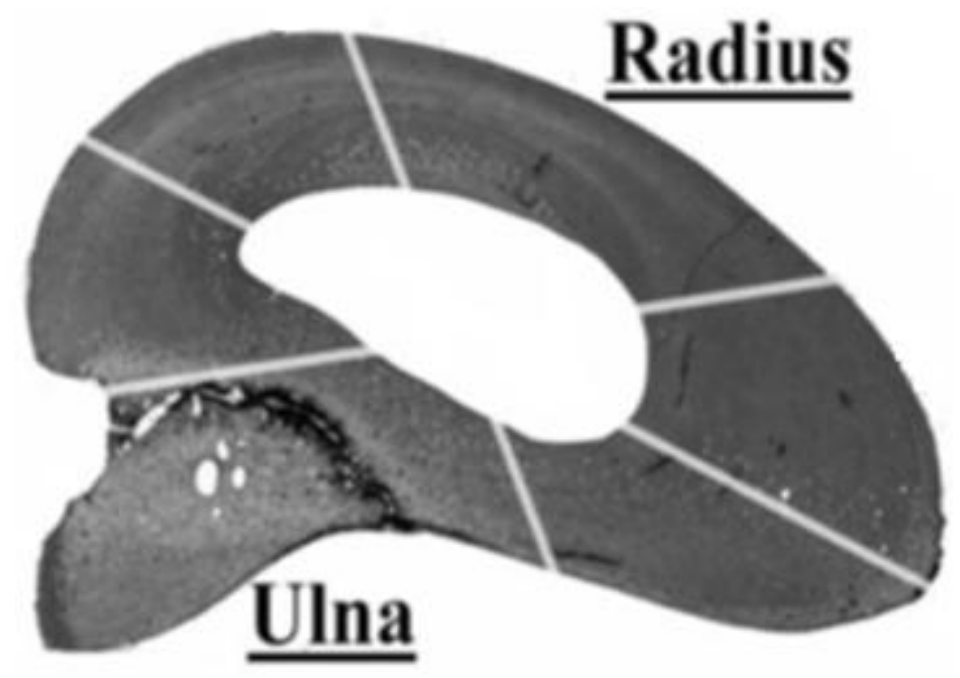

Figure 19. Radial-Ulnar approximate anatomy divided into 6 anatomical sectors. The top left sector is the lateral aspect and the top right is the cranial aspect [31]. 
The pieces of bone were made into 1.75 by 1.75 by $19 \mathrm{~mm}$ cortical beams for each of the anatomical sectors. In another study, dynamic mechanical testing of the left radii and ulnae were preformed. From these left radii and ulnae cortical beams, $150 \mu \mathrm{m}$ sections were cut out of the center. The distal ends of the cortical beams were used to determine the density with a drying and ashing method and the proximal ends were frozen to be further examined in future studies.

The $150 \mu \mathrm{m}$ sections were made into microradiographs. Before producing the microradiographs, the specimens were hand ground down to a thickness of $100 \mu \mathrm{m}$. Fine grit sand paper was used for the grinding. 2506AGHD 2.5X2.5X0.060 High Definition Photo Emulsion Plates and a HB Cabinet Faxitron (HTA Enterprises, Microtome Technology Product, San Jose, California) were used to create the microradiographs, which were taken at $25 \mathrm{kV}$ at $3 \mathrm{~mA}$ for 20 minutes. 6 anatomical sectors for 2 ewes were placed on each microradiograph along with an aluminum foil step wedge in the center of the microradiograph (Figure 20). The Reynolds Aluminum Foil step wedge was used in previous studies to assist in quantifying the microdensitometric characteristics of the specimens. 


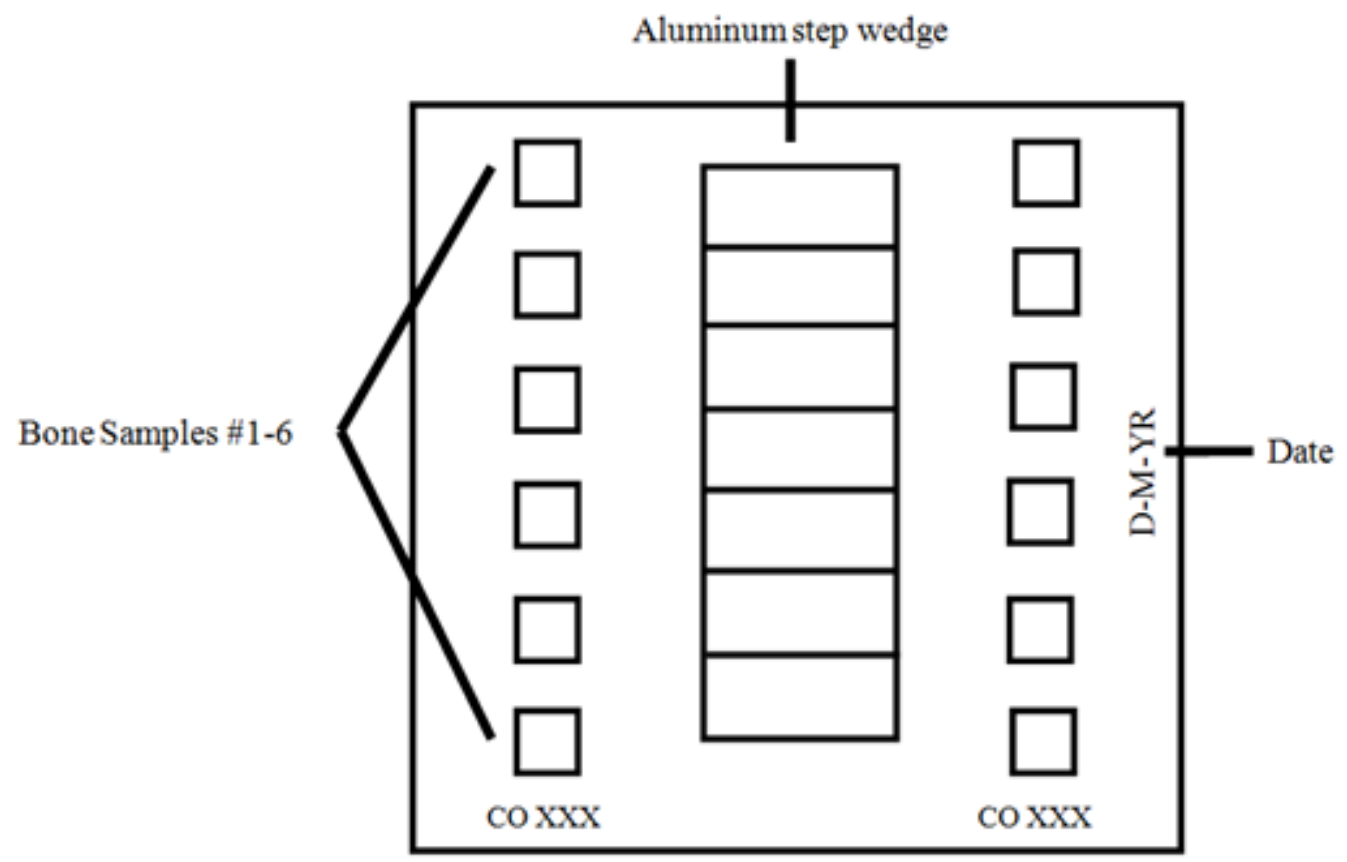

Figure 20. Microradiograph layout diagram [32].

\subsection{Specimen Analysis}

The microradiographs were then sent from Henry Ford Hospital to California Polytechnic State University, San Luis Obispo to be analyzed for multiple measurements. Previous studies have measured various histomorphometric parameters and the densitometry of these specimens. Prior histomorphometric measurements included porosity, number of secondary osteons, number of cement line interfaces, and the amount of remodeled bone. This was used as a method to quantify how much remodeling was occurring in the ewe, or ovine bone. Densitometry measurements were conducted as a method to quantify the density of the ovine bone. This thesis uses histomorphometric methods that were developed to further understand how the remodeling is happening, in particular by looking at unencroached secondary osteons and encroached secondary osteons. 


\subsubsection{Histomorphometry}

An Olympus BX-41 microscope was used with the 10X objective under white light to make the histomorphometry measurements. The cortical beams that were analyzed had measurements taken from 4 quadrants to improve the precision of the measurements. The measurements from those 4 quadrants were then averaged. A Merz grid was used to define the area of the quadrants and to assist in making measurements for both the point count method and the osteon count method.

For each specimen, the Merz grid was aligned so that the edges of the grid's top left corner matched up to the specimen's top left corner and this was considered the first quadrant (Figure 21). After measurements were conducted, the grid was moved to the right so that the grid's top right corner aligned with the specimen's top right corner and this was considered the second quadrant. The Merz grid was then moved downward so that the bottom right corner of the grid aligned with the bottom right corner of the specimen and this was the third quadrant. Lastly, the Merz grid was then moved to the left so that the bottom left corner of the grid and the specimen were aligned and this was the fourth quadrant. If there was not tissue in the entire field of view for a given quadrant, due to uneven edges or part of the bone being broken off, the quadrant was excluded from the average for that specimen for both the point count measurements and the osteon count measurements. This is because the results would be skewed since there would be less than 36 points for the point count method and a smaller area than the normal field of view for the osteon count method. 


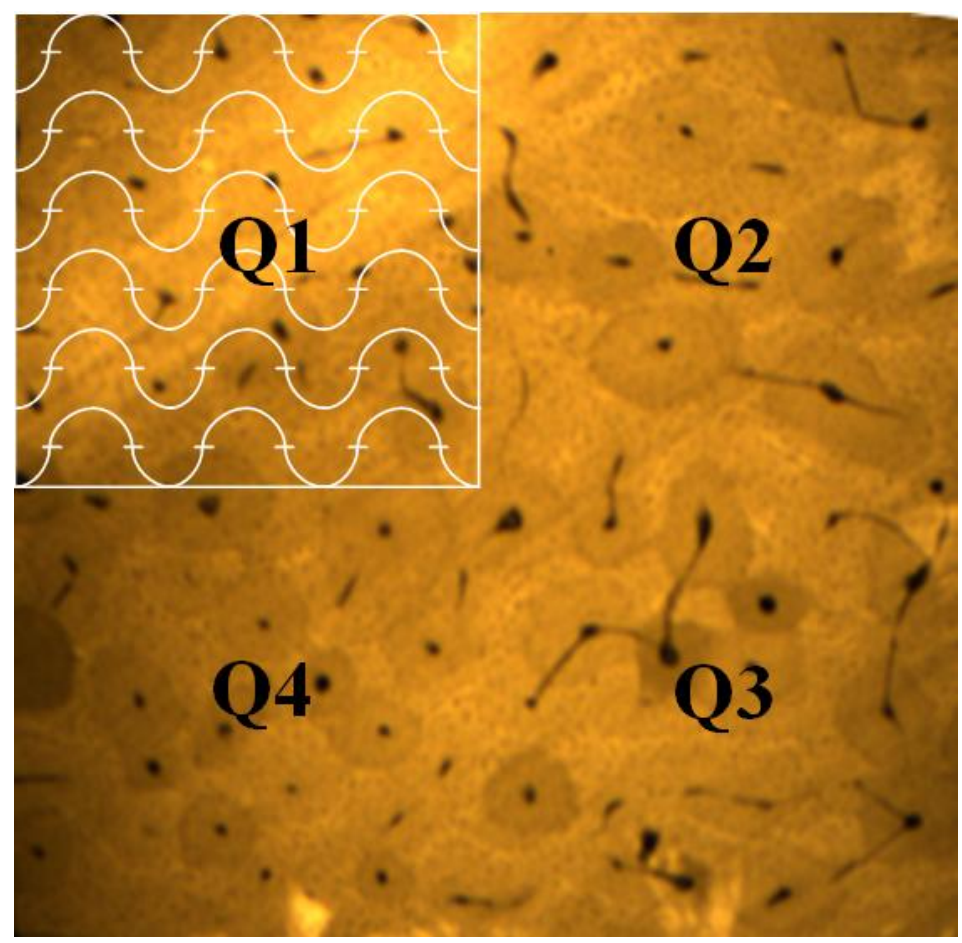

Figure 21. Merz grid aligned in the first quadrant.

\subsubsection{Repeatability}

In order to ensure that the data was repeatable, measurements for the sector 5 samples were measured three separate times. The raw data was classified as a measurement round 1,2 , or 3. This data was then put into SigmaPlot 12.3 (Systat Software, Inc.) and a one way repeated measures ANOVA was conducted. The ANOVA model evaluated the effect of the measurement round (1, 2, and 3) for each of the measurements. The post-hoc Fisher Least Significant Difference (LSD) test was conducted in this analysis to determine the significance between each measurement round. P-values less than 0.05 were considered significant, meaning that the results were not repeatable. 


\subsubsection{Point Count Measurements}

The first collection of measurements that were taken from each quadrant of each specimen used the 36 points on the Merz grid. Each point on the grid was classified as porosity, primary bone, secondary unencroached bone, or secondary encroached bone based on what the point on the grid aligned with on the specimen. For each quadrant, the sum of all of the porosity, primary bone, secondary unencroached bone, and secondary encroached bone points must add up to 36 .

A point was classified as porosity if it fell on a non-bone material, such as Haversian canals, Volkmann's canals, or remodeling cavities (Figure 22). A point was classified as primary bone if it was original bone laid down (Figure 23). Primary bone has lamella that is parallel to the surface of the bone, but primary bone can be more easily identified as points that are not porosity or secondary bone. Secondary bone points were identified as circular structures centered on a Haversian canal with a cement line, circular lamella, and an osteocyte lacunar pattern. To classify a point as unencroached secondary bone it must have an intact whole secondary bone structure which has not been remodeled away at all by other secondary bone structures or resorption cavities (Figure 24). Also for unencroached secondary bone, a whole intact Haversian canal must be present and a clear cement line which is unobstructed. To classify points as encroached secondary bone, the point should fall on secondary bone structures that appear to have any section remodeled away by subsequent generations of osteons or resorption cavities

(Figure 25 and Figure 26). Also part, all, or none of the Haversian canal can be remodeled away and the cement line is not complete and can show concave sections. 


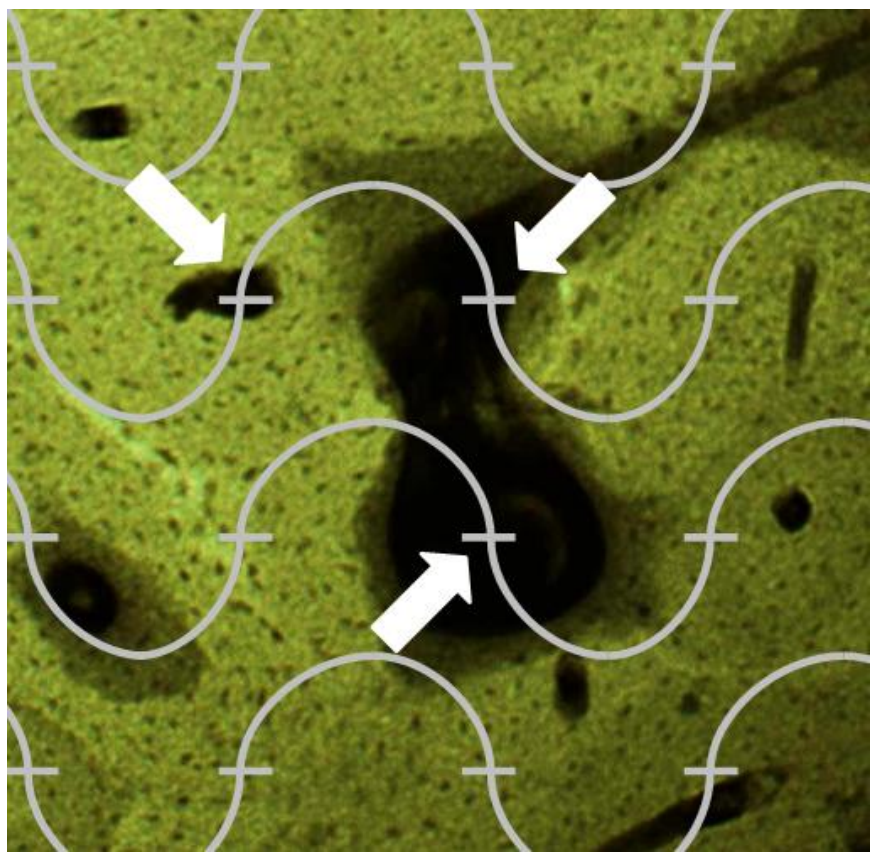

Figure 22. Merz grid points located on porosity.

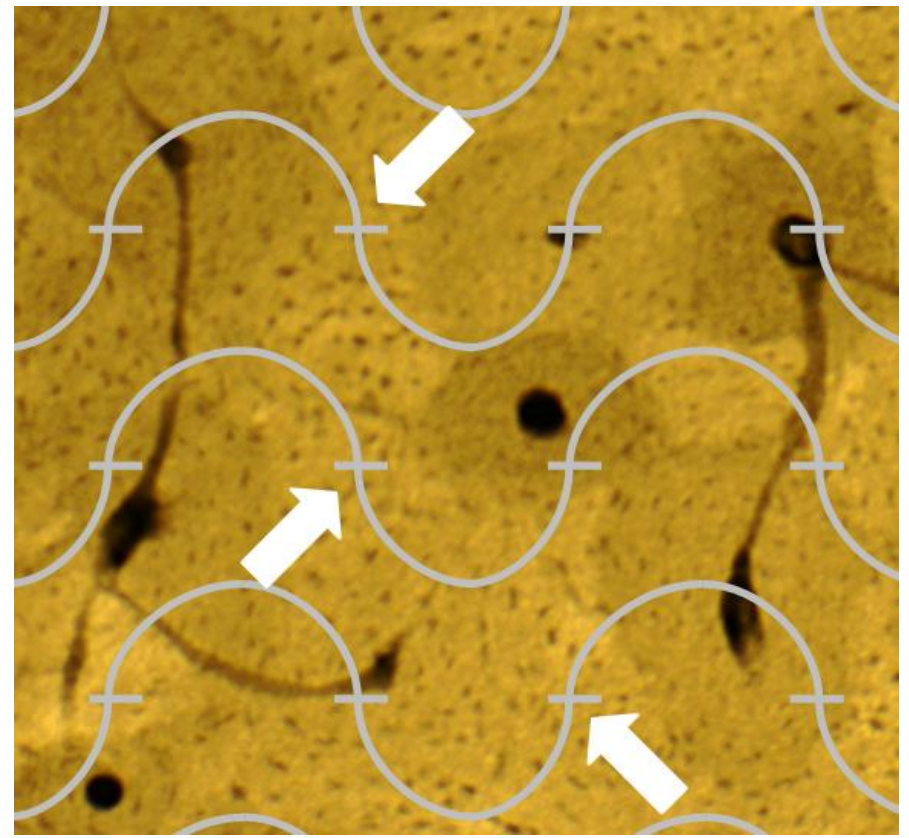

Figure 23. Merz grid points located on primary bone. 


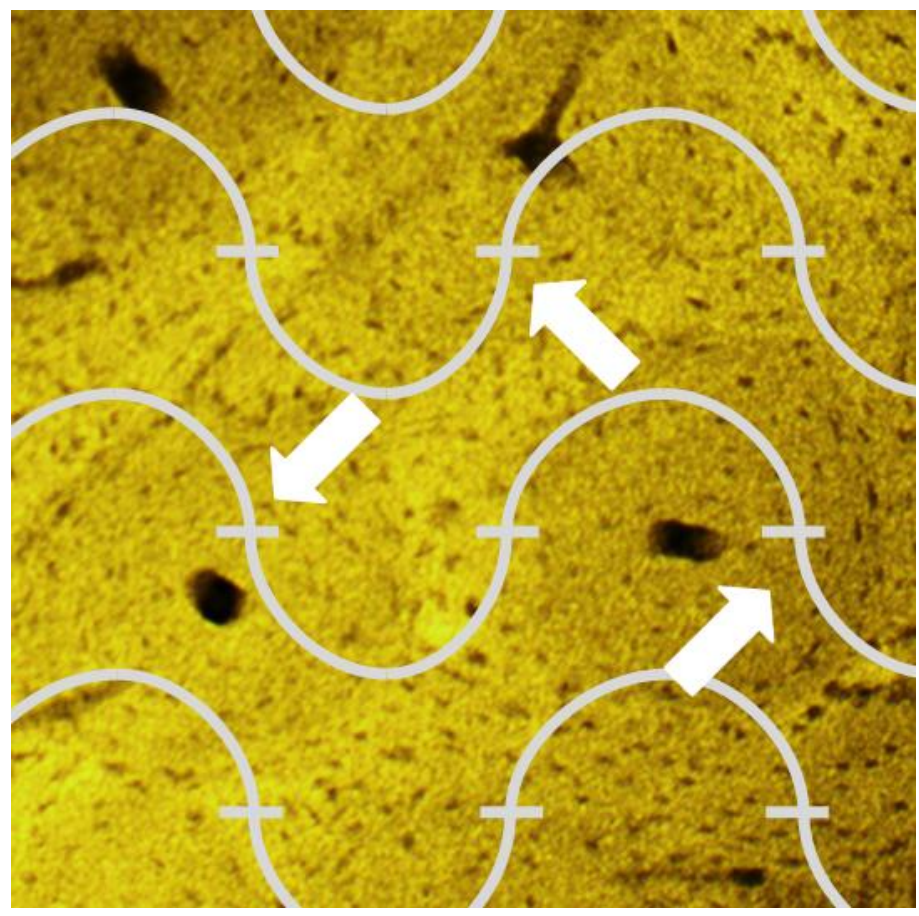

Figure 24. Merz grid points located on unencroached secondary bone.

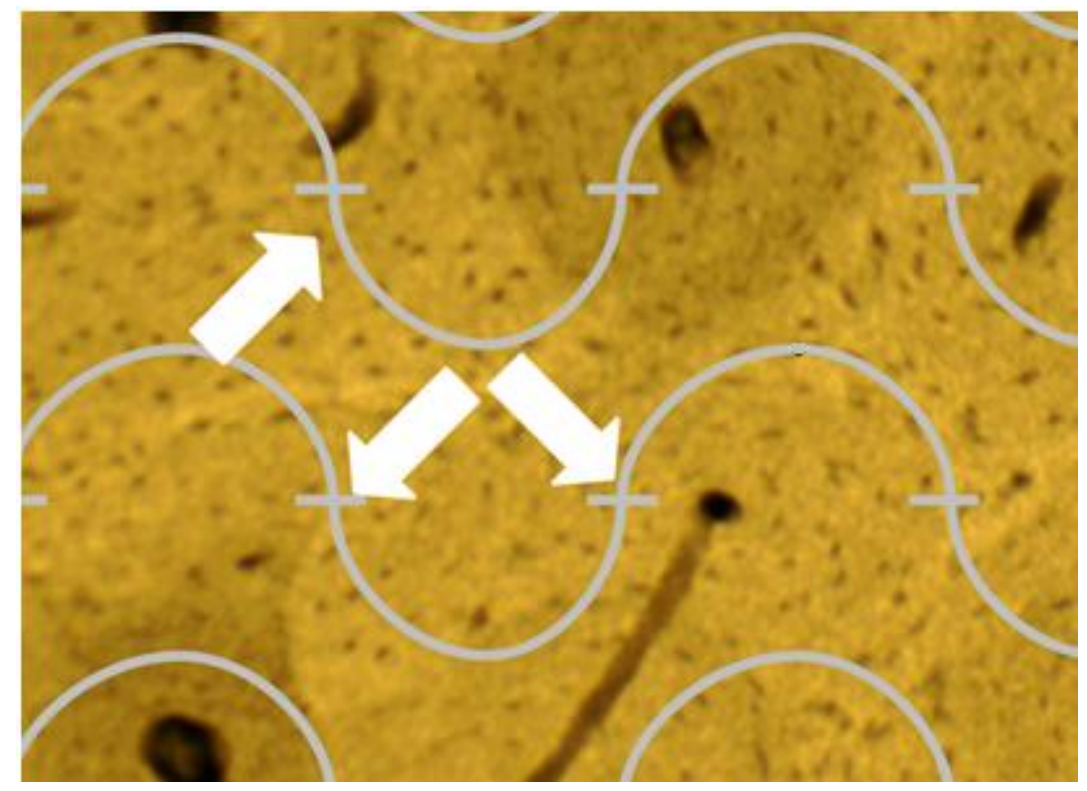

Figure 25. Merz grid points located on encroached secondary bone. 


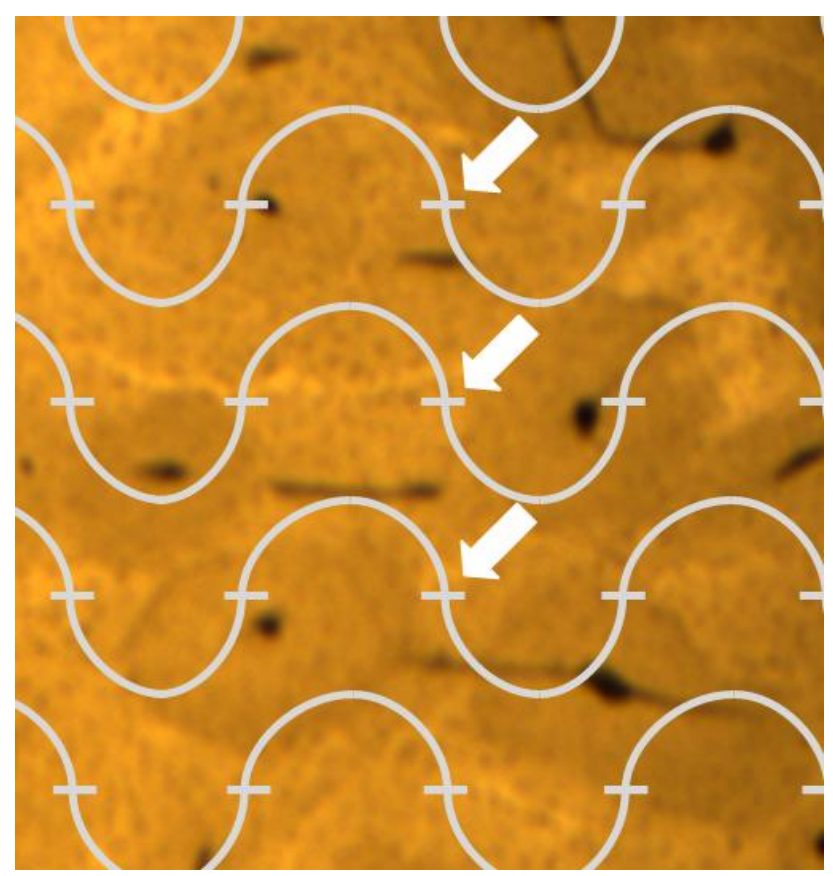

Figure 26. Another example of Merz grid points located on encroached secondary bone.

\subsubsection{Osteon Count Measurements}

In each of the four quadrants, the number of unencroached secondary osteons and the number of encroached secondary osteons were counted and recorded. The Merz grid was used as a guide and all of the unencroached and encroached secondary osteons that fell within the borders of the grid were counted (Figure 27). However to avoid counting secondary osteons multiple times, secondary osteons that fell on the borders were only counted if they fell on the top and right borders and not counted if they fell on the left or bottom borders of the grid. Unencroached secondary osteons were identified as intact secondary bone structures that have not been remodeled away at all by other secondary bone or resorption cavities, have a Haversian canal present, and have a clear unobstructed cement line. Encroached secondary osteons were identified as secondary bone structures which appear to have any section remodeled away by subsequent generation of osteons or resorption cavities, part of, all, or none of the Haversian 
canal can be remodeled away, and the cement line is not complete and can show concave sections.

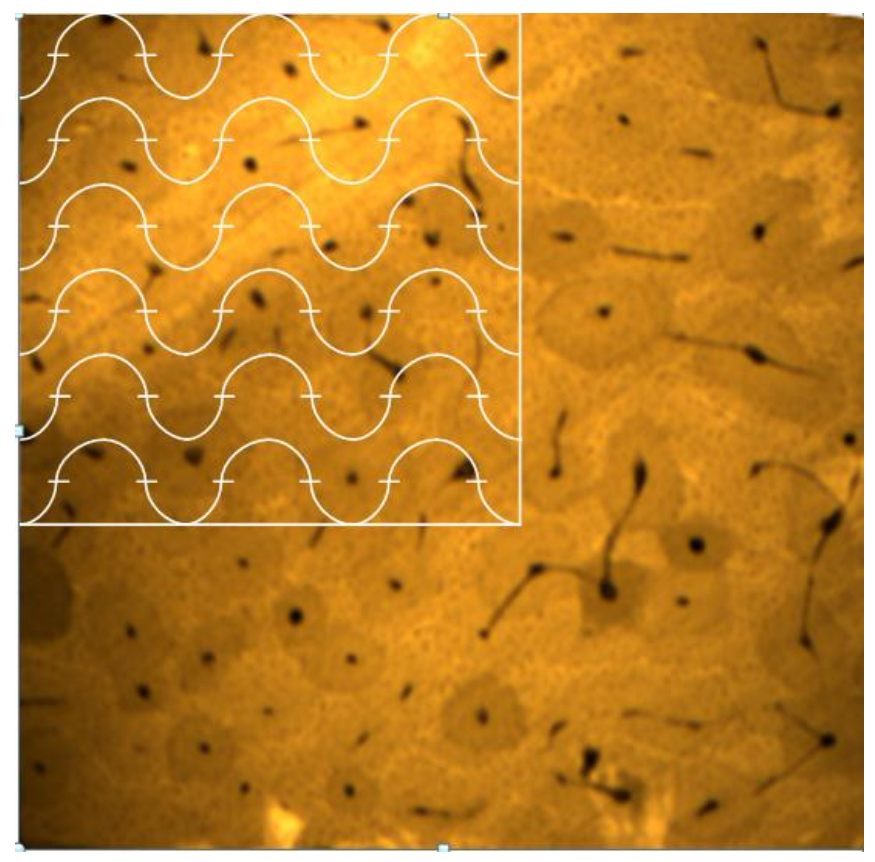

Figure 27. The field of view for the number of encroached secondary osteons and unencroached secondary osteons to be measured.

\subsubsection{Parameters}

The raw measurements collected from the methods previously described were then used to calculate 18 different parameters to better explain what was occurring in the tissue. The raw measurements for the 4 quadrants of each sector were averaged and those averaged values were used to calculate the parameters. These parameters included:

- $\mathrm{BV} / \mathrm{TV}$

- Fraction remodeled (as a tissue parameter)

- Fraction remodeled (as a material parameter)

- Fraction encroached (as a tissue parameter) 
- Fraction encroached (as a material parameter)

- Fraction encroached (as a remodeled parameter)

- Fraction unencroached (as a tissue parameter)

- Fraction unencroached (as a material parameter)

- Fraction unencroached (as a remodeled parameter)

- Secondary osteons per tissue area

- Secondary osteons per material area

- Encroached secondary osteons per tissue area

- Encroached secondary osteons per material area

- Unencroached secondary osteons per tissue area

- Unencroached secondary osteons per material area

- Average area of a secondary osteon

- Average area of an encroached secondary osteon

- Average area of an unencroached secondary osteon

BT/TV and the fraction remodeled, fraction encroached and fraction unencroached parameters are calculated with the point count measurements which explain what percentage of the remodeled area is involved with encroaching. Secondary osteons, encroached secondary osteons, and unencroached secondary osteons per area, as well as the average area of osteons, are calculated with the osteon count measurements which explain how the remodeling and encroachment is happening and the size of the osteons.

The porosity point count measurements are used to calculate the ratio of bone volume to tissue volume (BV/TV). BV/TV is calculated by dividing the number of porosity points by the number of points on the Merz grid and subtracting that number from one (Equation 1). 


$$
\frac{B V}{T V}=1-\frac{\text { porosity }}{36}(E q .1)
$$

The point count measurements for unencroached secondary osteons and encroached secondary osteons are used to calculate the fraction encroached and fraction unencroached in the tissue, material, and remodeled regions. The points of unencroached secondary osteons and encroached secondary osteons are also summed together to calculated the fraction remodeled in the tissue and material regions. The tissue region includes the entire section, the material region includes the entire section except for the porosity points, and the remodeled region includes the entire section except for the porosity and primary bone points (or the points that are encroached and unencroached secondary bone). The fraction remodeled, fraction encroached, and fraction unencroached for all regions can be seen below (Equations 2-9).

$$
\begin{gathered}
\text { Fraction Remodeled }(\text { tissue })=\frac{\text { unencroached }+ \text { encroached }}{36}(\text { Eq. } 2) \\
\text { Fraction Remodeled }(\text { material })=\frac{\text { unencroached }+ \text { encroached }}{36-\text { porosity }}(\text { Eq. } 3) \\
\text { Fraction Encroached }(\text { tissue })=\frac{\text { encroached }}{36}(\text { Eq. } 4) \\
\text { Fraction Encroached }(\text { material })=\frac{\text { encroached }}{36-\text { porosity }}(\text { Eq. } 5) \\
\text { Fraction Encroached }(\text { remodeled })=\frac{\text { encroached }}{\text { encroached }+ \text { unencroached }}(\text { Eq. } 6) \\
\text { Fraction Unencroached }(\text { tissue })=\frac{\text { unencroached }}{36}(\text { Eq. } 7) \\
\text { Fraction Unencroached }(\text { remodeled })=\frac{\text { unencroached }}{\text { encroached }+ \text { unencroached }}(\text { Eq. } 9)
\end{gathered}
$$


The encroached secondary osteon count measurements are used to calculate the encroached secondary osteons per area, the unencroached secondary osteon count measurements are used to calculate the unencroached secondary osteons per area, and the sum of the encroached and unencroached secondary osteons are used to calculate the secondary osteons per area. In order to calculate these parameters, the field of view area of the Merz grid at the 10X objective was calculated. This was done by looking through the Merz grid, focusing the specimen at the 10X objective and then measuring the sides of the Merz grid with the stage micrometer. The area of the field of view is the tissue area and the area multiplied by BV/TV is the material area. The equations for secondary osteons, encroached secondary osteons, and unencroached secondary osteons per both areas can be seen below (Equations 10-18)

$$
\begin{gathered}
\frac{\text { Secondary Osteons }}{\text { tissue area }}=\frac{\text { unencroached osteons }+ \text { encroached osteons }}{\text { field area }}(\text { Eq. 10) } \\
\frac{\text { Secondary Osteons }}{\text { material area }}=\frac{\text { unencroached osteons }+ \text { encroached osteons }}{\text { field area } * B V / T V}(E q .11) \\
\frac{\text { Encroached Secondary Osteons }}{\text { tissue area }}=\frac{\text { encroached osteons }}{\text { field area }}(E q .12) \\
\frac{\text { Encroached Secondary Osteons }}{\text { material area }}=\frac{\text { encroached osteons }}{\text { field area } * \text { BV } / T V}(E q .13) \\
\frac{\text { Unencroached Secondary Osteons }}{\text { material area }}=\frac{\text { unencroached osteons }}{\text { field area } * \text { BV } / T V}(E q .15)
\end{gathered}
$$

To determine the average area per osteon the remodeled area needs to be calculated, which is the area of the field of view multiplied by the fraction remodeled as a tissue parameter. This area is then divided by the osteon count measurements of the number of encroached secondary osteons, unencroached secondary osteons, or the sum of them both to determine the 
average area per osteon (Equations 16-18). The inverse of the parameters for the average area per secondary osteon, encroached secondary osteon, and unencroached secondary osteon can be calculated to determine the number of secondary osteons, encroached secondary osteons, and unencroached secondary osteons per remodeled area.

$$
\begin{gathered}
\text { Secondary Osteon Avg. Area }=\frac{\text { field area } * \text { Fraction Remodeled }(\text { tissue })}{\text { unencroached osteons }+ \text { encroached osteons }}(E q .16) \\
\text { Encroached Secondary Osteon Avg. Area }=\frac{\text { field area } * \text { Fraction Remodeled }(\text { tissue })}{\text { encroached osteons }}(E q .17) \\
\text { Unencroached Secondary Osteon Avg. Area }=\frac{\text { field area } * \text { Fraction Remodeled }(t i s s u e)}{\text { unencroached osteons }}(E q .18)
\end{gathered}
$$

\subsection{Statistical Analysis}

To analyze each of the calculated parameters from the point count and osteon count measurements, a 2-way Repeated-Measures ANOVA in SigmaPlot 12.3 (Systat Software, Inc.) was used. The ANOVA model evaluated the effect of the anatomical sector (tensile and compressive) and the type of surgery the ewe underwent (OVX and sham) for each of the parameters. The interaction between sector and surgery was evaluated as well. These comparisons were calculated with a post-hoc Fisher Least Significant Difference (LSD) test. Pvalues less than 0.05 were considered significant for the main effects and for the post-hoc Fisher LSD tests and p-values less than 0.10 were considered significant for the interaction. 


\section{RESULTS}

\subsection{Repeatability}

Measurements were made 3 separate times on the compressive side to make sure that the measurements were repeatable. A one way repeated measures ANOVA was run to compare each of the measurements to determine if there was a significant difference between groups. The pvalues from these tests can be seen in table 2 . The column labeled, 1 vs. 2 , is the p-value from the LSD Fisher post hoc test comparing measurements 1 to measurements 2.1 vs. 3 is comparing measurements 1 to measurements 3.2 vs. 3 is comparing measurements 2 to measurements 3.The first measurements were sometimes significantly different than the last two rounds of measurements, but the second and third measurements were not significantly different from each other. The similarity in measurements in the second and third round showed that these methods developed were repeatable. For analysis of these data, the third measurements were used.

Table 2. P-values found from one way repeated measures ANOVAs determining if there is a significant difference between measurements.

\begin{tabular}{|l|c|c|c|c|}
\hline \multicolumn{1}{|c|}{ Measurement } & ANOVA & 1 vs. 2 & $\mathbf{1}$ vs. 3 & $\mathbf{2}$ vs. 3 \\
\hline \multicolumn{4}{|c|}{ POINT COUNT METHOD } \\
\hline Porosity & 0.365 & & & \\
\hline Primary Bone & 0.001 & 0.003 & $<0.001$ & 0.508 \\
\hline Unencroached Bone & 0.062 & & & \\
\hline Encroached Bone & $<0.001$ & $<0.001$ & $<0.001$ & 0.583 \\
\hline \multicolumn{4}{|c|}{ OSTEON COUNT METHOD } \\
\hline Unencroached Osteons & 0.931 & & & \\
\hline Encroached Osteons & 0.004 & 0.003 & 0.005 & 0.780 \\
\hline
\end{tabular}

\subsection{Two Way Repeated Measures ANOVA}

Two way repeated measures ANOVAs were run for each of the parameters calculated from the raw data to determine if the sector or surgery had a significant effect. Table 3 displays 
the p-values from those ANOVAs, as well as the p-values calculated from the LSD Fisher post hoc tests for the effects of sector, surgery, and the interaction between them both. The results showed that sector, surgery, and the interaction had significant effects for a variety of different parameters. Further discussion and a graph display for each of the parameters will be given in the next section.

Table 3. P-values found from two way repeated measures ANOVAs for each parameter determining if sector, surgery, or the interaction have a significant effect.

\begin{tabular}{|l|c|c|c|}
\hline \multicolumn{4}{|c|}{ 2-way Repeated Measures ANOVA: p-values } \\
\hline \multicolumn{1}{|c|}{ Parameter } & Sector & Surgery & Interaction \\
\hline BV/TV & 0.37 & 0.568 & 0.62 \\
\hline Fraction Remodeled (Tissue) & 0.308 & 0.017 & 0.024 \\
\hline Fraction Remodeled (Material) & 0.192 & 0.011 & 0.01 \\
\hline Fraction Encroached (Tissue) & 0.04 & 0.19 & 0.026 \\
\hline Fraction Encroached (Material) & 0.026 & 0.177 & 0.017 \\
\hline Fraction Encroached (Remodeled) & 0.032 & 0.904 & 0.214 \\
\hline Fraction Unencroached (Tissue) & 0.638 & 0.025 & 0.159 \\
\hline Fraction Unencroached (Material) & 0.725 & 0.015 & 0.109 \\
\hline Fraction Unencroached (Remodeled) & 0.032 & 0.904 & 0.214 \\
\hline Secondary Osteons/ Tissue Area & 0.683 & 0.264 & 0.03 \\
\hline Secondary Osteons/ Material Area & 0.822 & 0.225 & 0.018 \\
\hline Encroached Secondary Osteons/ Tissue Area & 0.43 & 0.152 & 0.022 \\
\hline Encroached Secondary Osteons/Material Area & 0.326 & 0.145 & 0.015 \\
\hline Unencroached Secondary Osteons/ Tissue Area & 0.094 & 0.856 & 0.096 \\
\hline Unencroached Secondary Osteons/ Material Area & 0.118 & 0.781 & 0.062 \\
\hline Secondary Osteon Average Area & 0.079 & 0.006 & 0.943 \\
\hline Encroached Secondary Osteon Average Area & 0.882 & 0.895 & 0.384 \\
\hline Unencroached Secondary Osteon Average Area & 0.010 & 0.002 & 0.511 \\
\hline
\end{tabular}

\subsection{Point Count Measurements}

The ANOVA found that there was no change in BV/TV based on whether the sector of the bone was from a region where it was normally in compression or in tension, or if the ewe had 
an ovariectomy surgery or a sham surgery. These results can be seen in Figure 28. This indicates that there is no demonstrable change in architecture from ovariectomy or regional changes.

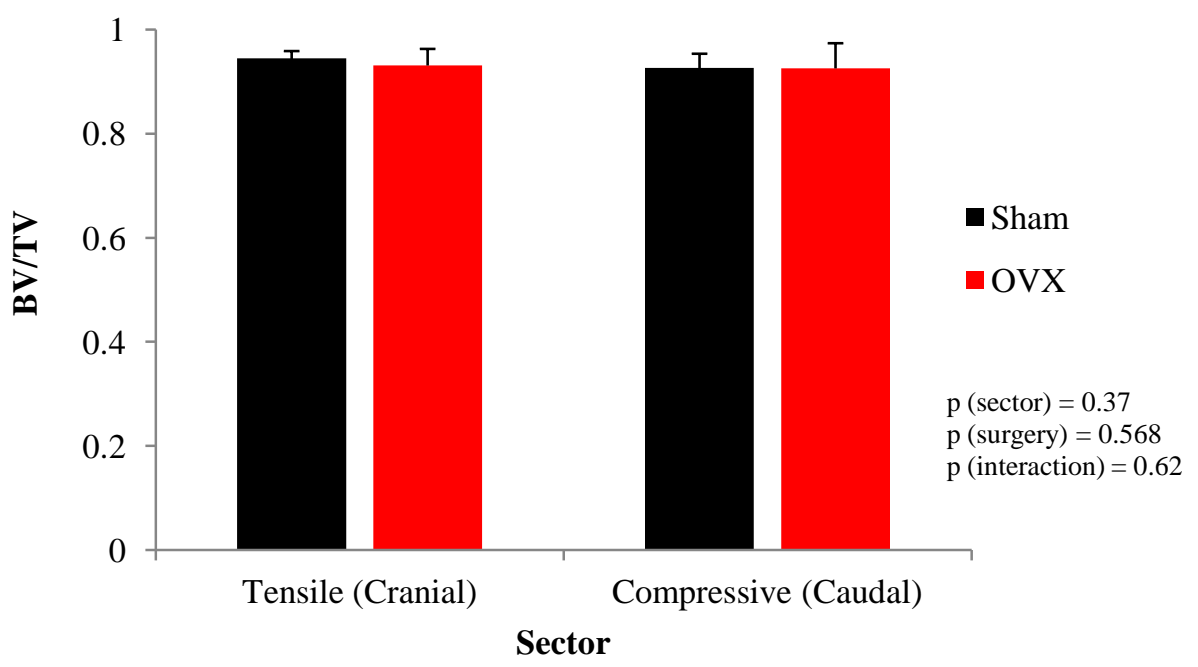

Figure 28. The mean BV/TV with the standard deviation for the sham and OVX sheep for each sector.

The fraction remodeled, as both a tissue parameter and a material parameter, showed to be significantly affected from the type of surgery the ewe underwent and the interaction term (Figure 29 and Figure 30). For the sham group only, bone sectors in regions with compressive loading was found to have a higher fraction remodeled than bone sectors in regions with tensile forces. However, when the ewe underwent an ovariectomy, there was no difference in the fraction remodeled for the different regions. Also, the ovariectomy surgery was associated with a larger fraction remodeled compared to the sham on the tensile side, but the type of surgery had no demonstrable effect on the compressive side. This could indicate that on the tensile side, the remodeling in the OVX animals is now happening in primary bone, or places where remodeling did not occur in the sham animals, as well as in places where remodeling has previously occurred, because this parameter describes what is occurring in the overall area of the section. 
On the compressive side, remodeling is occurring in places where remodeling has previously occurred for the OVX animals, so there may be no difference in the fraction remodeled between the two types of surgeries.

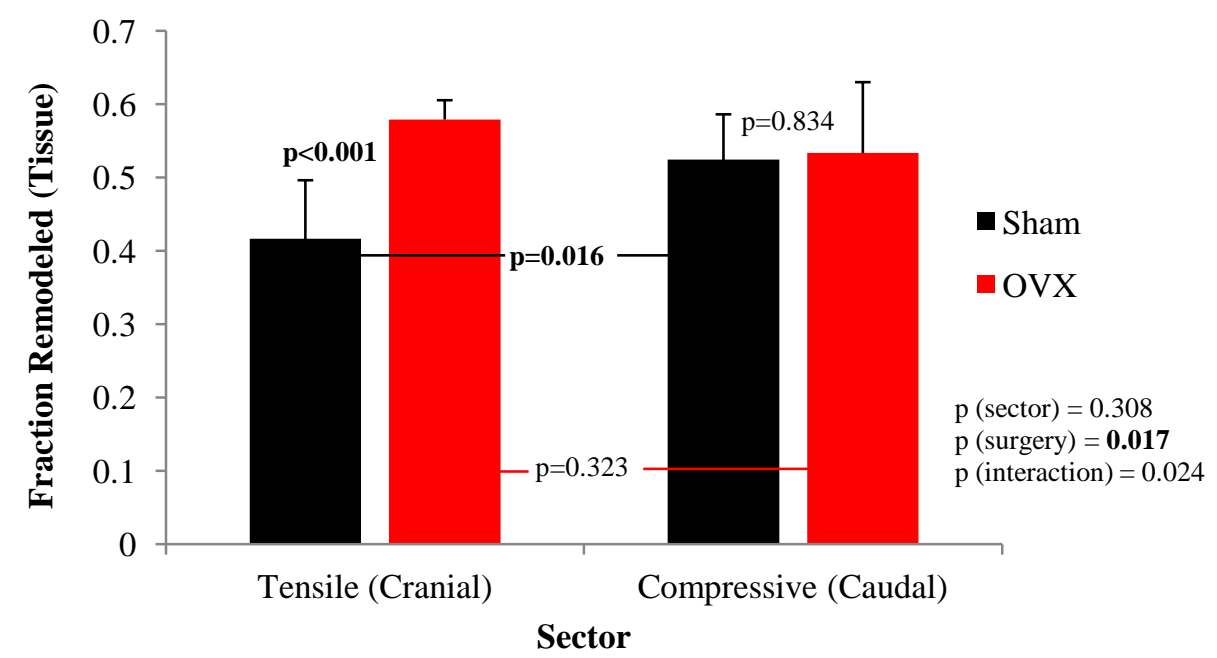

Figure 29. The mean fraction remodeled as a tissue parameter with the standard deviation for the sham and OVX sheep for each sector. 


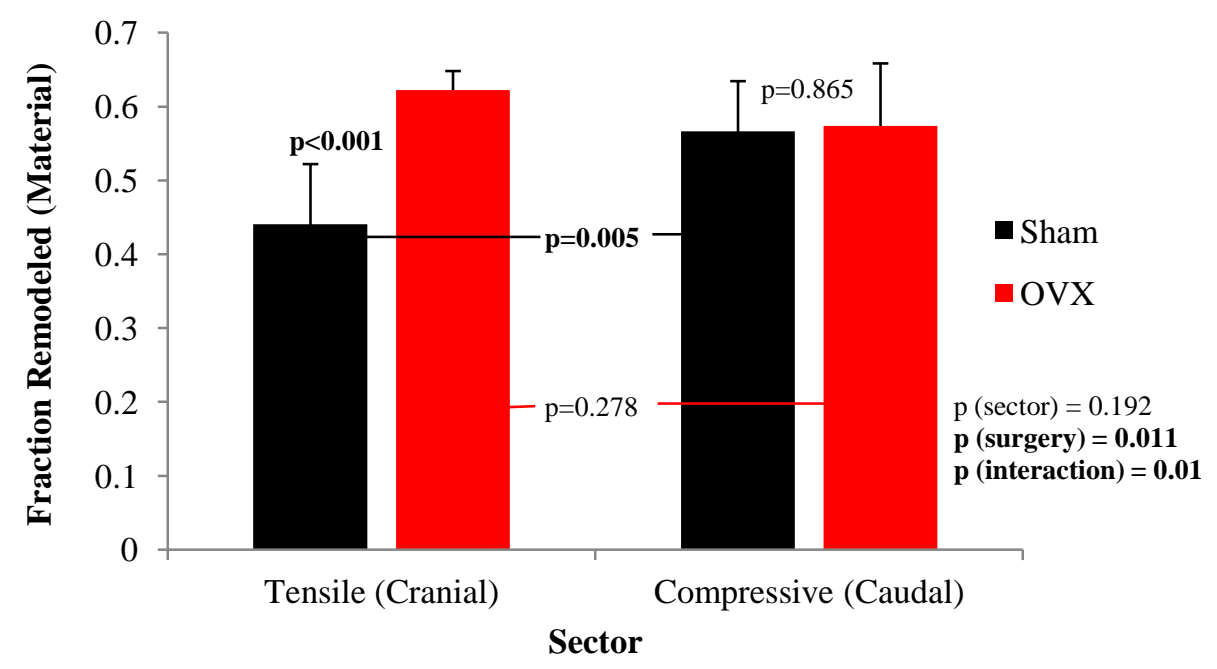

Figure 30. The mean fraction remodeled as a material parameter with the standard deviation for the sham and OVX sheep for each sector.

The fraction encroached as both a tissue parameter and a material parameter showed to be significantly affected by the sector and the interaction term (Figure 31 and Figure 32). For the sham group only, there was a significantly larger fraction encroached on the compressive side compared to the tensile side. On the other hand, when the animal had an ovariectomy, there was no difference in the fraction encroached when comparing sides. Also, on the tensile side only, there was a significant increase in the fraction encroached when the animal had an ovariectomy compared to a sham surgery. This could indicate that on the tensile side, more remodeling is occurring as a result of the ovariectomy surgery in places that have had remodeling before and also at the borders of the remodeled area and primary bone, which would increase the fraction encroached. For the compressive side, remodeling is occurring in places that have had remodeling before, so no change in the fraction encroached was found. The significant differences found for the fraction encroached as a tissue and material parameter were the same significant differences found for the fraction remodeled as a tissue and as a material parameter. 


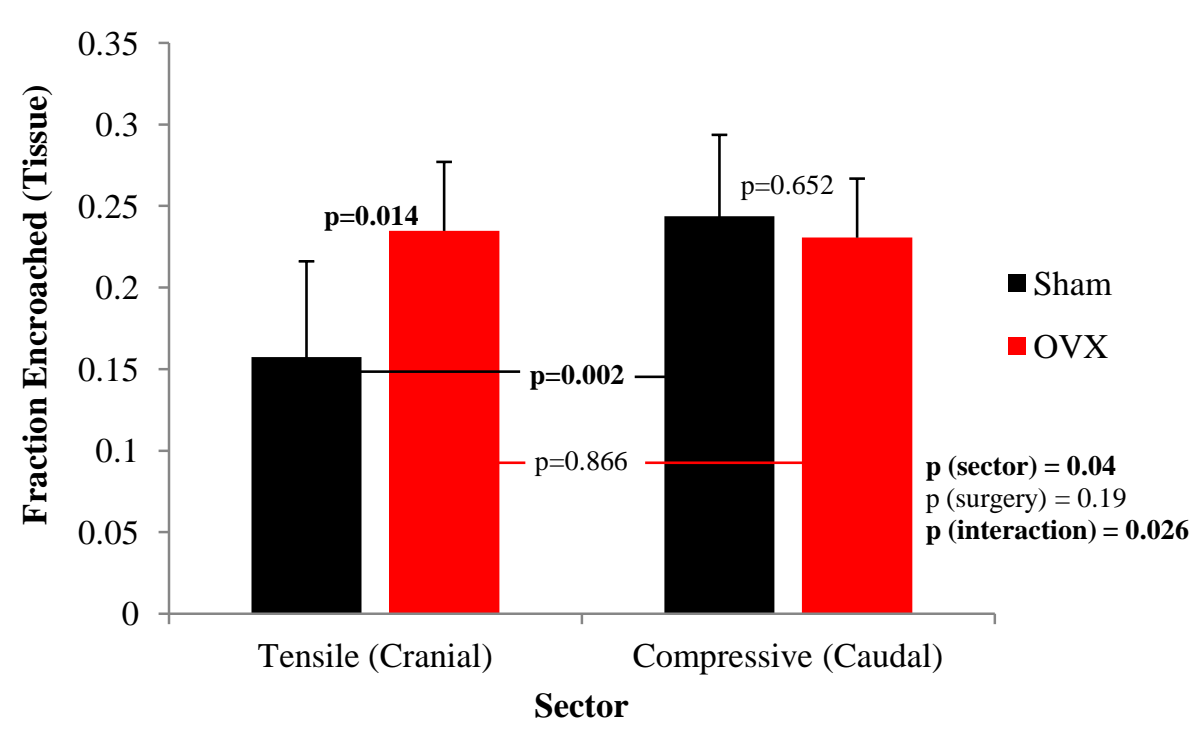

Figure 31. The mean fraction encroached as a tissue parameter with the standard deviation for the sham and OVX sheep for each sector.

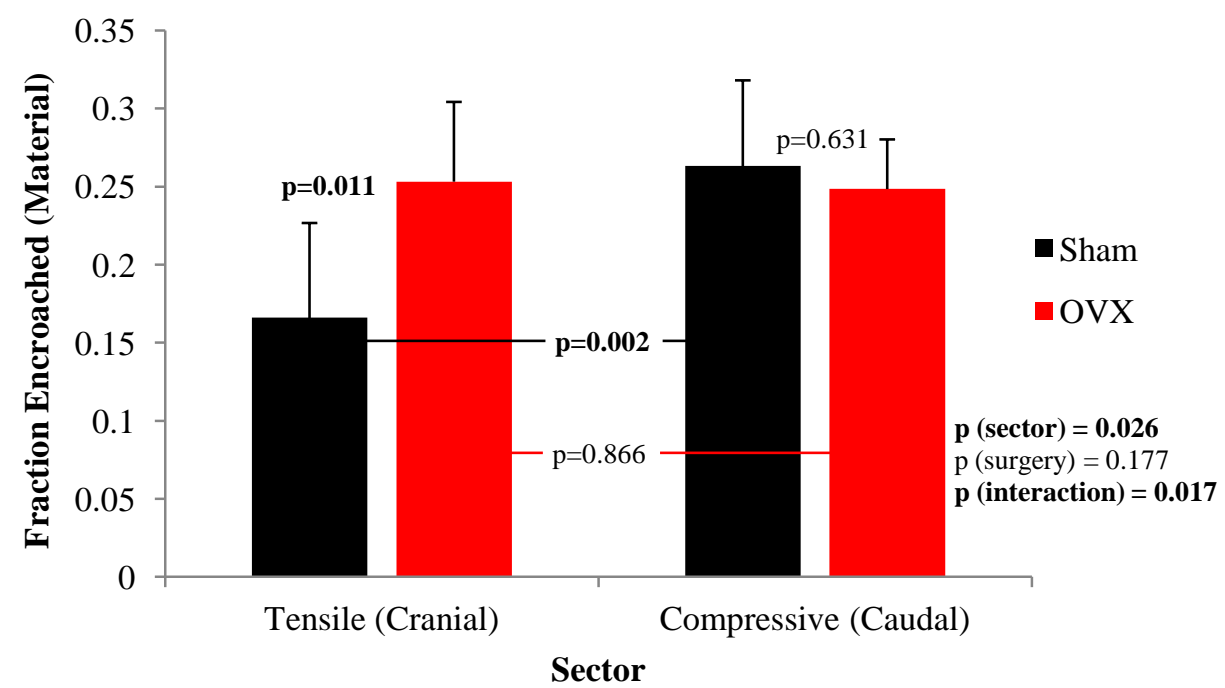

Figure 32. The mean fraction encroached as a material parameter with the standard deviation for the sham and OVX sheep for each sector.

When just looking at the remodeled area, the fraction encroached showed to be significantly affected by the sector, with the compressive side showing a larger fraction 
encroached (Figure 33). This could indicate that more remodeling has occurred on the compressive side compared to the tensile side, especially in areas where there was prior remodeling, because a larger proportion of the remodeled area has been removed by newer secondary bone structures. The results from this parameter also show that about half of the remodeled area on the compressive side, for both OVX and sham, is encroached secondary bone.

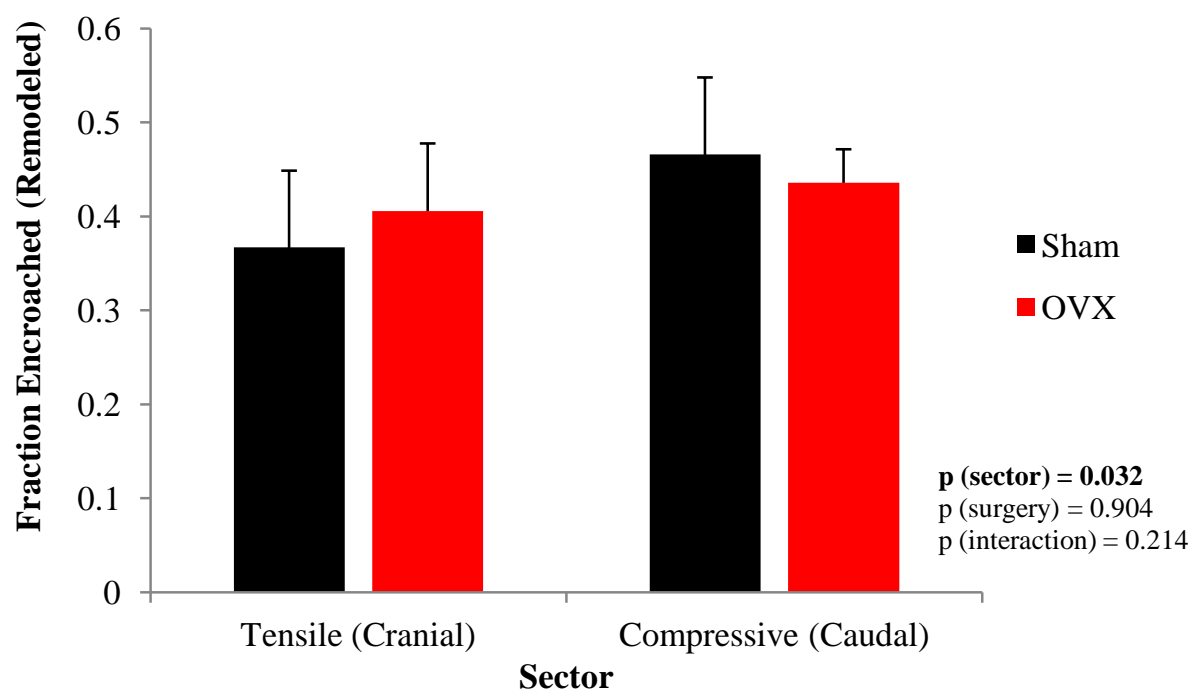

Figure 33. The mean fraction encroached as a remodeled parameter with the standard deviation for the sham and OVX sheep for each sector.

The fraction unencroached as both a tissue parameter and a material parameter showed to be significantly affected by the type of surgery, with the ovariectomy showing a larger fraction unencroached (Figure 34 and Figure 35). This can indicate that for both sectors, the ovariectomy surgery results in an increase in the area of the tissue or material that has new secondary osteons. 


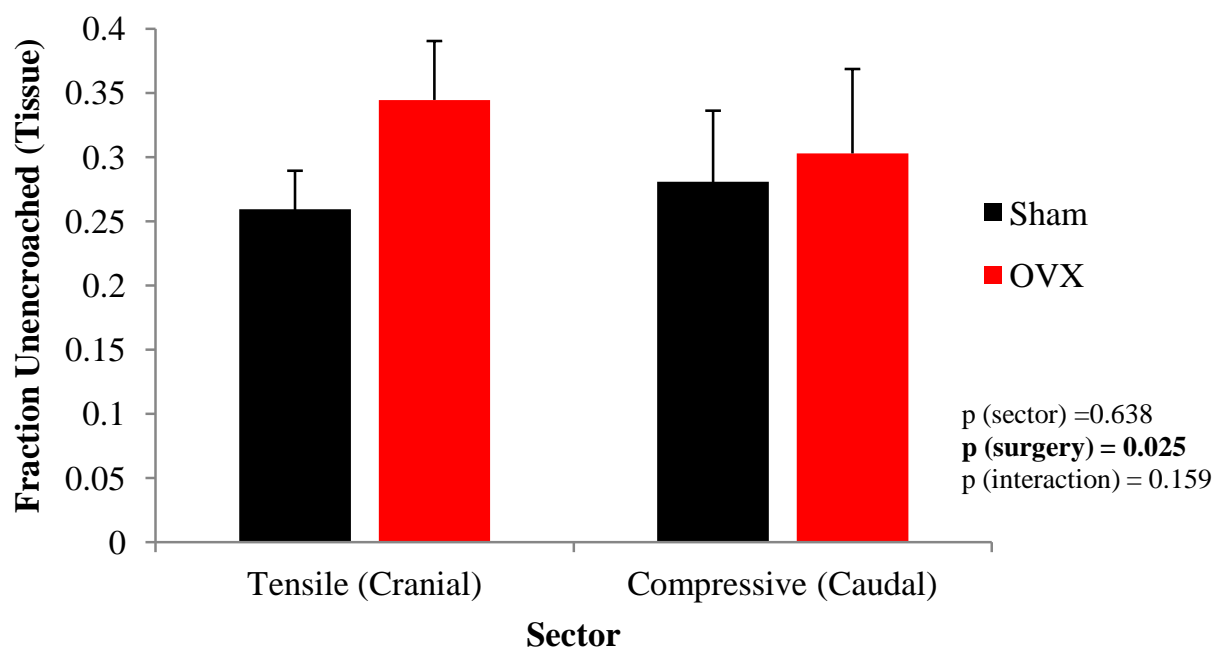

Figure 34. The mean fraction unencroached as a tissue parameter with the standard deviation for the sham and OVX sheep for each sector.

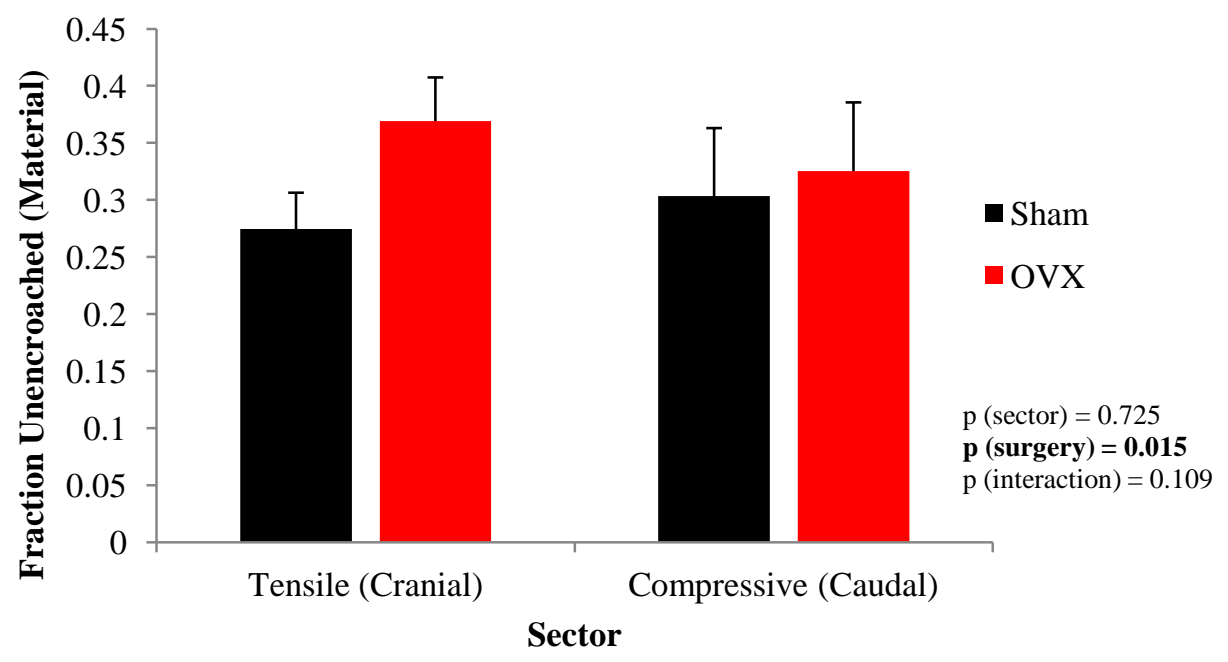

Figure 35. The mean fraction unencroached as a material parameter with the standard deviation for the sham and OVX sheep for each sector.

The fraction unencroached, as a remodeled parameter, showed to be significantly affected by the sector with the tensile side showing a larger fraction unencroached (Figure 36). When 
analyzed with Figure 33, the results show that the compressive side had more remodeling previously as seen from the larger fraction encroached. On the tensile side, a larger proportion of the remodeled area is new, as seen from the larger fraction unencroached. The fraction unencroached and the fraction encroached, as remodeled parameters, sum up to 1, and therefore they tell us the same information.

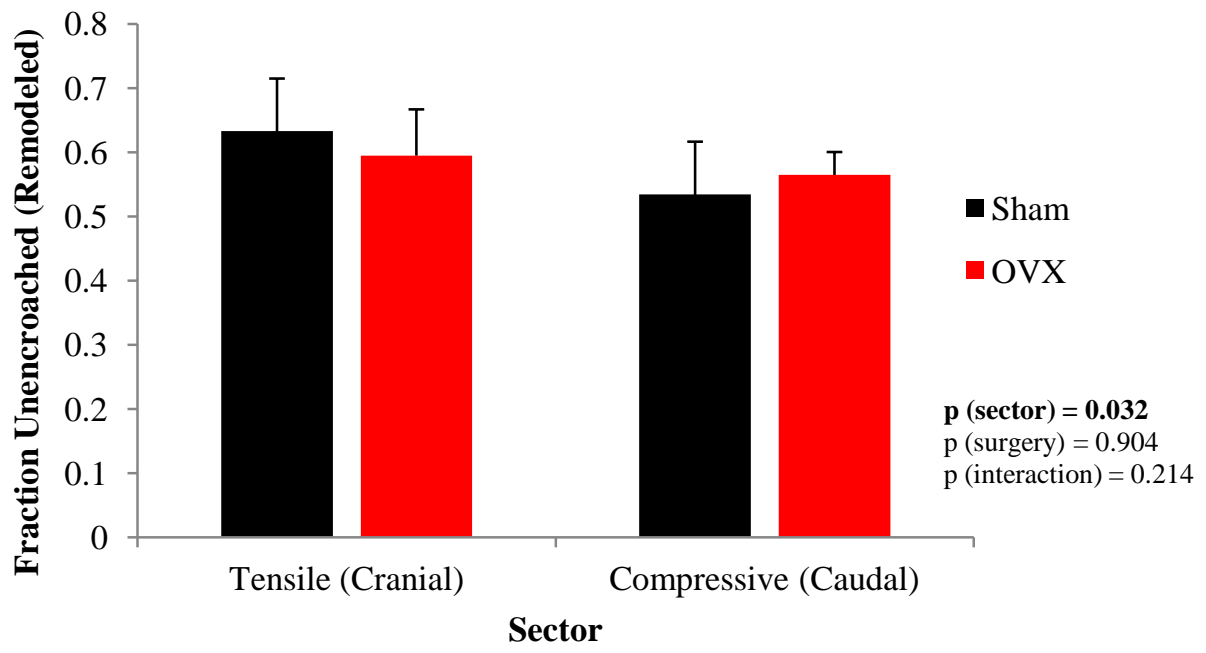

Figure 36. The mean fraction unencroached as a remodeled parameter with the standard deviation for the sham and OVX sheep for each sector.

\subsection{Osteon Count Measurements}

The number of secondary osteons per both tissue area and material area showed to be significantly affected by the interaction term (Figure 37 and Figure 38). The number of secondary osteon per area, on the tensile side only, was larger for the OVX animals compared to the sham animals. This could indicate that more remodeling is occurring from an ovariectomy surgery on the tensile side or that more remodeling is occurring in places where it didn't occur before. 


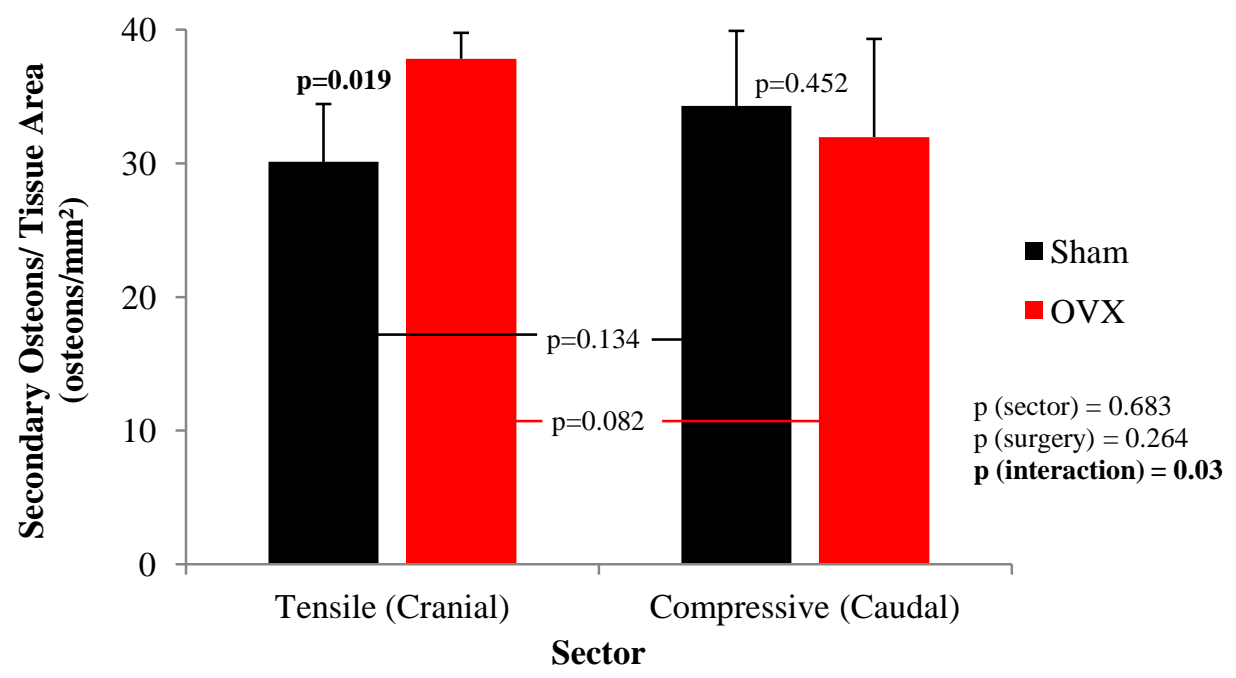

Figure 37. The mean number of secondary osteons per tissue area with the standard deviation for the sham and OVX sheep for each sector.

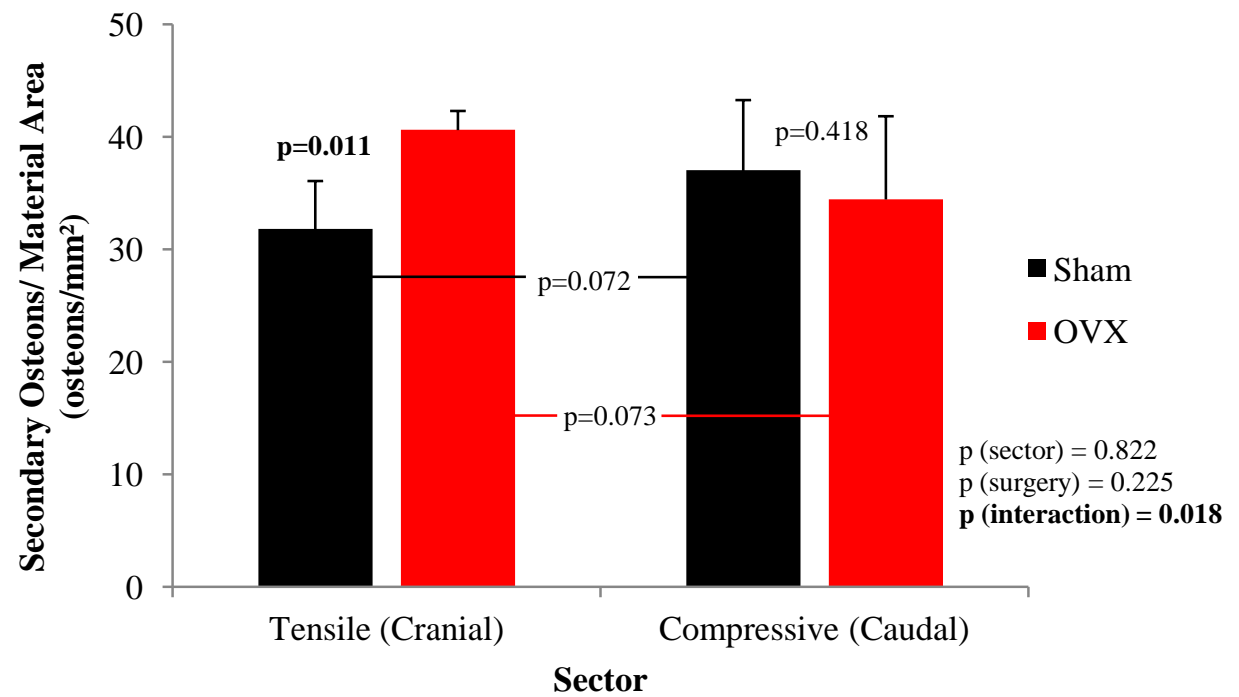

Figure 38. The mean number of secondary osteons per material area with the standard deviation for the sham and OVX sheep for each sector.

The number of encroached secondary osteons per both tissue area and material area showed to be significantly affected by the interaction term (Figure 39 and Figure 40). On the tensile side, the number of encroached osteons per both tissue area and material area was larger 
for the ovariectomized animals compared to the sham animals. Also, for the sham animals only, the compressive side had a larger number of encroached secondary osteons per area. These results are consistent with the results for the fraction encroached as a tissue and as a material parameter. This can support the same idea as above that on the tensile side, more remodeling is occurring as a result of the ovariectomy surgery in places that have had remodeling before, at the borders of the remodeled area and primary bone, and in primary bone, which would increase the number of encroached secondary osteons per area. For the compressive side, remodeling is occurring in places that have had remodeling before, so no change in the number of encroached secondary osteons per area was found.

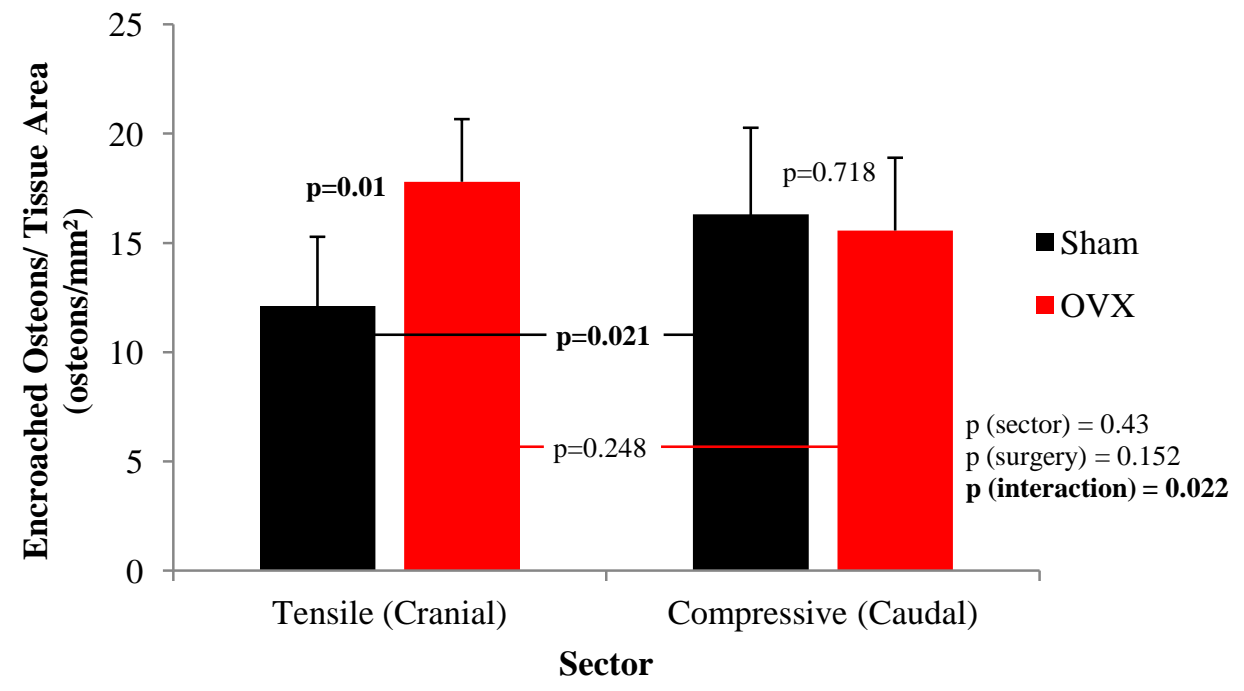

Figure 39. The mean number of encroached secondary osteons per tissue area with the standard deviation for the sham and OVX sheep for each sector. 


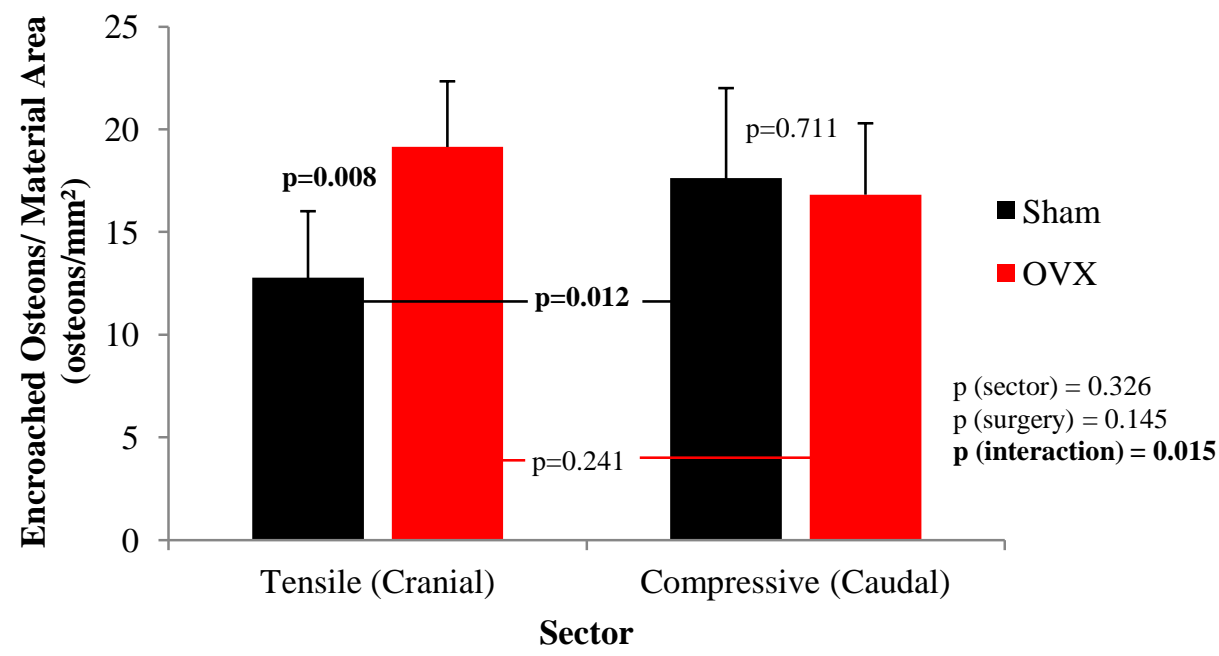

Figure 40. The mean number of encroached secondary osteons per material area with the standard deviation for the sham and OVX sheep for each sector.

The number of unencroached secondary osteon per both tissue area and material area showed to be significantly affected by the interaction term (Figure 41 and Figure 42). For the ovariectomized animals, the number of unencroached secondary osteons per tissue area and material area was larger on the tensile side than on the compressive side. This shows that there are more new osteons in the ovariectomized animals on the tensile side. These results, along with the results from the fraction unencroached support the idea that the new osteons are larger on the compressive side. This also may support the idea that from the ovariectomy surgery, the tensile side, which was bone that did not undergo that much remodeling before, is now acting like the compressive side and undergoing more remodeling, and possibly now less responsive to load. 


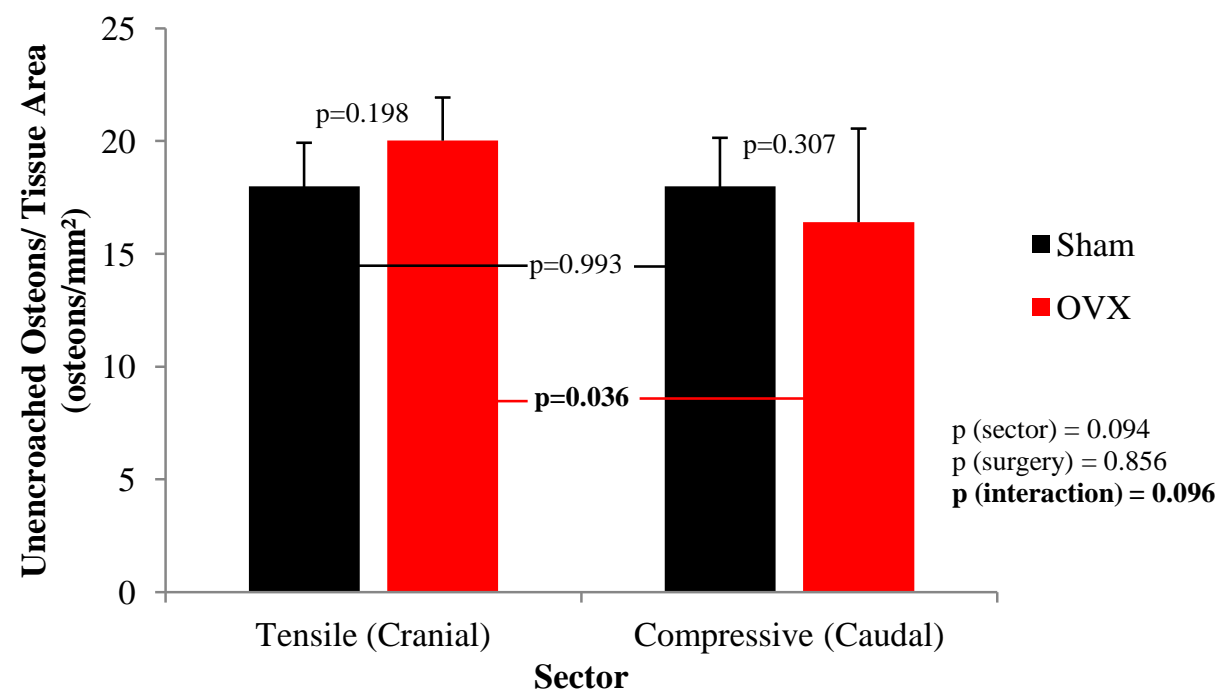

Figure 41. The mean number of unencroached secondary osteons per tissue area with the standard deviation for the sham and OVX sheep for each sector.

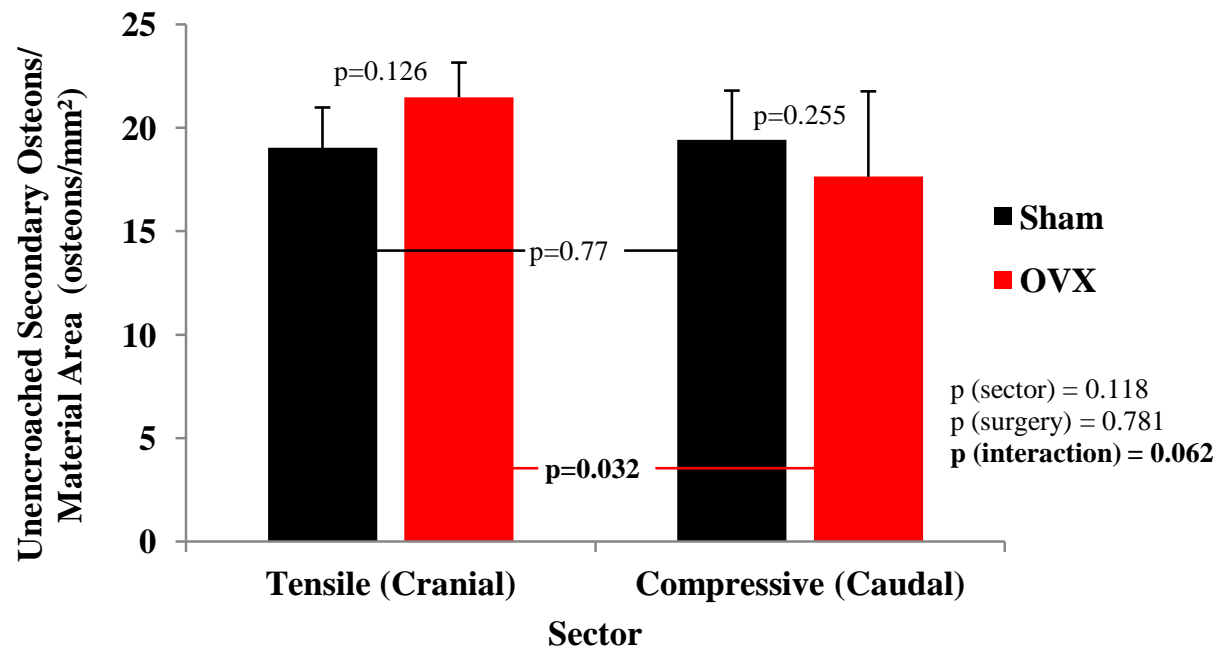

Figure 42. The mean number of unencroached secondary osteons per material area with the standard deviation for the sham and OVX sheep for each sector. 


\subsection{Osteonal Area}

The secondary osteon average area showed to be significantly affected by the type of surgery, with the ovariectomized animals having larger secondary osteons (Figure 43). This indicates that ovariectomy surgeries cause secondary osteons to be larger than if they did not have the surgery.

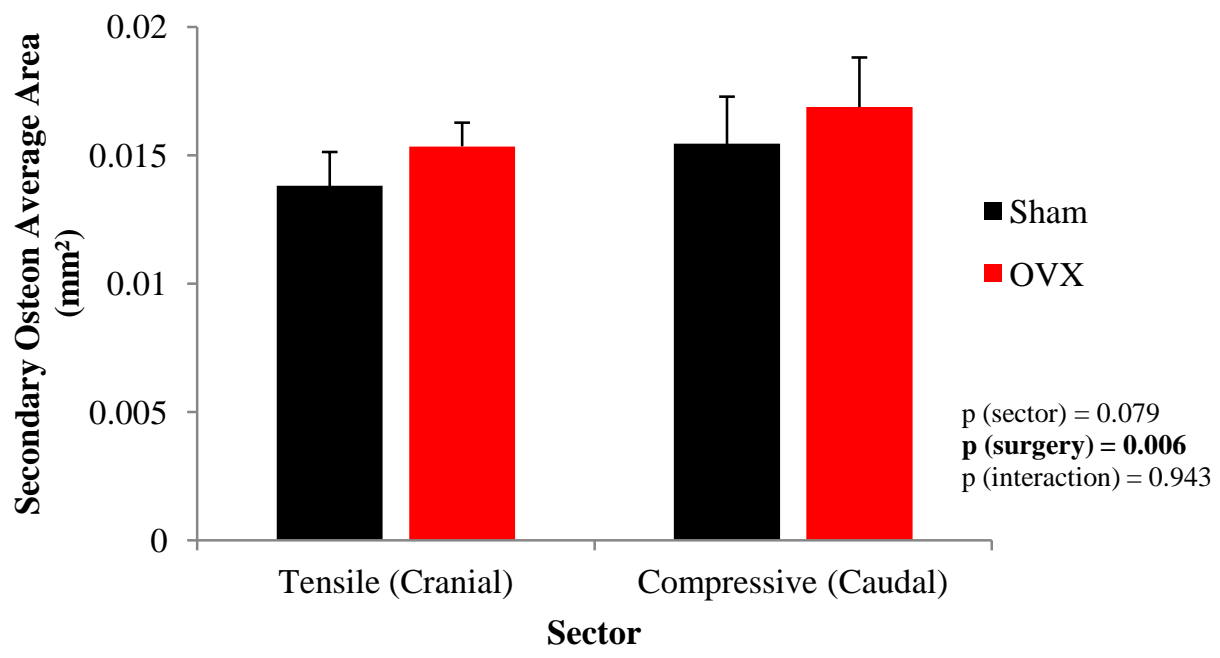

Figure 43. The mean secondary osteon average area with the standard deviation for the sham and OVX sheep for each sector.

The encroached secondary osteon average area showed no significant differences between surgery type or sector location (Figure 44). This should be the case, since the encroached osteons are the old osteons which have been remodeled away. The amount of area that gets removed away on each osteon does not depend on the surgery or the region. 


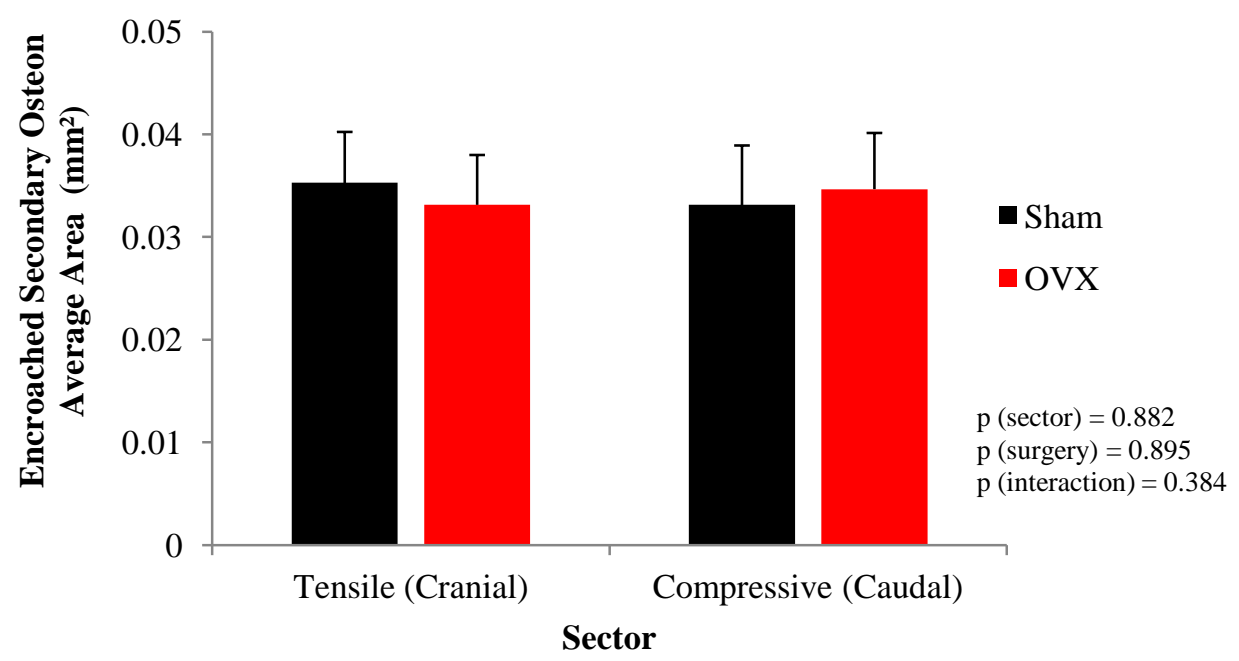

Figure 44. The mean encroached secondary osteon average area with the standard deviation for the sham and OVX sheep for each sector.

The unencroached secondary osteon average area showed to be significantly affected by both sector and surgery (Figure 45). The average area of unencroached osteons was larger on the compressive side than the tensile side, and the average area of the unencroached osteons was also larger for the ovariectomized animals than the sham animals. This indicates that ovariectomy surgery causes the newly formed osteons to be larger than they would have been without the surgery. It also indicates that osteons tend to be larger in regions of compression than in regions of tension. 


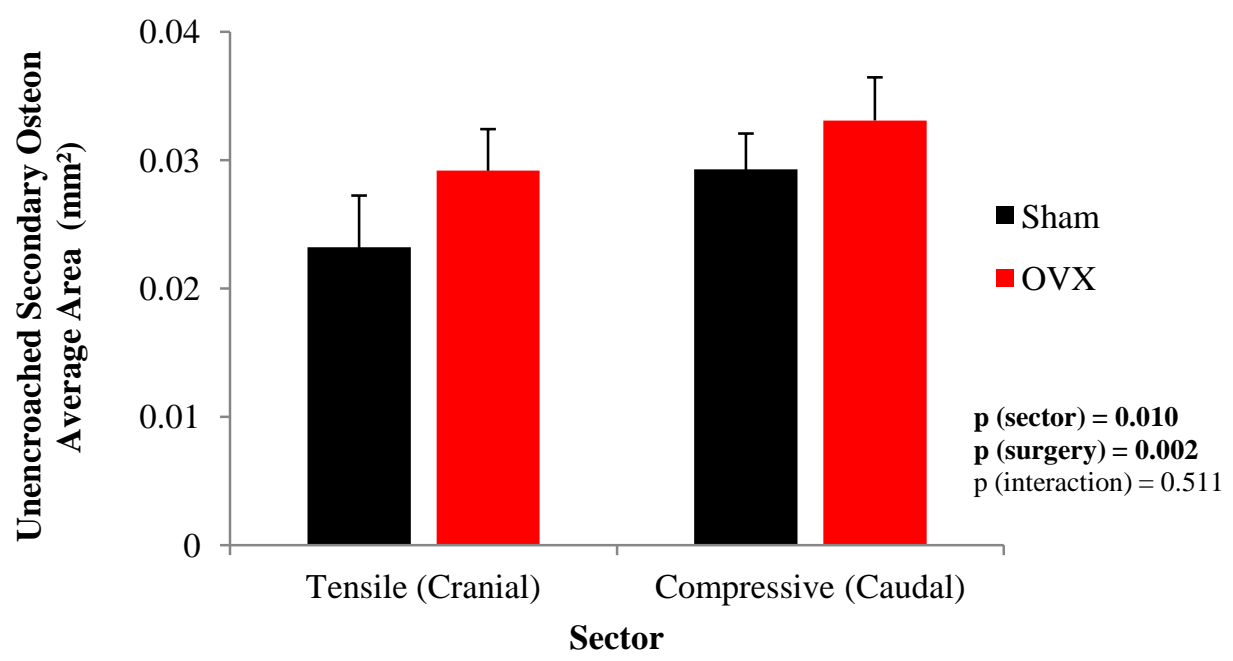

Figure 45. The mean unencroached secondary osteon average area with the standard deviation for the sham and OVX sheep for each sector. 


\section{DISCUSSION}

\subsection{Postmenopausal Osteoporosis Ovine Model}

Osteoporosis is a major health concern, especially in postmenopausal women. Animal models to test new solutions to help stop or slow down this disease are needed. Sheep models are a good choice since their bone undergoes Haversian remodeling and they have a similar hormone profile to humans. In this study, removal of the sheep's ovaries was preformed to simulate postmenopause, and sheep were sacrificed after 12 months to undergo bone analysis. These results did show an increase in remodeling in certain regions as a result of the removal of ovaries, however there was not a change in the porosity.

Osteoporosis is characterized by an increase in porosity. When looking at this osteoporosis definition alone, the sheep model is not an ideal model for testing human postmenopausal osteoporosis treatment options. Nevertheless, the sheep model does show a change in bone remodeling resulting in an animal model of postmenopausal osteoporosis that does not lose any bone for a time period of one year. This model could be further studied to determine what the sheep are doing that prevents the bone from experiencing a decrease in porosity. This knowledge could be greatly beneficial for postmenopausal osteoporosis and has the potential to be utilized for human treatment plans to prevent bone loss.

\subsection{Hypothesis Evaluation and Results Interpretation}

One of the goals of this study was to develop a repeatable method to quantify osteonal encroachment. Based on the results of the repeatability study, the second and third round measurements showed that these methods developed were repeatable, however, the first round measurements showed to be significantly different for some of the measurements. This was most 
likely due to the fact that it was the first time the methods were used, so the consistency of the measurements was still being developed.

This study shows interesting and new findings due to the evaluation of secondary bone as either encroached or unencroached, however, the initial hypothesis was not supported by the results. The hypothesis was that the ovariectomized ewes and the sham animals would have the same area of secondary bone and number of secondary osteons, including unencroached secondary osteons, but the ovariectomized ewes would also have more encroached secondary osteons and a larger area of encroached secondary bone. This was not the case on either the tensile side or the compressive side. On the tensile side, there was a larger area encroached and more encroached secondary osteons in the OVX animals compared to the sham, but there was also a larger area remodeled and more secondary osteons in the OVX animals compared to the sham. On the compressive side there was no significant difference in the area encroached, amount of encroached secondary osteons per area, area remodeled, or amount of secondary osteons per area between surgery types. However, other conclusions were found from the measurements and analysis which shed light on to different ideas for what is happening in the ovariectomized bone.

The results in the study showed no change in porosity between ovariectomized animals and sham animals, as seen in the results of BV/TV. There was also no difference in porosity from the caudal side compared to the cranial side. This indicates that there is no change in the architecture of the bone between groups. Based on this information, one of two things could have occurred: either nothing happened or a lot happened but in a way that looks like nothing happened because there was no net material loss. If the latter, one explanation could be a change in material due to bone remodeling. Typically when there is an increase in bone remodeling, 
there is also an increase in porosity and a decrease in BV/TV because the Haversian canal porosities created in the center of osteons are larger than the porosities seen in the bone that was replaced [33]. It is possible, however, that the refilling of bone by the osteoblasts when creating osteons replaces the same amount of bone that has been taken away, leading to smaller Haversian canals. Based on results from other measurements other than BV/TV, it seems as though there is some difference in remodeling characteristics between groups even though there is no change in architecture.

When analyzing both types of secondary bone combined, encroached secondary bone and unencroached secondary bone, there is an increase in remodeling on the cranial side with OVX. Both the area remodeled and the number of secondary osteons per area, for the tissue area and material area, were larger in the OVX animals compared to the sham animals. On the caudal side, there was no change in the area or number of secondary osteons with OVX. For both the cranial and caudal side the OVX animals had a significantly larger average area per osteon compared to the sham animals, which could mean than an ovariectomy causes newly formed secondary osteons to be larger than they were before.

Most trends seen with the results of the area remodeled and number of secondary osteons were also seen for the results of the area encroached and number of encroached secondary osteons. There was an increase on the cranial side, with OVX, in the area encroached and amount of encroached osteons, for the tissue and material area. On the caudal side there was no change in encroachment with OVX. However, the average area per osteon was not different between any of the groups, which indicate that the average encroached osteon area does not change. 
The hypothesis also said that the number and area of unencroached secondary osteons would not change due to an ovariectomy. However, the results found that there was a significantly larger area of unencroached secondary bone in animals that had been ovariectomized. Unencroached secondary bone is osteons that are not remodeled away by subsequent generations of osteons, or in other words they are relatively new osteons. When just looking at the results from the fraction unencroached as both tissue and material parameters, one conclusion could be that there is a larger area of new secondary bone as a result of an ovariectomy.

The trend seen in the area of unencroached secondary bone was not the same trend seen in the number of unencroached secondary osteons per tissue and material area. Since the trends are not the same, this indicates that there is a change in osteon size between groups. The trend seen in the amount of unencroached secondary osteons per material and tissue area was that there were fewer unencroached osteons on the caudal side with OVX. There was no change on the cranial side, and the amount of unencroached osteons per material and tissue area on the cranial side was no different than the amount on the caudal side for the sham animals. The results for the area and amount of unencroached osteons indicate that the osteons grown are larger with OVX, and the osteons on the compressive side are generally larger than the osteons on the tensile side.

As determined by previous literature, the anatomical region the bone sample is from can significantly affect the remodeling parameters due to different mechanical environments. In a prior study that used some of the same samples, there was shown to be a significant difference in the area of remodeled bone between different anatomical regions when looking at six anatomical sectors of 12 month sham ovine bone averaged for four different seasons [31]. The anatomical region in the mechanical environment with the largest amount of compression, or the caudal 
region, had the largest area of remodeled bone [31]. The same results were found in 12 month ovine OVX bone samples averaged for four different seasons [32]. Another study found differences in microstructure between opposing compression and tension cortices in horse, elk, and sheep bone samples [34]. The difference in remodeling and microstructure can be explained by Frost's Mechanostat Theory of mechanically induced bone adaption where bone adapts to the needed mechanical properties based on the strain it experiences [12]. Figure 46 visually shows the regional differences in bone microstructure based on the mechanical environment, as seen in the mid shaft of a human femur. These adaptations based on anatomical region help improve the whole bone so that it is best fit for the loads it experiences and reduce fractures. Anatomical differences can be an important factor when determining how an ovariectomy surgery affects ovine bone, rather than just averaging results from all regions of bone analyzed. 

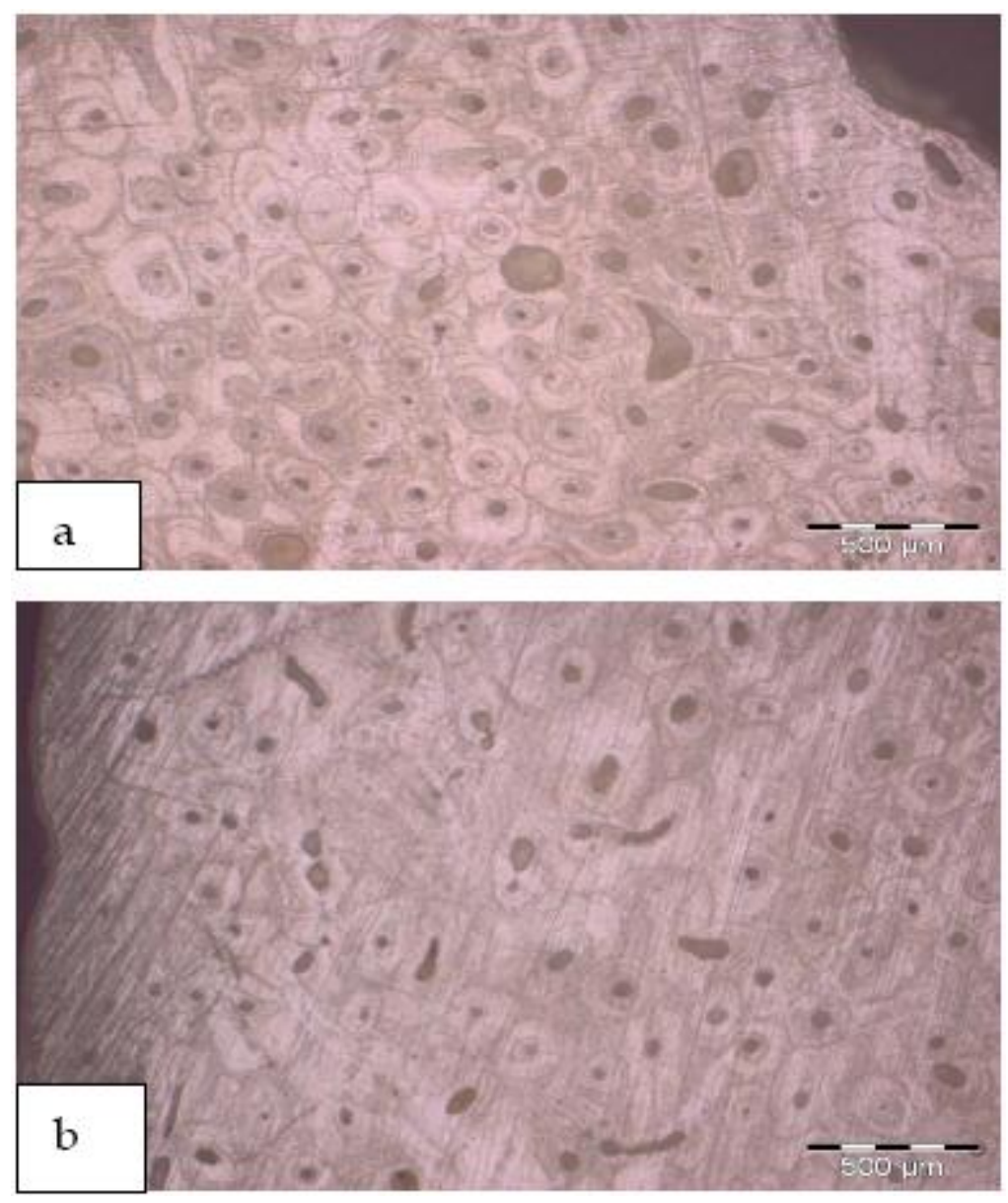

Figure 46. A cross section of cortical bone from the mid shaft of a normal human femur displaying anatomical differences in osteonal density between

(a) the inner side, in compression, and (b) the outer side, in tension [35].

Similar trends were seen in this study for the sham animals; there was more remodeling, both indicated by area remodeled and number of secondary osteons, on the side of bone that was in compression. This can be attributed to bone adaptation when the animal is under normal hormone levels. When the animal is ovariectomized, parts of remodeling begin to change. This main change was seen on the tension side, which showed an increase in remodeling in new places. On the compressive side, the amount remodeling was relatively the same, except that the new osteons grown were larger with the ovariectomy. Because of the increase in remodeling on 
the tensile side, especially that the remodeled area is branching out into new regions, the tensile side is beginning to look like the compressive side. Since the tensile side is acting like the compressive side, normal bone adaptation to the mechanical environment is thrown off. This could lead to diminishing mechanical properties for the structure as a whole because the bone is no longer best suited for the forces it experiences.

Other studies have shown some significant differences between ovariectomized and sham ovine bone in biomechanical properties. One study consisted of two groups of 19 skeletally mature sheep, one group consisting of the sham sheep and the other group consisting of the OVX sheep, where the sheep's left metatarsal underwent unconfined compression testing at the cross section of the mid-diaphysis. The results showed that there was a decrease in stiffness and yield strength in the ovariectomized bones (Figure 47), however, there was no difference in compressive strength between groups [36]. One reason for the decrease in stiffness could be that there is more remodeling as a result of OVX, and new bone is less mineralized resulting in a reduced stiffness. Remodeling is also know to help improve compressive strength, but greatly weaken the tensile strength [37]. Another possible explanation can be made based on the results from this study that showed more remodeling in new places on the tensile side making the tensile side appear more like the compressive side. Since the tensile side is acting like the compressive side, the bone would be stronger in compression, but at the same time weaker in most other mechanical properties, which is what was shown in Kennedy's study. 


\section{A Yield Strength in Control Vs OVX Groups}
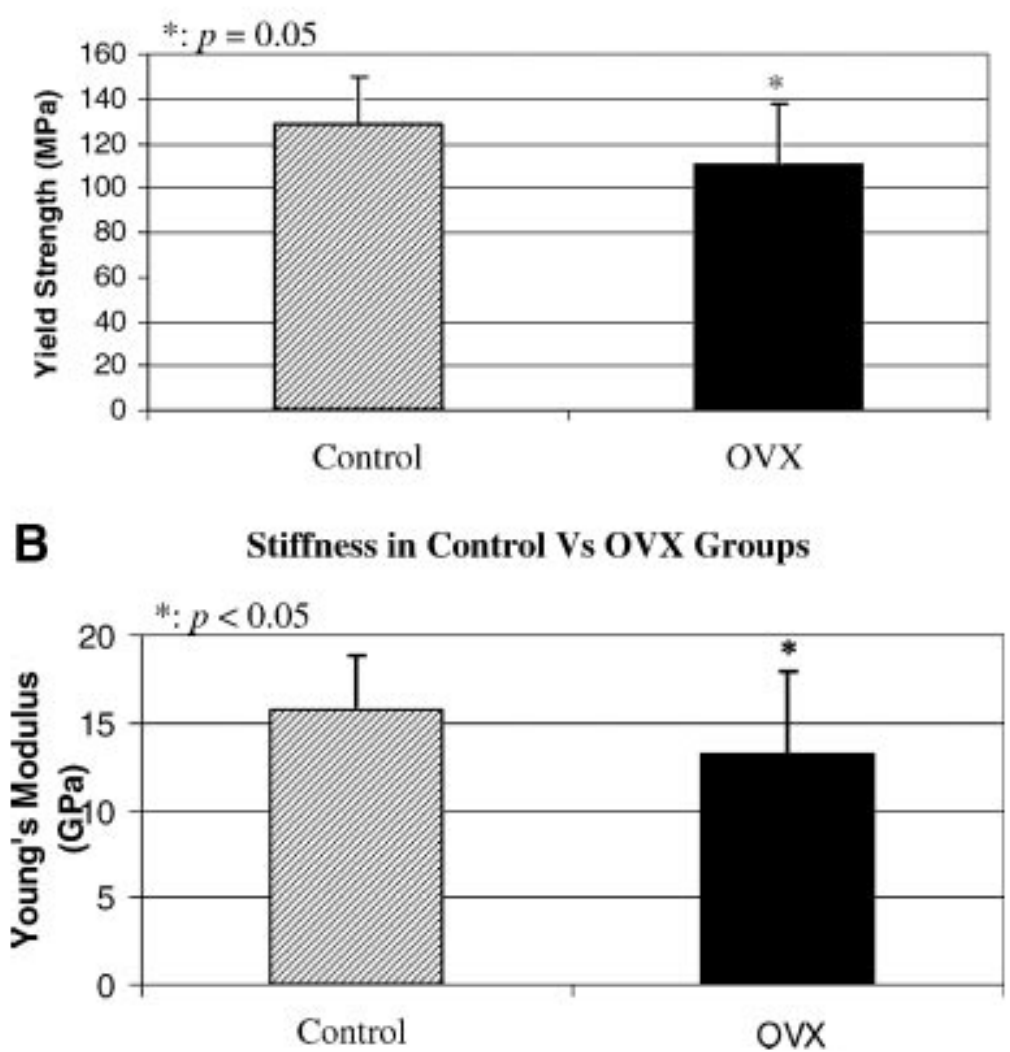

Figure 47. Compact bone yield strength and stiffness for the OVX compared to control groups [36].

Figure 48 displays an illustration of a proposed model for what is occurring in each of the groups in this study. This figure shows the differences between the groups. In the sham animals it shows that the compression side has larger unencroached osteons, a bigger encroached secondary bone area, and more osteons that are encroached. It also shows that the secondary bone on the compressive side is spread out throughout the sample, while the tensile the secondary bone is concentrated in one small area. The samples that are ovariectomized, or the samples with the decreased levels of estrogen, look different than the sham animals. On the tensile side, the ovariectomy causes a bigger encroached and unencroached secondary bone area, larger unencroached secondary osteons, and more encroached secondary osteons. On the 
compressive side, the ovariectomy causes a bigger encroached area compared to the sham animals. For the ovariectomized animals, the samples from the compressive side had larger unencroached osteons while the animals on the tensile side had more osteons that were unencroached.

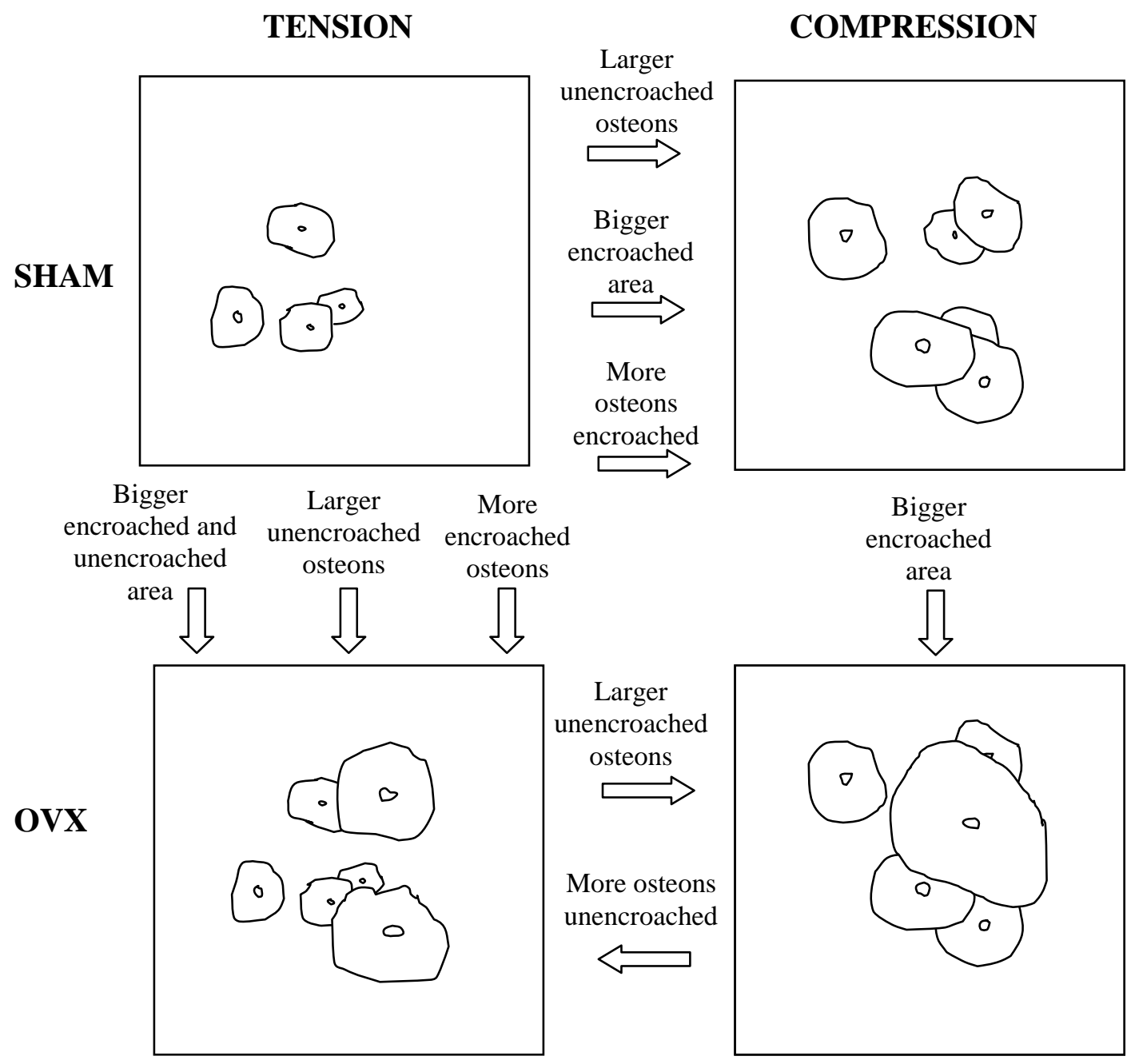

Figure 48. An approximate illustration of how the cumulative area, number, and average size of unencroached and encroached secondary osteons differ between groups based on the effect of sham vs. OVX and compression vs. tension. The encroached secondary osteon average size remained constant in all groups. The arrows indicate a significant increase between groups $(\mathrm{p}<0.05)$. 
Based on these results, a proposed model for what is occurring is that the ovariectomy causes the tensile side to act like the compressive side of the bone. The mechanism for this could be that low levels of estrogen from an ovariectomy surgery lead to increased levels of interleukin 1, interleukin 6, and tumor necrosis factor, as well as decreased levels of transforming growth factor- $\beta$, which can lead to an increased lifetime of osteoclasts. This means that there is an increase in resorption, which can lead to more remodeling. Since there was already remodeling on the compressive side, there was relatively no change. On the tensile side, or a region of the ovine bone that had little remodeling before, there was a dramatic increase in remodeling due to the change in levels of cytokines, making it appear the same as the compressive side. The results showed on the tensile side, there were more new secondary osteons grown at the borders of the previous secondary bone, and also in new locations that used to be just primary bone. On the compressive side, the ovariectomy did not cause too much of a change in remodeling except that the secondary osteons grown were larger than they were before the surgery. The larger osteons could be a result from decreased levels of transforming growth factor, leading to longer lives of osteoclasts, resulting in larger resorption spaces.

The increase in remodeling on the tensile side can explain why the ovariectomized bone is weaker for the yield strength and stiffness tests, while there was no change in the compressive strength. This study showed that decreased levels of estrogen in sheep from an ovariectomy surgery causes weaker bone by regional increases in remodeling. This oncoming homogeneity in the bone could be a sign that the ovariectomy surgery causes a loss of responsiveness of bone to physical loads. 


\subsection{Limitations}

There were a few limitations with this study which should be considered when interpreting the results. One limitation with this study is that the bone tissue samples were made into microradiographs, which are two-dimensional replications, or projections, of threedimensional structures. The different orientations of secondary osteons can change the dimensions to make them seem larger than a perpendicular cross section. The increase in osteon size seen with the ovariectomy surgery may instead indicate a change in osteonal direction rather than larger secondary osteons. Also, since the microradiographs are projections, it can make it difficult to determine if structures are primary bone, unencroached secondary bone, or encroached secondary bone, leading to structures being wrongly identified.

The exposure of the microradiograph also caused problems with making measurements on the samples. Some microradiographs were too dark to analyze, even at the brightest microscope setting. This usually occurred at the edges of specimens resulting in a smaller area to make measurements in. The error due to dark exposure on the edges of specimens was reduced by leaving out quadrants that were too dark from the averages of parameters.

\subsection{Future Work}

Since part of this study was to define a new method to quantify osteon encroachment, the repeatability of this method has only been tested with one individual. These measurements were found to be repeatable within one person making measurements, but they have not yet been determined to be repeatable outside that one person. An inter-observer repeatability study of this method would be a crucial when using this method in future studies to determine the validity of the new measurements. The study could consist of multiple individuals making measurements 
with this method on the same samples, and then statistically analyzing the measurements to determine if there is a significant difference between the individuals.

Previous research on the bone samples the measurements were taken from showed that other factors play a role in the amount of bone remodeling other than just the type of surgery the ewe underwent. The season the sheep underwent surgery played a significant role in the remodeling characteristics of the bone [31]. During the summer months, sheep typically have more remodeling compared to the winter months, since the animals are more active during the summer due to more hours of daylight. Other factors such as seasonal breeding and an annual anestrus period could have also played a role in remodeling characteristics. This study accounted for these affects by only including sheep from the same sacrifice season, although it would be interesting to see if and how osteonal encroachment changes due to seasonal effects. A future study could analyze encroached and unencroached secondary osteons in other seasons that ewes underwent surgery, other than just the summer, to determine if an ovariectomy causes the same trends seen in the summer sheep.

This study only looked at two of the six anatomical sectors which were prepared for histological analysis. It was shown that the two anatomical sectors analyzed, the cranial and caudal sectors, behaved differently from each other when looking at encroached and unencroached secondary bone. In prior studies, the cranial and caudal regions typically had more remodeling than in the other sectors [32]. It would be interesting to analyze encroached and unencroached secondary bone for the four other sectors: the craniomedial, craniolateral, caudomedial, and caudolateral. This would help understand how an ovariectomy affects the whole bone, rather than just two regions. 
All ewes analyzed in this study were sacrificed 12 months after they underwent surgery. The samples used, also had microradiographs for ewes that were sacrificed 3 months after surgery. A future study could analyze the ewes that were sacrificed only 3 months after surgery for encroached and unencroached secondary bone. This data could determine how quickly an ovariectomy can alter the remodeling characteristics by comparing the number of encroached secondary osteons and the area of encroached secondary bone between the sheep of the different sacrifice times.

Another future study to help better understand what is happening to the bone tissue would be to create a computer model that looks at encroachment of osteons. The computer model could be programmed to randomly place secondary osteons in a given area at biological rates that osteons are grown. The amount of encroachment of the secondary osteons could be studied at various time points to determine how much encroachment increases. This would be helpful to determine if at a certain time and when, the amount of encroachment plateaus due to new secondary osteons completely replacing older and encroached secondary osteons. This would also be helpful to determine if the encroached secondary osteons average area changes with different amount of encroachment. Based on the findings of this model, it could support and validate the histological measurements made in this study. 


\section{CONCLUSION}

Determining a new method to measure remodeling by quantifying the encroachment of secondary osteons on previously apposed bone proved to be an informative way to look at cortical bone samples. This method shed a new light on bone remodeling by characterizing secondary bone as either encroached or unencroached, which explained if the secondary bone was continuously remodeled or if the remodeling was a onetime event. This method showed to be repeatable within one individual, but further tests to determine the repeatability outside one individual are needed for this method to be used in the future.

This study composed of two regional samples from the radius of ovine bone that was sacrificed 12 months after either an ovariectomy surgery or a sham surgery. The ovariectomy surgery was conducted to simulate postmenopause in humans due to the lack of estrogen. The results showed significant effects from an ovariectomy that were unique for the tensile and compressive side of the bone. The tensile side showed that an ovariectomy causes bone remodeling to expand its borders and begin remodeling in new locations. The remodeling on the compressive side showed larger new osteons as a result of an ovariectomy, but relatively few changes other than that. The ovariectomy surgery, however, had no effect on the porosity of bone, which contradicts the idea that an increase in porosity is the main result of estrogen loss which is seen in postmenopausal osteoporosis.

Other studies have shown that ovariectomized ewe bone results in weaker mechanical properties of yield strength and stiffness, but no change in compressive strength. These findings combined with the results of this study support the idea that an ovariectomy surgery causes the bone to change material, and not architecture, which is done by an increase in remodeling in regions where there was minimal remodeling before. In other words, the ovariectomy causes the 
tensile side to change its remodeling characteristics to be similar to the compressive side. This alteration in regional bone adaptation makes the bone less suitable for the forces it experiences compared to when the animal had normal levels of estrogen. These findings would be strengthened with a future study that would look at other regional sectors of the radius of the ewe bone. Another future study that would look at the difference in osteonal encroachment less time after surgery would help better understand the mechanism of how remodeling is effected by estrogen depletion. 


\section{REFERENCES}

[1] NIH. NIH Osteoporosis and Related Bone Diseases: National Resource Center. Available from: http://www.bones.nih.gov.

[2] Augat, P., et al., Osteoporosis prevalence and fracture characteristics in elderly female patients with fractures. Archives of Orthopaedic and Trauma Surgery. 130(11): p. 1405-1410.

[3] Poole, K.E.S. and J.E. Compston, Osteoporosis and its management. British Medical Journal, 2006. 333(7581): p. 1251-1256B.

[4] Messinis, I.E., Ovarian feedback, mechanism of action and possible clinical implications. Hum Reprod Update. 2006. 12(5): p. 557-71.

[5] Melton, L.J. How many women have osteoporosis now? J Bone Miner Res, 1995. 10 (2): p 175-7.

[6] Jilka, R.L., Cytokines, bone remodeling, and estrogen deficiency: a 1998 update. Bone, 1998. 23(2): p. 75-81.

[7] Leslie, W.D., S. Morin, and L.M. Lix, A Before-and-After Study of Fracture Risk Reporting and Osteoporosis Treatment Initiation. Annals of Internal Medicine. 153(9): p. 580-W200. 19(12): p. 1233-1240.

[8] Nelson, H.D., et al., Osteoporosis and fractures in postmenopausal women using estrogen. Arch Intern Med, 2002. 162(20): p. 2278-84.

[9] Al-Azzawi, F., Prevention of postmenopausal osteoporosis and associated fractures: Clinical evaluation of the choice between estrogen and bisphosphonates. Gynecol Endocrinol, 2008. 4(11): p. 601-9.

[10] Bone, H.G., et al., Alendronate and estrogen effects in postmenopausal women with low bone mineral density. Alendronate/Estrogen Study Group. J Clin Endocrinol Metab, 2000. 85(2): p. 720-6.

[11] Hazelwood, Scott. Biomedical Engineering 525 Course. California Polytechnic State University, San Luis Obispo. Spring 2012. Lecture.

[12] Martin, R.B., D.B. Burr, and N.A. Sharkey, Skeletal Tissue Mechanics. 2004, New York: Springer.

[13] Ethier, C.R. and C.A. Simmons, Introductory Biomechanics: From Cells to Organisms. 2007, New York: Cambridge University Press.

[14] Saladin, K.S. Anatomy and Physiology: The Unity of Form and Function. 2010, New York: McGraw-Hill Companies, Inc.

[15] Hall, Susan. Basic Biomechanics Fifth Edition. 2007, New York: McGraw-Hill Companies Inc.

[16] Buchman, S.R., et al., Use of microcomputed tomography scanning as a new technique for the evaluation of membranous bone. Journal of Craniofacial Surgery, 1998. 9(1): p. 48-54. 
[17] Recker, R.R., Bone Histomorphometry: Techniques and Interpretation. 1983, Boca Raton: CRC Press.

[18] Hryce, T., Modeling the Long Term Effects of Alendronate on Bone Mass Preservation of the Femur with Articular Surface and Total Hip Replacements, in Biomedical Engineering. Thesis. California Polytechnic State University, 2010. San Luis Obispo: California Polytechnic State University, 2010.

[19] Gibson, V.A., et al., Osteonal effects on elastic modulis and fatigue life in equine bone. Journal of Biomechanics, 2005. 39(2): p. 217- 225.

[20] Kerley, E.R., The microscopic determination of age in human bone. Am J Phys Anthropol, 1965. 23(2): p. 149-164.

[21] Stout, S.D., The application of histomorphometric analysis to ancient skeletal remains. Anthropos (Greece), 1983. 10: p. 60-71.

[22] Burr, D.B., Ruff, C.B., Thompson, D.D., Patterns of skeletal histologic change through time: Comparison of and archic native American population with modern populations. Anat Rec, 1990. 226(3): p. 307-313.

[23] Ericksen, M.F., Histological estimation of age at death using the anterior cortex of the femur. Am J Phys Anthropol, 1991. 84(2): p. 171-179.

[24] Robling, A.G., Stout, S.D., Histomorphometry of the human cortical bone, Applications to age estimation. In Katzenberg, S., Saunders, S., Biological Anthropology of the Human Skeleton, $2^{\text {nd }}$ edition. 2008, New York: Wiley-Liss.

[25] Kubek, D.J., et al., Ovariectomy stimulates and bisphosphonates inhibit intracortical remodeling in the mouse mandible. Orthodontics \& Craniofacial Research, 2010. 13(4): p. 214-222.

[26] Feher, A., et al., Bisphosphonates do not inhibit periosteal bone formation in estrogen deficient animals and allow enhanced bone modeling in response to mechanical loading. Bone, 2010. 46(1): p. 203-207.

[27] Newman, E., et al., The Potential of Sheep for the Study of Osteopenia - Current Status and Comparison with Other Animal-Models. Bone, 1995. 16(4): p. S277-S284.

[28] Baofeng, L., et al., Characterization of a rabbit osteoporosis model induced by ovariectomy and glucocorticoid. Acta Orthop, 2010. 81(3): p. 396-401.

[29] Turner, A.S., The sheep as a model for osteoporosis in humans. Vet J, 2002. 163(3): p. 232-9.

[30] Newton, B.I., et al., The ovariectomized sheep as a model for human bone loss. J Comp Pathol, 2004. 130(4): p. 323-6.

[31] Calcagno, Joseph. Seasonal and Anatomical Variation in Compact Bone Remodeling in the Adult Sheep. Thesis. California Polytechnic State University, 2011. San Luis Obispo: California Polytechnic State University, 2011. 
[32] Wong, Erica. Effects of ovariectomy, seasonal changes, and anatomical position on the compact bone remodeling as seen in the adult ovine model. Thesis. California Polytechnic State University, 2012. San Luis Obispo: California Polytechnic State University, 2012.

[33] Clarke, Bart, Normal Bone Anatomy and Physiology. Clinical Journal of the American Society of Nephrology, 2008. 3(3): p.S131-S139.

[34] Skedros, J.G., Biomechanical implications of mineral content and microstructural variations in cortical bone of horse, elk, and sheep calcanei. Anat Rec, 1997. 249(3): p. 297-316.

[35] Biswas, S., Study of microstructure and mechanical properties of human cortical bone. International Journal of Scientific \& Engineering Research, 2011. 2(3): p. 13-18.

[36] Kennedy, O.D., et al., Effects of ovariectomy on bone turnover, porosity, and biomechanical properties in ovine compact bone 12 months postsurgery. J Orthop Res, 2009. 27(3): p. 303-9.

[37] Currey, John D., Bone Structure and Mechanics. 2002, New Jersey: Princeton University Press. 


\section{Appendix A. Parameter Data}

\begin{tabular}{|c|c|c|c|}
\hline \multicolumn{3}{|c|}{$\begin{array}{c}\text { Average BV/TV } \\
\text { 12 Month Summer Sheep }\end{array}$} \\
\hline Sheep & Surgery & Cranial (Tensile) & Caudal (Compressive) \\
\hline C01 & Control & 0.9351852 & 0.9236111 \\
\hline C03 & Control & 0.9537037 & 0.8958333 \\
\hline C04 & Ovx & 0.9166667 & 0.8472222 \\
\hline C05 & Ovx & 0.9236111 & 0.9305556 \\
\hline C06 & Control & 0.9375 & 0.8888889 \\
\hline C07 & Control & 0.9351852 & 0.9351852 \\
\hline C08 & Ovx & 0.962963 & 0.9259259 \\
\hline C11 & Control & 0.9305556 & 0.9537037 \\
\hline C13 & Ovx & 0.962963 & 0.9444444 \\
\hline C18 & Ovx & 0.8888889 & 0.9791667 \\
\hline C22 & Control & 0.9583333 & 0.9236111 \\
\hline C26 & Control & 0.9652778 & 0.962963 \\
\hline
\end{tabular}

\begin{tabular}{|c|c|c|c|}
\hline \multicolumn{4}{|c|}{$\begin{array}{c}\text { Average Fraction Remodeled (Tissue) } \\
\text { 12 Month Summer Sheep }\end{array}$} \\
\hline Sheep & Surgery & Cranial (Tensile) & Caudal (Compressive) \\
\hline C01 & Control & 0.490741 & 0.430556 \\
\hline C03 & Control & 0.518519 & 0.541667 \\
\hline C04 & Ovx & 0.583333 & 0.375 \\
\hline C05 & Ovx & 0.604167 & 0.611111 \\
\hline C06 & Control & 0.361111 & 0.534722 \\
\hline C07 & Control & 0.351852 & 0.5 \\
\hline C08 & Ovx & 0.564815 & 0.574074 \\
\hline C11 & Control & 0.3125 & 0.472222 \\
\hline C13 & Ovx & 0.601852 & 0.509259 \\
\hline C18 & Ovx & 0.541667 & 0.597222 \\
\hline C22 & Control & 0.479167 & 0.590278 \\
\hline C26 & Control & 0.402778 & 0.601852 \\
\hline
\end{tabular}




\begin{tabular}{|c|c|c|c|}
\hline \multicolumn{4}{|c|}{$\begin{array}{c}\text { Average Fraction Remodeled (Material) } \\
\text { 12 Month Summer Sheep }\end{array}$} \\
\hline Sheep & Surgery & Cranial (Tensile) & Caudal (Compressive) \\
\hline C01 & Control & 0.52475 & 0.46617 \\
\hline C03 & Control & 0.54369 & 0.60465 \\
\hline C04 & Ovx & 0.63636 & 0.44262 \\
\hline C05 & Ovx & 0.65414 & 0.65672 \\
\hline C06 & Control & 0.38519 & 0.60156 \\
\hline C07 & Control & 0.37624 & 0.53465 \\
\hline C08 & Ovx & 0.58654 & 0.62 \\
\hline C11 & Control & 0.33582 & 0.49515 \\
\hline C13 & Ovx & 0.625 & 0.53922 \\
\hline C18 & Ovx & 0.60938 & 0.60993 \\
\hline C22 & Control & 0.5 & 0.6391 \\
\hline C26 & Control & 0.41727 & 0.625 \\
\hline
\end{tabular}

\begin{tabular}{|c|c|c|c|}
\hline \multicolumn{4}{|c|}{ Average Fraction Encroached (Tissue) } \\
12 Month Summer Sheep \\
\hline Sheep & Surgery & Cranial (Tensile) & Caudal (Compressive) \\
\hline C01 & Control & 0.18519 & 0.25 \\
\hline C03 & Control & 0.23148 & 0.20833 \\
\hline C04 & Ovx & 0.29167 & 0.18056 \\
\hline C05 & Ovx & 0.25694 & 0.27778 \\
\hline C06 & Control & 0.09722 & 0.25 \\
\hline C07 & Control & 0.12963 & 0.19444 \\
\hline C08 & Ovx & 0.18519 & 0.22222 \\
\hline C11 & Control & 0.07639 & 0.2037 \\
\hline C13 & Ovx & 0.2037 & 0.22222 \\
\hline C18 & Ovx & 0.23611 & 0.25 \\
\hline C22 & Control & 0.21528 & 0.34028 \\
\hline C26 & Control & 0.16667 & 0.25926 \\
\hline
\end{tabular}




\begin{tabular}{|c|c|c|c|}
\hline \multicolumn{4}{|c|}{ Average Fraction Encroached (Material) } \\
12 Month Summer Sheep \\
\hline Sheep & Surgery & Cranial (Tensile) & Caudal (Compressive) \\
\hline C01 & Control & 0.19802 & 0.27068 \\
\hline C03 & Control & 0.24272 & 0.23256 \\
\hline C04 & Ovx & 0.31818 & 0.21311 \\
\hline C05 & Ovx & 0.2782 & 0.29851 \\
\hline C06 & Control & 0.1037 & 0.28125 \\
\hline C07 & Control & 0.13861 & 0.20792 \\
\hline C08 & Ovx & 0.19231 & 0.24 \\
\hline C11 & Control & 0.08209 & 0.21359 \\
\hline C13 & Ovx & 0.21154 & 0.23529 \\
\hline C18 & Ovx & 0.26563 & 0.25532 \\
\hline C22 & Control & 0.22464 & 0.36842 \\
\hline C26 & Control & 0.17266 & 0.26923 \\
\hline
\end{tabular}

\begin{tabular}{|c|c|c|c|}
\hline \multicolumn{4}{|c|}{ Average Fraction Encroached (Remodeled) } \\
12 Month Summer Sheep \\
\hline Sheep & Surgery & Cranial (Tensile) & Caudal (Compressive) \\
\hline C01 & Control & 0.37736 & 0.58065 \\
\hline C03 & Control & 0.44643 & 0.38462 \\
\hline C04 & Ovx & 0.5 & 0.48148 \\
\hline C05 & Ovx & 0.42529 & 0.45455 \\
\hline C06 & Control & 0.26923 & 0.46753 \\
\hline C07 & Control & 0.36842 & 0.38889 \\
\hline C08 & Ovx & 0.32787 & 0.3871 \\
\hline C11 & Control & 0.24444 & 0.43137 \\
\hline C13 & Ovx & 0.33846 & 0.43636 \\
\hline C18 & Ovx & 0.4359 & 0.4186 \\
\hline C22 & Control & 0.44928 & 0.57647 \\
\hline C26 & Control & 0.41379 & 0.43077 \\
\hline
\end{tabular}




\begin{tabular}{|c|c|c|c|}
\hline \multicolumn{4}{|c|}{ Average Fraction Unencroached (Tissue) } \\
12 Month Summer Sheep \\
\hline Sheep & Surgery & Cranial (Tensile) & Caudal (Compressive) \\
\hline C01 & Control & 0.30556 & 0.18056 \\
\hline C03 & Control & 0.28704 & 0.33333 \\
\hline C04 & Ovx & 0.29167 & 0.19444 \\
\hline C05 & Ovx & 0.34722 & 0.33333 \\
\hline C06 & Control & 0.26389 & 0.28472 \\
\hline C07 & Control & 0.22222 & 0.30556 \\
\hline C08 & Ovx & 0.37963 & 0.35185 \\
\hline C11 & Control & 0.23611 & 0.26852 \\
\hline C13 & Ovx & 0.39815 & 0.28704 \\
\hline C18 & Ovx & 0.30556 & 0.34722 \\
\hline C22 & Control & 0.26389 & 0.25 \\
\hline C26 & Control & 0.23611 & 0.34259 \\
\hline
\end{tabular}

\begin{tabular}{|c|c|c|c|}
\hline \multicolumn{4}{|c|}{ Average Fraction Unencroached (Material) } \\
12 Month Summer Sheep \\
\hline Sheep & Surgery & Cranial (Tensile) & Caudal (Compressive) \\
\hline C01 & Control & 0.32673 & 0.19549 \\
\hline C03 & Control & 0.30097 & 0.37209 \\
\hline C04 & Ovx & 0.31818 & 0.22951 \\
\hline C05 & Ovx & 0.37594 & 0.35821 \\
\hline C06 & Control & 0.28148 & 0.32031 \\
\hline C07 & Control & 0.23762 & 0.32673 \\
\hline C08 & Ovx & 0.39423 & 0.38 \\
\hline C11 & Control & 0.25373 & 0.28155 \\
\hline C13 & Ovx & 0.41346 & 0.30392 \\
\hline C18 & Ovx & 0.34375 & 0.35461 \\
\hline C22 & Control & 0.27536 & 0.27068 \\
\hline C26 & Control & 0.2446 & 0.35577 \\
\hline
\end{tabular}




\begin{tabular}{|c|c|c|c|}
\hline \multicolumn{4}{|c|}{ Average Fraction Unencroached (Remodeled) } \\
12 Month Summer Sheep \\
\hline Sheep & Surgery & Cranial (Tensile) & Caudal (Compressive) \\
\hline C01 & Control & 0.62264 & 0.41935 \\
\hline C03 & Control & 0.55357 & 0.61538 \\
\hline C04 & Ovx & 0.5 & 0.51852 \\
\hline C05 & Ovx & 0.57471 & 0.54545 \\
\hline C06 & Control & 0.73077 & 0.53247 \\
\hline C07 & Control & 0.63158 & 0.61111 \\
\hline C08 & Ovx & 0.67213 & 0.6129 \\
\hline C11 & Control & 0.75556 & 0.56863 \\
\hline C13 & Ovx & 0.66154 & 0.56364 \\
\hline C18 & Ovx & 0.5641 & 0.5814 \\
\hline C22 & Control & 0.55072 & 0.42353 \\
\hline C26 & Control & 0.58621 & 0.56923 \\
\hline
\end{tabular}

\begin{tabular}{|c|c|c|c|}
\hline \multicolumn{4}{|c|}{ Average Secondary Osteons/ Tissue Area } \\
12 Month Summer Sheep \\
\hline Sheep & Surgery & Cranial (Tensile) & Caudal (Compressive) \\
\hline C01 & Control & 31.3333 & 31.25 \\
\hline C03 & Control & 36 & 34.25 \\
\hline C04 & Ovx & 39.5 & 23 \\
\hline C05 & Ovx & 37 & 43 \\
\hline C06 & Control & 30 & 31.75 \\
\hline C07 & Control & 25.6667 & 31.3333 \\
\hline C08 & Ovx & 40 & 34 \\
\hline C11 & Control & 23.25 & 31.3333 \\
\hline C13 & Ovx & 37.3333 & 29.3333 \\
\hline C18 & Ovx & 35.25 & 30.5 \\
\hline C22 & Control & 32 & 46.75 \\
\hline C26 & Control & 32.5 & 33.3333 \\
\hline
\end{tabular}




\begin{tabular}{|c|c|c|c|}
\hline \multicolumn{4}{|c|}{ Average Secondary Osteons/ Material Area } \\
12 Month Summer Sheep \\
\hline Sheep & Surgery & Cranial (Tensile) & Caudal (Compressive) \\
\hline C01 & Control & 33.505 & 33.8346 \\
\hline C03 & Control & 37.7476 & 38.2326 \\
\hline C04 & Ovx & 43.0909 & 27.1475 \\
\hline C05 & Ovx & 40.0602 & 46.209 \\
\hline C06 & Control & 32 & 35.7188 \\
\hline C07 & Control & 27.4455 & 33.505 \\
\hline C08 & Ovx & 41.5385 & 36.72 \\
\hline C11 & Control & 24.9851 & 32.8544 \\
\hline C13 & Ovx & 38.7692 & 31.0588 \\
\hline C18 & Ovx & 39.6563 & 31.1489 \\
\hline C22 & Control & 33.3913 & 50.6165 \\
\hline C26 & Control & 33.6691 & 34.6154 \\
\hline
\end{tabular}

\begin{tabular}{|c|c|c|c|}
\hline \multicolumn{4}{|c|}{ Average Encroached Secondary Osteons/ Tissue Area } \\
12 Month Summer Sheep \\
\hline Sheep & Surgery & Cranial (Tensile) & Caudal (Compressive) \\
\hline C01 & Control & 12.6667 & 16.25 \\
\hline C03 & Control & 16 & 15 \\
\hline C04 & Ovx & 22.5 & 12.5 \\
\hline C05 & Ovx & 17 & 21 \\
\hline C06 & Control & 10.25 & 15.5 \\
\hline C07 & Control & 10.3333 & 13.6667 \\
\hline C08 & Ovx & 18.3333 & 16.3333 \\
\hline C11 & Control & 7 & 13.3333 \\
\hline C13 & Ovx & 15.6667 & 14 \\
\hline C18 & Ovx & 15.5 & 14 \\
\hline C22 & Control & 15.5 & 25 \\
\hline C26 & Control & 13 & 15.3333 \\
\hline
\end{tabular}




\begin{tabular}{|c|c|c|c|}
\hline \multicolumn{4}{|c|}{ Average Encroached Secondary Osteons/ Material Area } \\
12 Month Summer Sheep \\
\hline Sheep & Surgery & Cranial (Tensile) & Caudal (Compressive) \\
\hline C01 & Control & 13.5446 & 17.594 \\
\hline C03 & Control & 16.7767 & 16.7442 \\
\hline C04 & Ovx & 24.5455 & 14.7541 \\
\hline C05 & Ovx & 18.406 & 22.5672 \\
\hline C06 & Control & 10.9333 & 17.4375 \\
\hline C07 & Control & 11.0495 & 14.6139 \\
\hline C08 & Ovx & 19.0385 & 17.64 \\
\hline C11 & Control & 7.52239 & 13.9806 \\
\hline C13 & Ovx & 16.2692 & 14.8235 \\
\hline C18 & Ovx & 17.4375 & 14.2979 \\
\hline C22 & Control & 16.1739 & 27.0677 \\
\hline C26 & Control & 13.4676 & 15.9231 \\
\hline
\end{tabular}

\begin{tabular}{|c|c|c|c|}
\hline \multicolumn{4}{|c|}{ Average Unencroached Secondary Osteons/ Tissue Area } \\
12 Month Summer Sheep \\
\hline Sheep & Surgery & Cranial (Tensile) & Caudal (Compressive) \\
\hline C01 & Control & 18.6667 & 15 \\
\hline C03 & Control & 20 & 19.25 \\
\hline C04 & Ovx & 17 & 10.5 \\
\hline C05 & Ovx & 20 & 22 \\
\hline C06 & Control & 19.75 & 16.25 \\
\hline C07 & Control & 15.3333 & 17.6667 \\
\hline C08 & Ovx & 21.6667 & 17.6667 \\
\hline C11 & Control & 16.25 & 18 \\
\hline C13 & Ovx & 21.6667 & 15.3333 \\
\hline C18 & Ovx & 19.75 & 16.5 \\
\hline C22 & Control & 16.5 & 21.75 \\
\hline C26 & Control & 19.5 & 18 \\
\hline
\end{tabular}




\begin{tabular}{|c|c|c|c|}
\hline \multicolumn{4}{|c|}{ Average Unencroached Secondary Osteons/ Material Area } \\
12 Month Summer Sheep \\
\hline Sheep & Surgery & Cranial (Tensile) & Caudal (Compressive) \\
\hline C01 & Control & 19.9604 & 16.2406 \\
\hline C03 & Control & 20.9709 & 21.4884 \\
\hline C04 & Ovx & 18.5455 & 12.3934 \\
\hline C05 & Ovx & 21.6541 & 23.6418 \\
\hline C06 & Control & 21.0667 & 18.2813 \\
\hline C07 & Control & 16.396 & 18.8911 \\
\hline C08 & Ovx & 22.5 & 19.08 \\
\hline C11 & Control & 17.4627 & 18.8738 \\
\hline C13 & Ovx & 22.5 & 16.2353 \\
\hline C18 & Ovx & 22.2188 & 16.8511 \\
\hline C22 & Control & 17.2174 & 23.5489 \\
\hline C26 & Control & 20.2014 & 18.6923 \\
\hline
\end{tabular}

\begin{tabular}{|c|c|c|c|}
\hline \multicolumn{4}{|c|}{$\begin{array}{c}\text { Average Secondary Osteon Average Area } \\
\text { 12 Month Summer Sheep } \\
\text { (osteons/mm }{ }^{2} \text { ) }\end{array}$} \\
\hline Sheep & Surgery & Cranial (Tensile) & Caudal (Compressive) \\
\hline C01 & Control & 0.01566 & 0.01378 \\
\hline C03 & Control & 0.0144 & 0.01582 \\
\hline C04 & Ovx & 0.01477 & 0.0163 \\
\hline C05 & Ovx & 0.01633 & 0.01421 \\
\hline C06 & Control & 0.01204 & 0.01684 \\
\hline C07 & Control & 0.01371 & 0.01596 \\
\hline C08 & Ovx & 0.01412 & 0.01688 \\
\hline C11 & Control & 0.01344 & 0.01507 \\
\hline C13 & Ovx & 0.01612 & 0.01736 \\
\hline C18 & Ovx & 0.01537 & 0.01958 \\
\hline C22 & Control & 0.01497 & 0.01263 \\
\hline C26 & Control & 0.01239 & 0.01806 \\
\hline
\end{tabular}




\begin{tabular}{|c|c|c|c|}
\hline \multicolumn{4}{|c|}{$\begin{array}{c}\text { Average Encroached Secondary Osteon Average Area } \\
\text { 12 } \\
\text { Month Summer Sheep } \\
\text { (osteons/mm } \text { ( }^{\text {) }}\end{array}$} \\
\hline Sheep & Surgery & Cranial (Tensile) & Caudal (Compressive) \\
\hline C01 & Control & 0.03874 & 0.0265 \\
\hline C03 & Control & 0.03241 & 0.03611 \\
\hline C04 & Ovx & 0.02593 & 0.03 \\
\hline C05 & Ovx & 0.03554 & 0.0291 \\
\hline C06 & Control & 0.03523 & 0.0345 \\
\hline C07 & Control & 0.03405 & 0.03659 \\
\hline C08 & Ovx & 0.03081 & 0.03515 \\
\hline C11 & Control & 0.04464 & 0.03542 \\
\hline C13 & Ovx & 0.03842 & 0.03638 \\
\hline C18 & Ovx & 0.03495 & 0.04266 \\
\hline C22 & Control & 0.03091 & 0.02361 \\
\hline C26 & Control & 0.03098 & 0.03925 \\
\hline
\end{tabular}

\begin{tabular}{|c|c|c|c|}
\hline \multicolumn{4}{|c|}{$\begin{array}{c}\text { Average Unencroached Secondary Osteon Average Area } \\
12 \text { Month Summer Sheep } \\
\left(\text { osteons } / \mathrm{mm}^{2}\right)\end{array}$} \\
\hline Sheep & Surgery & Cranial (Tensile) & Caudal (Compressive) \\
\hline $\mathrm{C} 01$ & Control & 0.02629 & 0.0287 \\
\hline $\mathrm{C} 03$ & Control & 0.02593 & 0.02814 \\
\hline $\mathrm{C} 04$ & Ovx & 0.03431 & 0.03571 \\
\hline $\mathrm{C} 05$ & Ovx & 0.03021 & 0.02778 \\
\hline $\mathrm{C} 06$ & Control & 0.01828 & 0.03291 \\
\hline $\mathrm{C} 07$ & Control & 0.02295 & 0.0283 \\
\hline $\mathrm{C} 08$ & Ovx & 0.02607 & 0.03249 \\
\hline C11 & Control & 0.01923 & 0.02623 \\
\hline C13 & Ovx & 0.02778 & 0.03321 \\
\hline C18 & Ovx & 0.02743 & 0.0362 \\
\hline $\mathrm{C} 22$ & Control & 0.02904 & 0.02714 \\
\hline $\mathrm{C} 26$ & Control & 0.02066 & 0.03344 \\
\hline
\end{tabular}

